\title{
Mesons in gauge/gravity duals
}

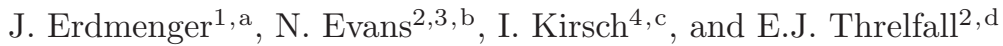 \\ 1 Max-Planck-Institut für Physik (Werner-Heisenberg-Institut), Föhringer Ring 6, 80805 München, Germany \\ 2 School of Physics and Astronomy, Southampton University, Southampton, S017 1BJ, UK \\ 3 Isaac Newton Institute for Mathematical Sciences, 20 Clarkson Road, Cambridge, CB3 0EH, UK \\ 4 Institut für Theoretische Physik, ETH Zürich, CH-8093 Zürich, Switzerland
}

Received: 14 December 2007

Published online: 27 February 2008 - (C) Società Italiana di Fisica / Springer-Verlag 2008 Communicated by A. Schäfer

\begin{abstract}
We review recent progress in studying mesons within gauge/gravity duality, in the context of adding flavour degrees of freedom to generalizations of the AdS/CFT correspondence. Our main focus is on the "top-down approach" of considering models constructed within string theory. We explain the stringtheoretical constructions in detail, aiming at non-specialists. These give rise to a new way of describing strongly coupled confining large- $N$ gauge theories similar to large- $N$ QCD. In particular, we consider gravity dual descriptions of spontaneous chiral symmetry breaking, and compare with lattice results. A further topic covered is the behaviour of flavour bound states in finite-temperature field theories dual to a gravity background involving a black hole. We also describe the "bottom up" phenomenological approach to mesons within AdS/QCD. Some previously unpublished results are also included.
\end{abstract}

PACS. 11.15.-q Gauge field theories - 11.25.Tq Gauge/string duality - 12.40.Yx Hadron mass models and calculations - 14.40.-n Mesons

\section{Contents}

1 Introduction . . . . . . . . . . . 82

2 Brief introduction to the AdS/CFT correspondence. 84

2.1 The basics of string theory . . . . . . . . . 84

2.1.1 D branes . . . . . . . . . . . 85

$2.2 \mathcal{N}=4$ super-Yang-Mills theory . . . . . . . 86

2.3 AdS/CFT correspondence . . . . . . . . . . 86

2.4 Holographic RG flows . . . . . . . . . . . 87

2.5 Confinement. . . . . . . . . . . 88

2.5.1 Heavy-source interaction energy . . . . 88

2.5.2 A discrete glueball spectrum . . . . . . 89

3 AdS/CFT with flavour . . . . . . . . . . . 89

3.1 The D3/D7 brane intersection . . . . . . . . . 90

3.1.1 Field theory of the D3/D7 brane intersection ................. 90 90

3.2 The probe brane correspondence . . . . . . . . 91

3.3 D7 brane fluctuations and mesons in $\mathcal{N}=2$ theory 92 3.3.1 Scalar field fluctuations (spin 0) . . . 92

3.3.2 Fermionic fluctuations ( $\left.\operatorname{spin} \frac{1}{2}\right) \ldots \ldots 9$

3.3.3 Gauge field fluctuations (spin 1) . . . . 94

3.3.4 Fluctuation-operator matching . . . . . 94

3.3.5 Interactions . . . . . . . . . . . . . . 95

\footnotetext{
a e-mail: jke@mppmu.mpg.de

b e-mail: evans@phys.soton.ac.uk

c e-mail: kirsch@phys.ethz.ch

d e-mail: ejt@phys.soton.ac.uk
}

3.3.6 Mesons on the Coulomb branch . . . . . 95

3.4 Holographic heavy-light mesons . . . . . . . . 96

3.4.1 Semi-classical action for heavy-light states 97

3.4.2 Heavy-light mesons from non-Abelian

DBI action . . . . . . . . . . . 9 97

3.5 Mesons with large spin $(J \gg 1) \ldots . . . . .100$

3.6 The squark sector from instantons on the D7 probe ....................... 101

3.7 Summary . . . . . . . . . . . . . . . . . . 103

4 Beyond the probe approximation (backreaction) . . 103

4.1 Fully localized D3/D7 brane intersection . . . . 103

4.1.1 The $\mathcal{N}=2$ field theory at finite $N_{f} / N .103$

4.1.2 The D3/D7 supergravity solution . . . . 104

4.1.3 Non-perturbative completion and $U(1)_{\mathcal{R}}$ chiral anomaly ............ . . 105

4.1.4 Meson computation ... . . . . . 106

5 More supersymmetric mesons . . . . . . . . . . . . 106

5.1 Klebanov's duals . . . . . . . . . . . 106

$5.2 B$ fields in the background: Polchinski-Strassler dual . . . . . . . . . . . . 106

5.3 Maldacena-Nuñez dual . . . . . . . . . . . . . 107

5.4 Defect theories . . . . . . . . . . . 107

5.5 Non-commutativity . . . . . . . . . . . . . 107

6 Chiral symmetry breaking . . . . . . . . . . . . 107

6.1 Chiral symmetry breaking in field theory . . . . 107

6.2 D7 probes in non-supersymmetric backgrounds 108

6.2.1 Constable-Myers background . . . . . . 108

6.2.2 Goldstone boson . . . . . . . . . . . . . 111 
6.2 .3 Vector mesons . . . . . . . . . . . 111

6.3 Gauge theory in $A d S_{4}$ space . . . . . . . . . . . 112

6.4 Chiral symmetry breaking in the D4/D6 system 112 6.4.1 D4 brane background . . . . . . . . . 113

6.4.2 Probe D6 branes . . . . . . . . . . . . . 113

6.5 Non-Abelian chiral symmetries . . . . . . . . . 114

6.5.1 Gravitational background (D4-D8- $\overline{\mathrm{D} 8}$ ) . 114

6.5.2 Probe D8 branes . . . . . . . . . . . . . 115

6.5.3 The pion ............. 116

6.5.4 Meson spectrum and interactions . . . . 116

6.5.5 Non-anti-podal embeddings . . . . . . . 117

6.6 More chiral symmetry breaking . . . . . . . . . 117

6.7 Summary . . . . . . . . . . . . . . . . 117

7 Mesons at finite temperature . . . . . . . . . . 118

7.1 First-order phase transition in the quark-gluon

plasma . . . . . . . . . . . . . 118

7.1.1 AdS-Schwarzschild solution . . . . . . 118

7.1.2 Embedding of a D7 brane . . . . . . . . 118

7.1.3 First-order phase transition at finite temperature . . . . . . . . . 119

7.2 Mesons in the AdS black-hole background . . . 120

7.3 More thermodynamics . . . . . . . . . . . . 122

7.4 Mesons from D7 branes with external $B$ fields . 123

7.5 Summary . . . . . . . . . . . . . . . . 123

8 AdS/QCD . . . . . . . . . . . . . . . . . . . 124

8.1 A simple model . . . . . . . . . . . . . . . 124

8.2 Higher-order pion interactions . . . . . . . . . . . 125

8.3 Glueballs . . . . . . . . . . . . . . . . 125

8.4 A plethora of AdS/QCD phenomenology . . . . 125

8.5 Regge behaviour and the soft wall . . . . . . 126

8.6 Improvement and perfection . . . . . . . . . . 126

8.7 Summary . . . . . . . . . . . . . . . . 126

9 Conclusion . . . . . . . . . . . . . . . . 127

10 Other reviews . . . . . . . . . . . . . . . 127

\section{Introduction}

String theory ${ }^{1}$ originated as a theory of hadrons in the 1960s, when it was noticed that the hadron spectra contain Regge trajectories that can be reproduced by the properties of a rotating relativistic string. However, it was subsequently realized that four-dimensional string theories contain unphysical modes such as tachyons and a massless vector particle. At that time string theory was abandoned as a theory of the strong interactions, and took a rather different route as a promising candidate for a unified theory of all four fundamental interactions including gravity, due to the fact that it contains a graviton in its spectrum. It was realized that a fully consistent string theory must contain supersymmetry and live in ten space-time dimensions. Frustratingly, since gravity is so weak, none of the novel physics of string theory need appear experimentally below energies close to the Planck scale $\left(10^{19} \mathrm{GeV}\right)$, making the ideas of string theory difficult to test.

Since the 1970s though, our understanding of the strong interactions has developed greatly. Quantum Chromodynamics (QCD) has established itself as a very successful quantum field theory description of the strong in-

1 Some introductory texts on string theory are listed in [1-5]. teractions, and is by now very well tested experimentally. The matter degrees of freedom in QCD consist of quarks transforming in the fundamental representation of a non-Abelian $S U(3)$ gauge theory. Interactions are mediated by gauge bosons, the gluon fields, in the adjoint of $S U(3)$. The theory has been shown to be asymptotically free $[6,7]$. This means that at arbitrarily large energy scales, or equivalently at very short distances, the quarks become weakly interacting, whilst at long distances the force becomes ferociously strong. The result of the stronginteraction regime is that quarks are confined into bound states, the hadrons. In addition, the dynamics generate a large constituent mass for the quarks which mixes leftand right-handed quarks, and hence breaks their chiral symmetries.

QCD does provide a heuristic understanding for why the hadron spectrum looks like a string spectrum. An excited meson may be thought of as a quark and an antiquark connected by a tube of strong-interaction flux. Such a configuration indeed resembles a string. 't Hooft made an additional step towards making the connection more concrete when he noticed that $S U(N)$ gauge theories with a large number of colours $N$ simplify [8]. The leading Feynman diagrams in an expansion in $N$ are planar diagrams. The description of a meson in this limit has two quark lines propagating in time connected by a dense "sheet" of gluons - it suggests the worldsheet swept out by a string through time. An explicit understanding of the relation remained mysterious though.

Despite the successes of both string theory and QCD, a number of unsolved issues remain in both areas. On the one hand, it would be desirable to find closer links between string theory and experimentally testable theories. On the other hand, there are properties of QCD which are still poorly understood. Despite the tremendous successes of large-scale computer simulations (lattice gauge theory - for introductory texts see [9-11]), in particular, the lowenergy mechanisms in QCD for confinement and chiral symmetry breaking remain unclear conceptually. New theoretical input in addition to lattice gauge theory appears to be desirable. String theory always seemed like a potential candidate to provide new insights.

These questions have recently led to new relations between modern superstring theory and QCD. These new relations have been made possible by the second superstring revolution in 1995, introducing the concept of $D$ branes [12-14]. D branes arose, on the one hand, as solitonic solutions of ten-dimensional supergravity (the low-energy effective theory of superstring theory at scales lower than the string scale), and, on the other hand, as hypersurfaces in the fundamental string theory on which open strings can end. In the first of these pictures, the excitations are gravitational closed-string modes sourced by the tension of the brane. In the second, where the charged endpoints of open strings move on the D branes, the lowenergy limit of the lightest string is a gauge theory.

This "dual" interpretation of D branes is at the heart of the AdS/CFT correspondence (AdS: anti-deSitter space, CFT: conformal field theory) put forward 
by Maldacena in 1997 [15]. In its original form, this correspondence provides a map between a highly symmetric, strongly coupled large- $N$ gauge theory and a weakly coupled supergravity theory. The gauge theory is just the simplest $(3+1)$-dimensional theory to emerge on the worldvolume of the most basic D3 brane configuration. A number of $N$ coincident D3 branes generates an $S U(N)$ gauge theory in the low-energy limit. $N$ must be large since very many D3 branes are required in order to ensure that the dual supergravity background is weakly coupled. Gauge invariant composite operators of the quantum field theory are mapped to supergravity fields in the same representation of the large symmetry group present. For this original case, many non-trivial tests have been found. The field theory is $\mathcal{N}=4$ large- $N S U(N)$ gauge theory (in addition to the usual gauge fields there are 4 two-component gauginos and 6 real adjoint scalars), whose $\beta$-function has been shown to vanish to all orders in perturbation theory, and thus is conformal even when quantized. More precisely, the correlation functions of the quantum field theory -which, since they involve expectation values, are classical functions although involving Hilbert space operators - are mapped to classical correlation functions in supergravity.

An obvious question after the discovery of this duality was whether it could teach us about QCD, a different strongly coupled gauge theory. To achieve a description of QCD-like theories, it is necessary to break supersymmetry and to remove conformal invariance, so as to obtain a running coupling, as well as to introduce quark fields. Technologies have been developed that allow all of these required features at least to some degree. This review will discuss these technologies, their implications for QCD and their limitations. A main feature is that so far, gauge/gravity dualities describe large- $N$ field theories only.

It was suggested very shortly after Maldacena's original paper to find gravity duals of less symmetric large- $N$ gauge theories, in particular of confining theories. A number of examples of gravity duals of quantum field theories with less supersymmetry and running couplings have been found. Examples include renormalization group flows obtained by adding relevant operators, for instance, mass terms for the adjoint fermions and scalars present in $\mathcal{N}=4$ theory. These perturbations can be chosen to maintain some or none of the supersymmetries of the original model. The common feature is that a strongly coupled gauge theory is mapped to a weakly coupled -i.e. solvable - classical gravity theory. Whilst this is considerable progress, it must be noted that the relevant operators are essentially perturbations - since the gauge dynamics is strongly coupled at all energy scales, one cannot completely decouple massive fields from the dynamics. The far ultraviolet region of these theories generically displays a large degree of supersymmetry. On the other hand, a mass perturbation in a conformal field theory fundamentally changes the dynamics, and the resulting behaviour of these theories is very different from the conformal $\mathcal{N}=4$ theory.
Further progress has been made by adding flavour degrees of freedom in the fundamental representation of the gauge group to the gravity dual description. The original Maldacena set-up contains $N(3+1)$-dimensional D3 branes, on which open strings, which have charged endpoints, may end. This corresponds to $\mathcal{N}=4 S U(N)$ gauge theory which has only adjoint degrees of freedom. The addition of different types of branes into the set-up introduces strings stretched between the new brane and the D3 branes - these strings have only one charge under the $S U(N)$ group on the D3 branes and are therefore quark fields. The best understood example consists of a small number, $N_{f}$, of $(7+1)$-dimensional D7 probe branes [16]. Treating them as a probe means they do not change the background geometry or, in the gauge theory language, that quark loop effects are suppressed in the gauge background - this corresponds to the quenched approximation which is formally valid when $N_{f} \ll N^{2}$. In the supergravity picture, one has $A d S_{5} \times S^{5}$ generated by the D3 branes with the D7 brane probe wrapping - for massless quarks - an $A d S_{5} \times S^{3}$ subspace. This corresponds to a four-dimensional $\mathcal{N}=2$ supersymmetric large- $N$ gauge theory with the field content of $\mathcal{N}=4$ plus a small number of fundamental hypermultiplets. It is the remaining supersymmetry of this theory that makes a clear analysis possible.

Strings with both ends on the flavour brane are dual to quark-antiquark operators (they are in the adjoint of $S U\left(N_{f}\right)$ ). On the gravity side of the correspondence, these strings describe the vacuum position of the brane and its fluctuations if perturbed. The embedding of a brane in a geometry dual to a gauge background therefore encodes the mass and quark bilinear condensate in the theory. Linearized fluctuations are dual to mesonic excitations in the gauge theory. It is possible to extract the bound-state masses [17]. In the supersymmetric theory of D3 and D7 branes, supersymmetry forbids a quark condensate. The meson spectrum consists of tightly bound states of a quark and its antiquark - the mass of the bound state is smaller than the mass of the constituent quarks' mass by a factor of the square root of the 't Hooft coupling $\lambda \equiv g_{Y M}^{2} N$. On the other hand, mesons made of two quarks with distinct masses are heavier with mass of order the heavier quark mass. The suppression of some meson masses relative to others is rather different from what is observed in QCD. This suppression is a result of the very strong coupling present in the models across a large range of energy scales.

The next step towards QCD is to combine supersymmetry breaking deformations of the original $A d S_{5} \times S^{5}$ background and the adding of D7 brane probes to include quarks. In the UV, the field theory returns to the fourdimensional $\mathcal{N}=2$ theory of [16], but the IR is QCDlike with a running gauge coupling. This combination has

\footnotetext{
${ }^{2}$ However, the effect of quark degrees of freedom on flavour physics may indeed be described in the gauge/gravity dual approximation. The prime example for this is the study of the condensate phase diagram in the presence of a quark chemical potential.
} 
been used to obtain a gravity dual description of dynamical chiral symmetry breaking by a quark condensate [18]. Moreover, the associated Goldstone boson has been identified: It is obtained from the fluctuations of the probe D7 brane around its minimum energy configuration. Since in this set-up the spontaneously broken symmetry is $U(1)_{A}$, which is non-anomalous in the limit $N \rightarrow \infty$, the Goldstone boson corresponds to the $\eta^{\prime}$. The $\rho$ mass, as well as interaction terms involving both the Goldstone field and the $\rho$, can also be computed. Comparison with recent lattice results $[19,20]$ for $m_{\rho}$ and $m_{\pi}$ at large $N$ is possible and shows good agreement, at least for small quark mass.

Several similar scenarios in which supersymmetry breaking leads to chiral symmetry breaking have also been found - for example a set-up of D4 and D6 branes [21], by placing the gauge theory on an anti-de-Sitter space [22], or introducing a background magnetic field [23].

More recently a string theory model of D4, D8 and $\overline{\mathrm{D} 8}$ branes has been constructed in $[24,25]$ which realizes the larger non-Abelian $S U\left(N_{f}\right) \times S U\left(N_{f}\right)$ chiral symmetry of QCD and its spontaneous breaking to $S U\left(N_{f}\right)$. The symmetry is broken when the $\mathrm{D} 8$ and $\overline{\mathrm{D} 8}$ brane probes join to form a continuous object. In this approach, meson masses such as for instance of the $\rho$ and $a_{1}$ have been calculated, with results surprisingly close to experimental measurements. However, as in the D4/D6 case, in the far UV the corresponding gauge theory runs to a five-dimensional non-renormalizable theory.

These string theory models have inspired phenomenological approaches to QCD dubbed AdS/QCD. AdS/QCD are a group of models that are essentially a distillation of the key elements of the string models above relevant to QCD phenomenology. Parameters such as the 't Hooft coupling and the quark mass are fitted to the QCD data, and predictions result for the meson masses and couplings. The agreement is surprisingly good (typically of order $10 \%$ ) although systematic errors are uncalculable. Again one should stress that one would expect the results to suffer from being at large $N$, from near-conformality, from the presence of (broken) superpartners and from the quenched approximation.

Some progress has been made towards addressing the quenching issue. In the supersymmetric D3/D7 model, it is possible to investigate also the case of a large number of flavours, of the same order as the number of colours [26-30]. In this case, even in the presence of supersymmetry the beta function is no longer zero, and there is a Landau pole in the UV. On the gravity side, the backreaction has to be taken into account, and the gravity dual of the Landau pole is identified as a certain singularity.

A very fruitful area for extended gauge/gravity dualities is the case of finite-temperature field theories, whose gravity dual is given by a black-hole background [31,32]. In this case, gauge/gravity duality is ideally suited for describing dynamical and non-equilibrium processes. This is considered to be of particular importance for the physics of the quark-gluon plasma as studied at the RHIC accelerator. At high temperature or density, mesons become unstable and melt into the quark-gluon plasma. This phe- nomenon is obtained in the gauge/gravity dual description, but is also associated with a particular first-order phase transition $[18,33-36]$ which is not expected to be present in QCD.

We thus see that the string theory gravity dual picture of strongly coupled gauge theory is beginning to make contact with QCD physics. Qualitatively, the pictures are beginning to match well and in some cases quantitative predictions are not widely off the mark. The possibilities for this technology appear promising. In this review we will develop each of these subjects pedagogically for the interested but non-specialist reader.

This review is organized as follows. We begin in sect. 2 with a brief description of the AdS/CFT correspondence, including a short summary of string theory which serves as a reference in subsequent sections. In sect. 3 we describe in detail how flavour degrees of freedom, i.e. quarks, may be added to the AdS/CFT correspondence, keeping the number of flavours $N_{f}$ much smaller than the number of colours $N \rightarrow \infty$. In sect. 4 we move beyond this limit, the so-called probe limit, and consider the case that $N_{f} \sim N$. In sect. 5 we describe mesons in further supersymmetric geometries. In sect. 6 we consider in detail how chiral symmetry breaking arises in non-supersymmetric geometries. Section 7 is devoted to the gravity dual description of field theories at finite temperature. In sect. 8, the phenomenological AdS/QCD approach is presented, also referred to as "bottom-up" approach. We briefly conclude in sect. 9 with general comments.

For readers unfamiliar with the subject, we recommend reading the following sections first (in the order given): 2 , $3.1,3.2,3.3 .1,6,7$ and 8 .

\section{Brief introduction to the AdS/CFT correspondence}

\subsection{The basics of string theory}

String theory [1-5] plays a major role in the holographic approach to mesons in strongly coupled gauge theories as described in this review, so we here provide a very brief overview to remind the reader and set conventions.

The action of a relativistic string is given by the area of the worldsheet it sweeps out in time written in NambuGoto form as

$$
S=T \int \mathrm{d} \tau \mathrm{d} \sigma \sqrt{\operatorname{det} P\left[G_{a b}\right]}, \quad P\left[G_{a b}\right]=G_{\mu \nu} \frac{\mathrm{d} X^{\mu}}{\mathrm{d} \sigma^{a}} \frac{\mathrm{d} X^{\nu}}{\mathrm{d} \sigma^{b}} .
$$

Here $T \equiv 1 / 2 \pi \alpha^{\prime}$ is the string tension; $\sigma^{a}=(\tau, \sigma)$ are the time and space coordinates on the worldsheet; $P$ represents the "pullback" of the metric as shown; and $G_{\mu \nu}$ is the background metric.

The action can be recast in Polyakov form by introducing a worldsheet metric $h_{a b}$. The action is then

$$
S=-\frac{1}{4 \pi \alpha^{\prime}} \int \mathrm{d}^{2} \sigma \sqrt{-h} h^{a b} \partial_{a} X^{\mu} \partial_{b} X^{\nu} G_{\mu \nu},
$$


but there is also a constraint

$$
T_{a b}=\partial_{a} X^{\mu} \partial_{b} X_{\mu}-\frac{1}{2} h_{a b} h^{c d} \partial_{c} X^{\mu} \partial_{d} X_{\mu}=0 .
$$

There is sufficient symmetry such that the worldsheet metric can be made flat, $h^{a b}=\eta^{a b}$, by a conformal transformation (or more precisely by a Weyl transformation and reparametrization of the worldsheet coordinates).

Classically, the unexcited string is massless with excitations of oscillations on the string's surface forming a tower of states with masses in units of $\sqrt{T}$. The zero point energies of these oscillations contribute a constant negative shift of this spectrum in the quantum theory. The only known way to remove the tachyonic modes is to impose supersymmetry. For this purpose, a worldsheet, twocomponent real fermion is added to the action. Moreover, in space-time, the GSO projection must be imposed to remove states, leaving a supersymmetric space-time theory.

The worldsheet conformal invariance $\left(h_{a b} \rightarrow e^{\phi} h_{a b}\right)$ is anomalous in the quantum theory, unless the theory lives in 10 space-time dimensions.

Oscillations of open strings give rise to massless gauge multiplets (multiple charges are included via Chan-Paton factors, global charges, attached to the ends of the strings, such that non-Abelian gauge symmetries may be realized). Closed string loops have both left- and right-moving modes, such that they naturally generate a massless field that looks like the Lorentz product of two gauge fields, i.e. like a graviton multiplet.

Let us briefly list the spectrum of closed string theory. It contains the metric, $G^{M N}$, the scalar dilaton $\Phi$, and a two-index antisymmetric tensor $B^{M N}$. Moreover, the GSO projection acts as a chiral projection on the spacetime fermions emerging from each of the left- and rightmoving modes of closed string theory. If the same chirality is projected in each case, then one obtains type-IIA string theory. Its bosonic field content consists of a gauge field $A_{1}$ and a three-form $C_{3}$. If the chiral projections are opposite, then type-IIB theory results, with as bosonic field content a scalar, a two-form $C_{2}$, and a four-form $C_{4}$. Both the type-IIA and the type-IIB theories possess $\mathcal{N}=2$ supersymmetry.

Open strings can also be included into type-II string theory, breaking the supersymmetry to $\mathcal{N}=1$. Interactions can be introduced by allowing the string worldsheet to have holes and handles. The dilaton $\Phi$ 's action measures these topology changes so that the quantity $e^{\Phi}$ plays the role of the theory's coupling. When open and closed string sectors are combined the Yang-Mills coupling from the open string sector has $g_{Y M}^{2}=e^{\Phi}$.

For the AdS/CFT correspondence applied to $(3+1)$ dimensional field theories, ten-dimensional type-IIB string theory is of central importance, and, in particular, its lowenergy limit where strings become point-like and string theory becomes supergravity. There exists no completely satisfactory action for the type-IIB supergravity, since it involves an antisymmetric field $C_{4}$ with self-dual field strength $F_{5}$. However, it is possible to write an action involving both dualities of $C_{4}$, and then impose the selfduality as a supplementary field equation. In this way, one obtains (see, for example, [37-39])

$$
\begin{array}{r}
S_{I I B}=\frac{1}{4 \kappa_{B}^{2}} \int \sqrt{G} e^{-2 \Phi}\left(2 R_{G}+8 \partial_{\mu} \Phi \partial^{\mu} \Phi-\left|H_{3}\right|^{2}\right) \\
-\frac{1}{4 \kappa_{B}^{2}} \int\left[\sqrt{G}\left(\left|F_{1}\right|^{2}+\left|\tilde{F}_{3}\right|^{2}+\frac{1}{2}\left|\tilde{F}_{5}\right|^{2}\right)\right. \\
\left.+C_{4} \wedge H_{3} \wedge F_{3}\right]+ \text { fermions }
\end{array}
$$

where the field strengths are defined by

$$
\begin{aligned}
& F_{1}=\mathrm{d} C, \quad H_{3}=\mathrm{d} B, \quad F_{3}=\mathrm{d} C_{2}, \quad F_{5}=\mathrm{d} C_{4}, \\
& \tilde{F}_{3}=F_{3}-C H_{3}, \quad \tilde{F}_{5}=F_{5}-\frac{1}{2} A_{2} \wedge H_{3}+\frac{1}{2} B \wedge F_{3},
\end{aligned}
$$

and we have the additional self-duality condition $* \tilde{F}_{5}=\tilde{F}_{5}$.

\subsubsection{D branes}

When open strings are included, it turns out to be consistent to introduce the strings in such a way that their end points are restricted to a subspace of the full ten dimensions. The resulting hyperplanes, on which the strings' ends are confined, are called D branes [12-14]. Solitonic solutions of the supergravity actions also exist that are naturally sourced by these branes. In fact, D branes are the fundamental electric and magnetic sources of many of the supergravity antisymmetric forms.

In particular, IIA theory allows branes of even dimension that are electric and magnetic sources for $A_{1}$ and $C_{3}$. IIB theory includes odd-dimension branes that are electric and magnetic sources for the dilaton, two- and four-index fields.

The action for a D brane is given by the Dirac-BornInfeld (DBI) action which is an extension of the NambuGoto form for the fundamental string - one simply minimizes its worldvolume. There are extra terms originating from the role of the D branes as sources for an antisymmetric $(p+1)$-form $F$, including terms of Chern-Simons type. $F$ is the gauge field strength tensor describing gauge fields on the $\mathrm{D}$ brane and $\phi$ the dilaton. The action, in string frame, is

$$
\begin{aligned}
S_{\mathrm{D} p}= & -\mu_{p} \int \mathrm{d}^{(p+1)} \xi e^{-\phi} \\
& \times \sqrt{-\operatorname{det}\left(P\left[G+2 \pi \alpha^{\prime} B\right]_{a b}+2 \pi \alpha^{\prime} F_{a b}\right)} \\
& +\frac{\left(2 \pi \alpha^{\prime}\right)^{2}}{2} \mu_{p} \int P\left[C^{(p+1)}\right] \wedge F \wedge F,
\end{aligned}
$$

where $\mu_{p}=(2 \pi)^{-p} \alpha^{\prime-\frac{(p+1)}{2}}$. Here $B$ is an external antisymmetric two-form which may be present in the supergravity background. In principle, the two-form $B$ may also contribute terms of Chern-Simons form, which are, however, not relevant for the examples described in detail in this review. 


\section{$2.2 \mathcal{N}=4$ super-Yang-Mills theory}

In its original form [15], the AdS/CFT correspondence involves a highly symmetric quantum field theory in $(3+1)$ dimensions, $\mathcal{N}=4 S U(N)$ supersymmetric Yang-Mills theory. The field content of $\mathcal{N}=4$ super-Yang-Mills theory is as follows: There are a gauge field $A_{\mu}$, which is a singlet of the $S U(4)$ global R-symmetry group, four Weyl fermions in the 4 of $S U(4)$, and six real scalars in the $\mathbf{6}$ of $S U(4)$. An important point is that due to the supersymmetry, all these fields are in the adjoint representation of the gauge group $S U(N)$.

This theory naturally arises on the surface of a D3 brane in type-IIB superstring theory. Open strings generate a massless gauge field in ten dimensions. When the open string ends are restricted to a $(3+1)$-dimensional subspace the ten components of the gauge field naturally break into a $(3+1)$-dimensional gauge field and 6 scalar fields. The fermionic superpartners naturally separate to complete the $(3+1)$-dimensional supermultiplets.

The $\mathcal{N}=4$ theory has the property that the beta function of its unique coupling vanishes to all orders in perturbation theory, $\beta=0$. This implies the theory is conformal with conformal symmetry group $S O(4,2)$ also at the quantum level. Moreover, this theory has a global $S U(4)$ R-symmetry group. The complete superconformal group is $S U(2,2 \mid 4)$, of which both $S O(4,2)$ and $S U(4)$ are bosonic subgroups.

\subsection{AdS/CFT correspondence}

The AdS/CFT correspondence was first suggested by Maldacena in 1997 [15], using guiding principles from blackhole physics. The string theory origin of AdS/CFT rests in the fact that D3 branes, i.e. $(3+1)$-dimensional hyperplanes in $(9+1)$-dimensional space, may be interpreted from two different points of view - see fig. 1.

Firstly, D3 branes are hyperplanes in ten-dimensional space on which open strings can end. In the low-energy limit where only massless string degrees of freedom contribute, these open string degrees of freedom correspond to $\mathcal{N}=4$ super Yang-Mills theory with gauge group $U(N)$, where $N$ corresponds to the number of superimposed D3 branes. The gauge group $U(N)$ factorizes into $S U(N) \times U(1)$. The $U(1)$ factor corresponds to the motion of the center of mass of the D3 branes. The global symmetries of the theory are the $S O(4,2)$ superconformal group and the $S U(4)$ R-symmetry (which is isomorphic to $S O(6))$.

On the other hand, D3 branes are also solitonic solutions of ten-dimensional type-IIB supergravity, with a metric of the form

$\mathrm{d} s^{2}=\left(1+\frac{R^{4}}{y^{4}}\right)^{-\frac{1}{2}} \eta_{i j} \mathrm{~d} x^{i} \mathrm{~d} x^{j}+\left(1+\frac{R^{4}}{y^{4}}\right)^{\frac{1}{2}}\left(\mathrm{~d} y^{2}+y^{2} \mathrm{~d} \Omega_{5}{ }^{2}\right)$.

Here

$$
R^{4}=4 \pi g_{s} N \alpha^{\prime 2}
$$



Fig. 1. Schematic representation of the AdS/CFT duality. The D3 branes warp the space into a throat whose near-horizon geometry is $A d S_{5} \times S^{5}$. Asymptotically, far away from the branes the geometry returns to flat ten-dimensional space. Open string degrees of freedom on the D3 branes, which give rise to $\mathcal{N}=4$ $S U(N)$ super Yang-Mills theory, are mapped to closed string excitations in the $A d S_{5} \times S^{5}$ near-horizon geometry.

where $\lambda=g_{s} N=g_{Y M}^{2} N$ is the 't Hooft coupling, $N$ the number of D3 branes and $\alpha^{\prime}$ the inverse string tension $\left(\alpha^{\prime}=l_{s}^{2}, l_{s}\right.$ string length). $\eta_{i j}$ is the standard $(3+1)$ dimensional Minkowski metric and the $x^{i}$ are the coordinates on the stack of D3 branes. $\vec{y}$ denotes the six spatial coordinates perpendicular to the brane, $y \equiv \sqrt{y_{M} y^{M}}$. For $y \gg R$ this metric returns to flat $(9+1)$-dimensional Minkowski space. On the other hand, in the near-horizon limit $y \ll R$, which is again a low-energy limit, we perform a coordinate transformation

$$
u \equiv \frac{R^{2}}{y}
$$

and obtain from $(2.7)$

$$
\mathrm{d} s^{2}=R^{2}\left(\frac{1}{u^{2}} \eta_{i j} \mathrm{~d} x^{i} \mathrm{~d} x^{j}+\frac{\mathrm{d} u^{2}}{u^{2}}+\mathrm{d} \Omega_{5}{ }^{2}\right),
$$

which is the metric of $A d S_{5} \times S^{5}$, with $A d S_{5}$ the fivedimensional anti-de-Sitter space $\mathrm{d} s^{2}=\frac{R^{2}}{u^{2}}\left(\eta_{i j} \mathrm{~d} x^{i} \mathrm{~d} x^{j}+\right.$ $\left.\mathrm{d} u^{2}\right)$. Here $R$ is the anti-de-Sitter radius. Anti-de-Sitter space has negative constant curvature $\mathcal{R}=-\frac{d(d-1)}{R^{2}}$, and a boundary at $u=0$.

A further ingredient is that D3 branes carry charge that sources a four-form antisymmetric tensor field $C_{4}$ in IIB supergravity. The D3 brane supergravity solution also therefore has a self-dual five-form field $F_{5}=\mathrm{d} C_{4}$, which satisfies

$$
\int_{S^{5}} F_{5}=N
$$

The isometry of the space $A d S_{5}$ is $S O(4,2)$ (it can be constructed as a surface embedded in a $(4+2)$-dimensional space). The isometry of the five sphere is $S O(6)$. This product group matches the maximal bosonic subgroup of the supergroup $S U(2,2 \mid 4)$, which encodes the symmetries of the $\mathcal{N}=4$ supersymmetric field theory. Note, in particular, that $S O(6) \simeq S U(4)$, which is the R-symmetry group of $\mathcal{N}=4$ supersymmetry. Since the global symmetries match it is possible to consider that these two 
theories are dual. Note that the gauge symmetry of the gauge theory is considered a redundant symmetry that is not manifest in any gauge invariant observable.

The conjecture of Maldacena consists in identifying the two low-energy theories, i.e. $\mathcal{N}=4 S U(N)$ YangMills theory, and string theory on $A d S_{5} \times S^{5}$. There are three different versions of this conjecture, depending on the precise form of the limits taken. The strongest form of the conjecture states that the correspondence between $\mathcal{N}=4 S U(N)$ Yang-Mills theory, and string theory on $A d S_{5} \times S^{5}$, is valid in general. It is not possible to test this strongest version of the correspondence today since it is not known how to quantize string theory on curved space backgrounds with Ramond-Ramond flux. The second form of the correspondence restricts the duality to the 't Hooft limit, in which $N \rightarrow \infty$, while $\lambda=g_{Y M}^{2} N$ is kept fixed. In this case only planar diagrams contribute on the field theory side, while the string theory on $A d S_{5} \times S^{5}$ is restricted to the semi-classical limit in which the string coupling $g_{s} \equiv g_{Y M}^{2}$ goes to zero. Finally, the third form of the correspondence specializes further to the case in which $\lambda$ is large. In this limit, strongly coupled $\mathcal{N}=4$ $S U(N)$ Yang-Mills theory is mapped to supergravity on $A d S_{5} \times S^{5}$; the inverse string tension $\alpha^{\prime}$ goes to zero. In this paper we will be dealing with this third form of the correspondence. Equation (2.8) implies that the AdS radius $R$ remains finite when $\lambda$ is large and fixed, $N \rightarrow \infty$, and $\alpha^{\prime}$ is small.

The gravitational side of the correspondence has an extra non-compact direction, $y$, relative to the gauge theory and so the correspondence is described as being holographic [40] - the contents of the $(4+1)$-dimensional theory are encoded by the degrees of freedom in the $(3+1)$-dimensional gauge theory. To understand what this extra direction is in the gauge theory it is useful to look at the action of dilatations (a subgroup of $S O(2,4)$ ). The action of a massless scalar in $(3+1)$ dimensions is invariant under

$$
S=\int \mathrm{d}^{4} x(\partial \phi)^{2}, \quad x \rightarrow e^{\alpha} x, \phi \rightarrow e^{-\alpha} \phi,
$$

with $\alpha$ some arbitrary parameter. The power of the scaling here tells us that $\phi$ has energy dimension one and $x$ inverse energy dimensions. On the gravitational side of the dual this symmetry is a symmetry of the metric (note supergravity fields do not transform) and for (2.10) to be invariant we require

$$
y \rightarrow e^{-\alpha} y
$$

We have learnt that the radial direction in AdS scales like a scalar field under the gauge theory's dilatations and hence is an energy scale. This leads to the natural interpretation that the holographic direction is a representation of the renormalization group scale in the gauge theory.

The AdS/CFT correspondence has been developed further in $[31,41]$ where a field operator map has been established. This maps gauge invariant operators of the $\mathcal{N}=4$ Yang-Mills theory in a particular irreducible representation of $S U(4)$ to supergravity fields in the same representation. These five-dimensional supergravity fields are obtained by Kaluza-Klein reduction of the original ten-dimensional supergravity fields on the five-sphere $S^{5}$. Consider a scalar field in AdS with action

$$
S=\int \mathrm{d}^{4} x \mathrm{~d} u \sqrt{-g}\left(g^{a b} \partial_{a} \phi \partial_{b} \phi-m^{2} \phi^{2}\right),
$$

where $g$ is the determinant of the metric. The solutions of the equation of motion are of the form

$$
\phi(u) \sim u^{4-\Delta} \phi_{0}+u^{\Delta}\langle\mathcal{O}\rangle
$$

with $m^{2}=\Delta(\Delta-4)$. Since the supergravity field does not transform under the field theory dilatations and $u$ is an inverse mass scale, we see that $\phi_{0}$ and $\langle\mathcal{O}\rangle$ carry dimension $(4-\Delta)$ and $\Delta$, respectively. Therefore, as discussed in [41], the boundary value $\phi_{0}$ may be identified with the source of the gauge theory operator $\mathcal{O}$, and $\langle\mathcal{O}\rangle$ is the vev (vacuum expectation value) of $\mathcal{O}$.

The AdS/CFT correspondence may then be stated as follows:

$$
\left\langle e^{\int \mathrm{d}^{d} x \phi_{0}(\vec{x}) \mathcal{O}(\vec{x})}\right\rangle_{C F T}=\left.Z_{\text {sugra }}\right|_{\phi(0, \vec{x})=\phi_{0}(\vec{x})},
$$

i.e. the generating functional of particular gauge-invariant operators in the conformal field theory coincides with the generating functional for tree diagrams in supergravity, with the boundary values of the supergravity fields coinciding with the sources.

This suggests that the AdS/CFT correspondence may be tested by comparing correlation functions of $\mathcal{N}=4$ quantum field theory with classical correlation functions on $A d S_{5}$. This is not possible in general for any correlation function even in the large- $N$ limit, since in the Maldacena limit, the supergravity dual describes $S U(N) \mathcal{N}=4$ super-Yang-Mills at strong coupling. However, for selected correlation functions which satisfy non-renormalization theorems such that they are independent of the coupling, direct comparison is possible. This applies, in particular, to the two- and three-point functions of $1 / 2$ BPS operators $[42,43]$. These operators are annihilated by half of the supersymmetry generators. Another beautiful test of the AdS/CFT correspondence is the calculation of the conformal trace anomaly of $\mathcal{N}=4$ theory from $A d S_{5} \times S^{5}$ supergravity [44].

Let us conclude this introduction to AdS/CFT by noting that up to the present day, there is no proof of the AdS/CFT correspondence taking account of its stringtheoretical origin. However, in the weakest of its three forms as discussed above, the huge amount of symmetry present almost guarantees that the AdS/CFT correspondence should hold. When proceeding to less symmetrical situations below, generalized gauge/gravity dualities remain a conjecture though.

\subsection{Holographic RG flows}

A necessary ingredient for obtaining gravity duals of more QCD-like theories than $\mathcal{N}=4$ super-Yang-Mills theory is to generalize the correspondence to non-conformal 
field theories with less or no supersymmetry, which have a renormalization group flow. In particular, to obtain theories with a running coupling it is necessary to deform the five-dimensional AdS space, which has isometry $S O(4,2)$ [45]. This symmetry corresponds to conformal symmetry in the dual field theory and thus to a renormalization group fixed point. The simplest way to do this is to switch on supergravity fields in the bulk which backreact on the metric. The analysis of a scalar field in (2.15) is in fact only an asymptotic solution ignoring the backreaction on the metric. If we switch on a supergravity field the UV (small $u$ ) behaviour will be that in (2.15) - so we can identify the deforming operator present. Generically, in the interior of the space, the geometry will deform from AdS indicating the loss of conformality.

The simplest example of such a deformation is the multi-centre D3 brane solution $[15,46,47]$. These are geometries with a stack of parallel D3 branes present but where the D3 are separated in the six-dimensional space transverse to their worldvolume

$$
\begin{aligned}
\mathrm{d} s^{2} & =H^{-1 / 2} \mathrm{~d} x^{2}+H^{1 / 2}\left(\mathrm{~d} y^{2}+\mathrm{d} \Omega_{5}^{2}\right), \\
C_{4} & =H^{-1} \mathrm{~d} x^{0} \wedge \ldots \mathrm{d} x^{3}
\end{aligned}
$$

with

$$
H=1+\sum_{\mathrm{D} 3}\left(\frac{1}{\left|y-y_{\mathrm{D} 3}\right|^{4}}\right)
$$

The $y_{\mathrm{D} 3}$ are the positions of the D3 branes.

The function $H$ can be expanded in terms of spherical harmonics [46] on the five sphere, labelled by their representation of $S O(6)$, as follows:

$$
H \simeq \frac{R^{4}}{y^{4}}\left(\ldots+\alpha y^{4}+1+\frac{\beta}{y^{2}} Y_{20}+\frac{\gamma}{y^{4}} Y_{50}+\ldots\right)
$$

Each of $\alpha, \beta, \ldots$ is a deformation of the geometry from $\mathrm{AdS}$ and has a corresponding interpretation as a deformation of the gauge theory. They correspond to operator vevs in the dual field theory, which have been determined using the symmetries of the set-up in $[48,49]$. Here we consider the following example: $\beta$ must carry field theory energy dimension of two (to cancel that of $y$ ) and be in the 20-dimensional representation of $S O(6)$. There is indeed such an operator in the field theory, $\operatorname{Tr} \phi^{2}$. Similarly, $\gamma$ matches to $\operatorname{Tr} \phi^{4}$. Note these operator vevs are relevant operators and are unimportant at large $y$ (the UV), but grow in importance into the IR (small $y$ ).

The field theory intepretation of the parameter $\alpha$, which corresponds to leaving the near-horizon limit of the geometry, has already been found in [50-52]. Again from the symmetries we see that it must be an R-charged singlet and of dimension - 4 -it corresponds to the coupling of the interaction term $G \operatorname{Tr} F^{4}$. This is an irrelevant operator whose influence is in the UV (at large $y$ ), where it grows.

These multi-centre geometries have been explicitly constructed as a supergravity renormalization group flow in [53].

Other more complicated examples of holographic RG flows exist in the literature [54-57]. Generically, the more supersymmetry is retained the more checks of their agreement with field theory exist. For example, a flow to an $\mathcal{N}=2$ theory can be found by giving equal mass to four of the six scalars and two of the four gauginos of the $\mathcal{N}=4$ theory [58-60]. This theory is called the $\mathcal{N}=2^{*}$ theory and the induced flow of the dilaton can be matched to the expected running coupling behaviour of the field theory $[61,62]$. A general field-theoretical interpretation of holographic RG flows is given in $[63,64]$.

Flows to $\mathcal{N}=1[65-67]$ and $\mathcal{N}=0$ theories [52,68-71] also exist in the literature. We will introduce those which are used below in the appropriate sections of the text.

\subsection{Confinement}

The confinement of quarks and gluons within hadrons is a crucial aspect of QCD and more generically strongly coupled gauge dynamics. There has been considerable work on how the AdS/CFT correspondence incorporates confinement which we will briefly review here.

\subsubsection{Heavy-source interaction energy}

The simplest analysis is to look at the interaction energy between two very heavy static quarks at different separations (field theoretically this is related to the area or perimeter law of a Wilson loop). In $[72,73]$ heavy quarks were introduced into the AdS/CFT correspondence by placing a probe (i.e. non-backreacting) D3 brane at large radius in AdS (large $y$ in the discussion above - the field theory UV). Strings stretched from the probe D3 to the $N$ D3 at the origin are formally very massive gauge bosons associated with the breaking of the gauge symmetry in the pattern $S U(N+1) \rightarrow S U(N)$ by a vev for one of the six adjoint scalars. One can think of these strings equally as heavy sources though since they are massive objects in the fundamental representation of $S U(N)$.

When one includes two such strings to represent a quark and an antiquark there can be two possible configurations - see fig. 2. Each string could lie straight in the space between the probe and the central D3 stack. There would then be no interaction between the quarks (neglecting the exchange of supergravity fields). Alternatively, it might be energetically favourable for the strings to join, in which case their energy would depend on the separation of the quarks on the boundary probe.

In pure AdS a simple computation with the NambuGoto action of the string [72] determines the preferred configuration. The strings indeed connect and one finds that the energy of the configuration is given by

$$
E=-\frac{4 \pi^{2}\left(2 g_{Y M}^{2} N\right)^{1 / 2}}{\Gamma(1 / 4)^{4} L}
$$

with $L$ the quark separation. Note that the inverse proportionality to $L$ was guaranteed here by the conformal symmetry of the gauge theory. One finds that as the quarks are 


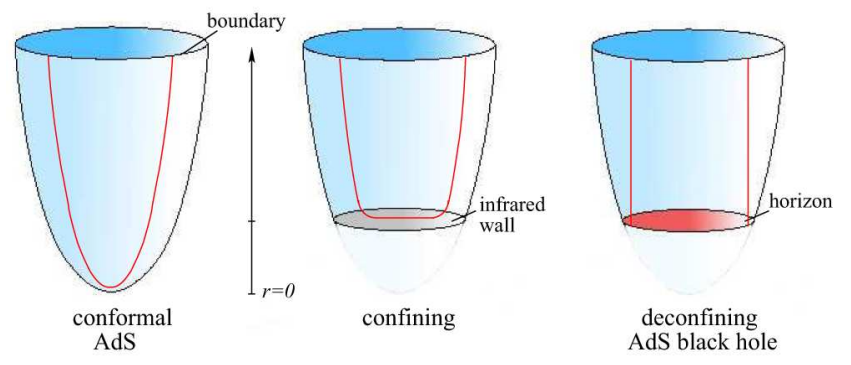

Fig. 2. Three important configurations of strings connecting sources on a probe brane. In AdS the strings from two sources prefer to join than fall independently to $r=0$. They dip further into the space the more the quarks are separated. In a confining geometry a blockage forces the string to lie along the blockage and the energy scales with the separation. Finite temperature is reproduced by a black-hole horizon - once the strings fall in, the quarks are screened from each other.

separated further, the string connecting them dips further into the AdS space.

Non-conformal gauge theories such as those induced by a deformation will be described by some deformed AdS space and the relation between energy and length can change radically. The simplest toy understanding of how confinement sets in is as follows. A mass gap in the theory will be represented by a block in the space stopping the supergravity fields accessing values of the radius (the renormalization group scale) below that mass gap energy. Example blockages are divergences of the supergravity fields or the presence of branes completing the solution below the gap radius. In these cases the string connecting two heavy sources will behave for small separations as in AdS but as the quarks are separated and the strings dip deeper into the interior of the space they will eventually hit the blockage. At this point they have little alternative than to lie along the blockage. Now separating the sources further simply extends the string along the blockage and the energy must be proportional to the separation $L$. This behaviour is confinement.

Another useful example is to consider the effects of finite temperature in this system. If one heats up a gauge theory above the scale of its mass gap one expects the theory to deconfine. In the dual gravity description, finite temperature is associated with the presence of a black hole in the AdS space [31,32]. A black hole has all the associated thermodynamic properties (e.g., temperature and entropy) to be dual to the equivalent properties of the thermal bath in the field theory. The position of the black-hole's horizon in the radial direction again cuts off the space at low energies corresponding to energies below the temperature scale. Consider again the linked string between two heavy sources as we separate them - now as the string dips deeper into the space, it will encounter the horizon. The string must fall into the black hole and we are left with two disconnected strings from each source to the horizon. We see that the quarks are screened from each other since they can now be moved independently.

\subsubsection{A discrete glueball spectrum}

Another clear signal that a theory is confining is if there is a discrete spectrum of bound states. Below we will discuss in detail mesons in theories with quarks. Let us briefly review here though how a discrete glueball spectrum emerges in a gravity dual.

We will look for glueballs associated with the gauge field operator $\operatorname{Tr} F^{2}[31,74]$. In the AdS/CFT correspondence $\operatorname{Tr} F^{2}$ (conformal dimension $\Delta=4$ ) is associated with a massless scalar (the dilaton) with an equation of motion

$$
\partial_{r} \sqrt{-g} g^{r r} \partial_{r} \phi+\sqrt{-g} g^{x x} \partial_{x}^{2} \phi=0 .
$$

We will look for glueballs as solutions of the form

$$
\phi(r, x)=\phi(r) e^{i k \cdot x}, \quad-k^{2}=M^{2} .
$$

In other words we are looking for pure momentum planewave excitations of $\operatorname{Tr} F^{2}$. To find a discrete spectrum we would want the solutions for $\phi(r)$ only to exist (to be normalizable on the space) for discrete values of the glueball mass $M$.

Generically, in a deformed geometry the metric can be written in the form

$$
\mathrm{d} s_{5}{ }^{2}=e^{2 A(u)} \eta_{i j} \mathrm{~d} x^{i} \mathrm{~d} x^{j}+\mathrm{d} u^{2} .
$$

To recover AdS one sets $\exp (A)=R / u . u \rightarrow 0$ is the UV and $u \rightarrow \infty$ the IR. In a deformed geometry $A$ will deviate from the AdS value as one moves into the IR.

Now, if we make the transformation $\phi=e^{-3 A / 2} \psi$ on the dilaton's equation of motion it takes the form

$$
\left(-\partial_{r}^{2}+V(r)\right) \psi=M^{2} \psi, \quad V=\frac{3}{2} A^{\prime \prime}+\frac{9}{4}\left(A^{\prime}\right)^{2},
$$

which is a Schrödinger equation with energy $M^{2}$.

In pure AdS the potential is given by $V \sim 1 / u^{2}$ and the spectrum of the Schrödinger equation is continuous - we expect this in a conformal gauge theory. For a confining geometry though we expect $A(u)$ to diverge at large $u$ creating a "hard wall", at some $u=u_{0}$, as discussed in the previous subsection. The potential in the Schrödinger equation is now a well and we expect a discrete energy spectrum. The glueball spectrum of a theory with a mass gap of this type in the gravity dual is very likely to generate a discrete glueball spectrum therefore.

\section{AdS/CFT with flavour}

The original AdS/CFT correspondence only involves fields in the adjoint representation of the gauge group. To generalize the correspondence to quark degrees of freedom, which are in the fundamental representation of the gauge group, additional ingredients are necessary. The simplest thing is to add a new type of brane into the configuration in addition to the D3 branes. The open strings with both ends on the D3 generate the adjoint degrees of freedom. Strings between the D3 and the new brane have only 


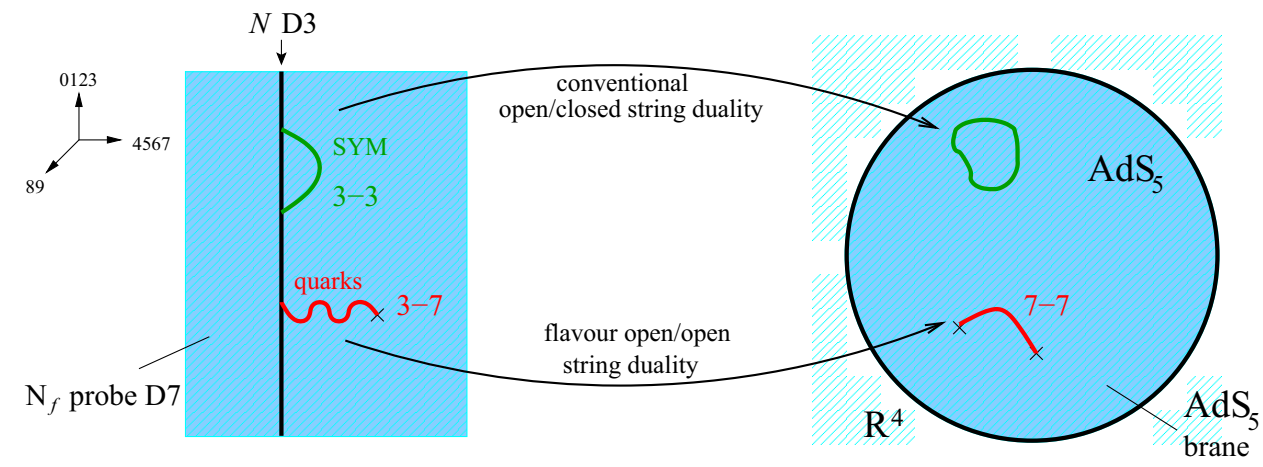

Fig. 3. Schematic representation of the AdS/CFT duality with added flavour. In addition to the original AdS/CFT duality, open string degrees of freedom representing quarks are mapped to open strings beginning and ending on the D7 probe, which asymptotically near the boundary wrap $A d S_{5} \times S^{3}$ inside $A d S_{5} \times S^{5}$. For simplicity, the five sphere is not drawn in this picture.

one end on the $N$ D3 branes and hence generate matter in the fundamental representation. Such matter will typically come in quark supermultiplets because of the underlying supersymmetry of the string theory. If supersymmetry is broken one expects the scalar squarks to become massive on the scale of the supersymmetry breaking whilst the fermionic quarks will be kept massless by their chiral symmetries.

If the new branes can be separated from the D3 branes in some direction transverse to both branes, then the minimum length string between the two branes has non-zero energy (length times tension) and hence the quark is massive $\left(m_{q}=L / 2 \pi \alpha^{\prime}\right)$.

Strings with both ends on the flavour brane are in the adjoint of the $U\left(N_{f}\right)$ flavour symmetry of the quarks and hence naturally describe mesonic degrees of freedom. In string theory these states describe fluctuations of the brane in the background geometry. Small oscillations of the branes are therefore dual to the gauge theory mesons.

The need for separating the probe from the D3 brane stack excludes D9 branes as probes. D3 and D5 brane probes lead to defect field theories discussed below in sect. 5.4, if supersymmetry is to be preserved. This leaves D7 brane probes for adding flavour to a $(3+1)$-dimensional field theory.

\subsection{The D3/D7 brane intersection}

The simplest way to obtain quark bilinear operators within gauge/gravity duality is to add D7 branes [16, 27, $28]$. The D7 branes are added in such a way that they extend in space-time as given in table 1 , where 0 is the time direction. We thus consider a stack of $N$ coincident D3 branes (along 0123) which is embedded into the worldvolume of $N_{f}$ D7 (probe) branes (along 01234567), as shown (on the 1.h.s. of) fig. 3 .

The D3/D7 brane intersection preserves $1 / 4$ of the total amount of supersymmetry in type-IIB string theory (corresponding to 8 supercharges) and has an $S O(4) \times$ $S O(2)$ isometry in the directions transverse to the D3 branes. The $S O(4)$ rotates in $x^{4}, x^{5}, x^{6}, x^{7}$, while the $S O(2)$ group acts on $x^{8}, x^{9}$. Note that separating the D3
Table 1. The D3/D7 brane intersection in $(9+1)$-dimensional flat space.

\begin{tabular}{|c|c|c|c|c|c|c|c|c|c|c|}
\hline & 0 & 1 & 2 & 3 & 4 & 5 & 6 & 7 & 8 & 9 \\
\hline D3 & $\mathrm{X}$ & $\mathrm{X}$ & $\mathrm{X}$ & $\mathrm{X}$ & & & & & & \\
\hline $\mathrm{D} 7$ & $\mathrm{X}$ & $\mathrm{X}$ & $\mathrm{X}$ & $\mathrm{X}$ & $\mathrm{X}$ & $\mathrm{X}$ & $\mathrm{X}$ & $\mathrm{X}$ & & \\
\hline
\end{tabular}

branes from the D7 branes in the 89-direction by a distance $L$ explicitly breaks the $S O(2)$ group. These geometrical symmetries are also realized in the dual field theory.

\subsubsection{Field theory of the D3/D7 brane intersection}

The field theory corresponding to this brane set-up is a $\mathcal{N}=2$ supersymmetric $U(N)$ gauge theory which, in addition to the degrees of freedom of $\mathcal{N}=4$ super-Yang-Mills, contains $N_{f}$ hypermultiplets in the fundamental representation of the gauge group.

This particular field theory arises as follows. The $\mathcal{N}=4$ super Yang-Mills multiplet is generated by massless open string modes on the D3 branes (3-3 strings), whereas the $\mathcal{N}=2$ hypermultiplets descend from strings stretching between the D3 and the D7 branes (3-7 strings), cf. fig. 3 . We take a limit in which the 7-7 strings decouple, leaving a purely four-dimensional theory. This decoupling is achieved by taking the usual large- $N$ limit while keeping the four-dimensional 't Hooft coupling $\lambda=g_{Y M}^{2} N=g_{s} N$ and $N_{f}$ fixed. The eight-dimensional 't Hooft coupling $\lambda^{\prime}$ for the $N_{f}$ D7 branes is $\lambda^{\prime}=\lambda\left(2 \pi l_{s}\right)^{4} N_{f} / N$ which vanishes in the low-energy $\alpha^{\prime} \rightarrow 0$ (i.e., $l_{s} \rightarrow 0$ ) limit. The 7-7 strings therefore do not interact with the other $(3-3,3-7)$ strings anymore, and the $U\left(N_{f}\right)$ gauge group on the D7 branes plays the role of a global flavour group in the fourdimensional theory.

The Lagrangian of the $\mathcal{N}=2$ worldvolume theory can conveniently be written down in $\mathcal{N}=1$ superspace formalism. Under $\mathcal{N}=1$ supersymmetry the $\mathcal{N}=4$ vector multiplet decomposes into the vector multiplet $W_{\alpha}$ and the three chiral superfields $\Phi_{1}, \Phi_{2}, \Phi_{3}$. The $\mathcal{N}=2$ fundamental hypermultiplets can be written in terms of the $\mathcal{N}=1$ chiral multiplets $Q^{r}, \tilde{Q}_{r}\left(r=1, \ldots, N_{f}\right)$. The La- 
Table 2. Fields of the D3/D7 low-energy effective field theory and their quantum numbers under the global symmetries. Note that $U(1)_{B} \subset U\left(N_{f}\right)$.

\begin{tabular}{|c|c|c|c|c|c|c|c|}
\hline $\mathcal{N}=2$ & Components & Spin & $S U(2)_{\Phi} \times S U(2)_{\mathcal{R}}$ & $U(1)_{\mathcal{R}}$ & $\Delta$ & $U\left(N_{f}\right)$ & $U(1)_{B}$ \\
\hline$\left(\Phi_{1}, \Phi_{2}\right)$ & $X^{4}, X^{5}, X^{6}, X^{7}$ & 0 & $\left(\frac{1}{2}, \frac{1}{2}\right)$ & 0 & 1 & 1 & 0 \\
Hyper & $\lambda_{1}, \lambda_{2}$ & $\frac{1}{2}$ & $\left(\frac{1}{2}, 0\right)$ & -1 & $\frac{3}{2}$ & 1 & 0 \\
\hline$\left(\Phi_{3}, W_{\alpha}\right)$ & $X_{V}^{A}=\left(X^{8}, X^{9}\right)$ & 0 & $(0,0)$ & +2 & 1 & 1 & 0 \\
Vector & $\lambda_{3}, \lambda_{4}$ & $\frac{1}{2}$ & $\left(0, \frac{1}{2}\right)$ & +1 & $\frac{3}{2}$ & 1 & 0 \\
& $v_{\mu}$ & 1 & $(0,0)$ & 0 & 1 & 1 & 0 \\
\hline$(Q, \tilde{Q})$ & $q^{m}=(q, \overline{\tilde{q}})$ & 0 & $\left(0, \frac{1}{2}\right)$ & 0 & 1 & $N_{f}$ & +1 \\
Fund. hyper & $\psi_{i}=\left(\psi, \tilde{\psi}^{\dagger}\right)$ & $\frac{1}{2}$ & $(0,0)$ & $\mp 1$ & $\frac{3}{2}$ & $N_{f}$ & +1 \\
\hline
\end{tabular}

grangian is thus given by

$$
\begin{aligned}
\mathcal{L}=\operatorname{Im}[ & \tau \int \mathrm{d}^{4} \theta\left(\operatorname{tr}\left(\bar{\Phi}_{I} e^{V} \Phi_{I} e^{-V}\right)+Q_{r}^{\dagger} e^{V} Q^{r}+\tilde{Q}_{r}^{\dagger} e^{-V} \tilde{Q}^{r}\right) \\
& \left.+\tau \int \mathrm{d}^{2} \theta\left(\operatorname{tr}\left(W^{\alpha} W_{\alpha}\right)+W\right)+c . c .\right]
\end{aligned}
$$

where the superpotential $W$ is

$$
W=\operatorname{tr}\left(\varepsilon_{I J K} \Phi_{I} \Phi_{J} \Phi_{K}\right)+\tilde{Q}_{r}\left(m_{q}+\Phi_{3}\right) Q^{r},
$$

and $\tau$ is the complex gauge coupling. The beta function of this theory is $\beta \propto \lambda^{2} N_{f} / N$, which goes to zero for $N_{f}$ small, fixed 't Hooft coupling $\lambda$ and $N \rightarrow \infty$, such that the theory remains conformal in this limit.

The components of the $\mathcal{N}=1$ superfields and their quantum numbers are summarized in table 2 (see also [75]). We will need them for the construction of operators. The $S O(2) \simeq U(1)$ isometry corresponds to a $U(1)_{R}$ R-symmetry in the field theory - note that it is explicitly broken by a quark mass $m_{q} \propto L$. The field theory has also a global $S O(4) \approx S U(2)_{\Phi} \times S U(2)_{\mathcal{R}}$ symmetry which consists of a $S U(2)_{\Phi}$ symmetry and a $\mathcal{N}=2$ $S U(2)_{\mathcal{R}}$ R-symmetry. The global symmetry $S U(2)_{\Phi}$ rotates the scalars in the adjoint hypermultiplet. There is also a baryonic $U(1)_{B}$ which is a subgroup of the $U\left(N_{f}\right)$ flavour group. The fundamental superfields $Q^{r}\left(\tilde{Q}_{r}\right)$ are charged $+1(-1)$ under $U(1)_{B}$, while the adjoint fields are inert.

\subsection{The probe brane correspondence}

The simplest way to analyze the D3/D7 system is to work in the limit where the D7 branes are treated as probes [16]. The term "brane probe" [14] refers to the fact that only a very small number of D7 branes is added, while the number of D3 branes, $N$, which also determines the rank of the gauge group $S U(N)$, goes to infinity. In this limit we neglect the backreaction of the D7 branes on the geometry. Naively, it seems peculiar to treat the large brane as the probe but here one is working in the $N \rightarrow \infty$ limit so the D3 branes can dominate. The limit is clearest on the field theory side: the geometry represents the gauge configuration in which the quarks move. If we neglect the
D7 effects we are simply dropping quark loops from the gauge background which is simply quenching the gauge theory. In sect. 4.1 we will discuss the D3/D7 brane configuration for finite $\frac{N_{f}}{N}$ including the backreaction of the flavour branes.

On the supergravity side of the duality, the $\mathcal{N}=4$ degrees of freedom are described by supergravity on $A d S_{5} \times S^{5}$ as before. However, in addition, there are new degrees of freedom corresponding to the D7 brane probe within the ten-dimensional curved space. The low-energy degrees of freedom of this brane are described by the Dirac-BornInfeld action as described below. These correspond to open string fluctuations on the D7 probe. It turns out, as we will show shortly, that the minimum action configuration for the D7 brane probe corresponds to a probe configuration which asymptotically near the boundary wraps an $A d S_{5} \times S^{3}$ subspace of $A d S_{5} \times S^{5}$.

As shown in fig. 3, the new duality conjectured in [16] is an open-open string duality, as opposed to the original AdS/CFT correspondence which is an open-closed string duality. The duality states that, in addition to the original AdS/CFT duality, gauge invariant field theory operators involving fundamental fields are mapped to fluctuations of the D7 brane probe inside $A d S_{5} \times S^{5}$. This is also shown in fig. 3 .

Let us determine the D7 embedding explicitly. The dynamics of the D7 brane probe is described by the combined Dirac-Born-Infeld and Chern-Simons actions,

$$
\begin{aligned}
S_{\mathrm{D} 7}= & -\mu_{7} \int \mathrm{d}^{8} \xi \sqrt{-\operatorname{det}\left(P[G]_{a b}+2 \pi \alpha^{\prime} F_{a b}\right)} \\
& +\frac{\left(2 \pi \alpha^{\prime}\right)^{2}}{2} \mu_{7} \int P\left[C^{(4)}\right] \wedge F \wedge F .
\end{aligned}
$$

$\mu_{7}=\left[(2 \pi)^{7} g_{s} \alpha^{\prime 4}\right]^{-1}$ is the D7 brane tension and $P$ denotes the pullback of a bulk field to the worldvolume of the brane. $F_{a b}$ is the world-volume field strength. The D7 brane action also contains a fermionic term $S_{\mathrm{D} 7}^{f}$ which will be discussed in sect. 3.3.2.

If we write the $A d S_{5} \times S^{5}$ metric in the form

$$
\mathrm{d} s^{2}=\frac{r^{2}}{R^{2}} \eta_{i j} \mathrm{~d} x^{i} \mathrm{~d} x^{j}+\frac{R^{2}}{r^{2}}\left(\mathrm{~d} \rho^{2}+\rho^{2} \mathrm{~d} \Omega_{3}^{2}+\mathrm{d} w_{5}^{2}+\mathrm{d} w_{6}^{2}\right),
$$


with $\rho^{2}=w_{1}^{2}+\ldots+w_{4}^{2}, r^{2}=\rho^{2}+w_{5}^{2}+w_{6}^{2}$, then the action for a static D7 embedding (with $F_{a b}$ zero on its worldvolume) is given up to angular factors from (3.3) by

$$
S_{\mathrm{D} 7}=-\mu_{7} \int \mathrm{d}^{8} \xi \rho^{3} \sqrt{1+\dot{w}_{5}^{2}+\dot{w}_{6}^{2}}
$$

where a dot indicates a $\rho$ derivative $\left(e . g \cdot \dot{w}_{5} \equiv \partial_{\rho} w_{5}\right)$. The ground-state configuration of the D7 brane then corresponds to the solution of the equation of motion

$$
\frac{\mathrm{d}}{\mathrm{d} \rho}\left[\frac{\rho^{3}}{\sqrt{1+\dot{w}_{5}^{2}+\dot{w}_{6}^{2}}} \frac{\mathrm{d} w}{\mathrm{~d} \rho}\right]=0,
$$

where $w$ denotes either $w_{5}$ or $w_{6}$. Clearly, the action is minimized by $w_{5}, w_{6}$ being any arbitrary constant. The D7 brane probe therefore lies flat in the space. The choice of the position in the $\left(w_{5}, w_{6}\right)$-plane corresponds to choosing the quark mass in the gauge theory action. That $w_{5}, w_{6}$ is constant at all values of $\rho$ is a statement of the nonrenormalization of the mass. The coordinate $\rho$ is a holographic radial direction of the background AdS space and therefore corresponds to the renormalization group scale. The non-renormalization of the mass is an expected characteristic of supersymmetric gauge theories.

In general, the equations of motion have asymptotic $(\rho \rightarrow \infty)$ solutions of the form

$$
w=L+\frac{c}{\rho^{2}}+\ldots
$$

$L$ corresponds to the quark mass as discussed. In agreement with the AdS/CFT result (2.15), the extra parameter $c$ must correspond to the vev of an operator with the same symmetries as the mass and of dimension three (since $\rho$ carries energy dimension). $c$ is therefore a measure of the quark condensate $\left(\bar{q}_{L} q_{R}\right.$; more formally it corresponds to $\partial \mathcal{L} / \partial m$, which includes scalar squark terms, but we assume that the squarks have zero vev here). Moreover, note that solutions with non-zero $c$ are not regular in AdS space and these solutions are excluded. This corresponds to a vev for this operator being forbidden by supersymmetry -it is an F-term of a chiral superfield ${ }^{3}$. A detailed discussion of relation between the asymptotic behaviour (2.15) and (3.7) was given in [76] in the context of "holographic renormalization" [77].

A particularly interesting feature arises if the D7 brane probe is separated from the stack of D3 branes in either the $w^{5}$ or $w^{6}$ directions, where the indices refer to the coordinates given in (3.4). This corresponds to giving a mass to the fundamental hypermultiplet. In this case the radius of the $S^{3}$ becomes a function of the radial coordinate $r$ in $A d S_{5}$. At a radial distance from the deep interior of the AdS space given by the hypermultiplet mass, the radius of the $S^{3}$ shrinks to zero. From a five-dimensional AdS point

\footnotetext{
${ }^{3}$ For $m_{q} \neq 0$, consider the term $m_{q} \psi \tilde{\psi}$ which is the F-term of $m_{q} Q \tilde{Q}$. Supersymmetry is broken, if $c=\langle\psi \tilde{\psi}\rangle \neq 0$. Vice versa, if supersymmetry is preserved, then $c=0$ and the embedding profile must be flat.
}

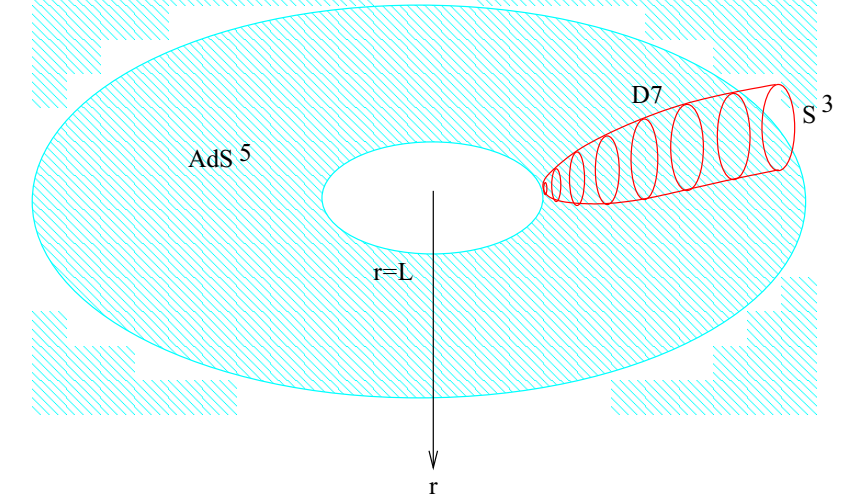

Fig. 4. Gravity dual in the presence of a fundamental hypermultiplet with finite mass $m$. The D7 brane probe is shown in blue. In this case, the radius of the $S^{3}$ wrapped by the D7 brane probe becomes a function of the AdS radial direction $r$. At $r=L$, the radius of the $S^{3}$ shrinks to zero, and the D7 probe does not extend any further into the interior of AdS space. (Figure by Zachary Guralnik, from [18].)

of view, the D7 brane probe seems to "end" at this value of the AdS radial coordinate, as shown in fig. 4 .

This can be seen as follows. According to [17], the induced metric on the D7 brane worldvolume is

$\mathrm{d} s^{2}=\frac{\rho^{2}+L^{2}}{R^{2}} \eta_{i j} \mathrm{~d} x^{i} \mathrm{~d} x^{j}+\frac{R^{2}}{\rho^{2}+L^{2}} \mathrm{~d} \rho^{2}+\frac{R^{2} \rho^{2}}{\rho^{2}+L^{2}} \mathrm{~d} \Omega_{3}{ }^{2}$,

where $\rho^{2}=r^{2}-L^{2}$ and $\Omega_{3}$ are spherical coordinates in the 4567-space. For $\rho \rightarrow \infty$, this is the metric of $A d S_{5} \times S^{3}$. When $\rho=0$ (i.e., $r^{2}=L^{2}$ ), the radius of the $S^{3}$ shrinks to zero.

The scalar mode with dimension $\Delta=3$ (i.e., supergravity mass $\left.M_{\text {sugra }}^{2}=\Delta(\Delta-4)=-3\right)$ maps to the fermion bilinear $\tilde{\psi} \psi$ in the dual field theory. This mode corresponds to an imaginary AdS mass. However, this mass is above the Breitenlohner-Freedman bound [78, 79] for $A d S_{5}\left(M_{B F}^{2}=-4\right)$ and thus guarantees stability. For this it is important that the D7 branes do not carry any net charge from the five-dimensional point of view, since they wrap a topologically trivial cycle with zero flux.

\subsection{D7 brane fluctuations and mesons in $\mathcal{N}=2$ theory}

The fluctuations of the D7 brane give rise to the mesons and we can determine their masses. This is similar to previously studied supergravity fluctuations which give rise to glueball masses [74].

\subsubsection{Scalar field fluctuations (spin 0)}

As an example, we discuss the fluctuation modes and meson masses for the scalar fields, following the discussion of [17].

The directions transverse to the D7 brane are chosen to be $w^{5}$ and $w^{6}$, and the embedding is as follows:

$$
w_{5}=0+\delta w_{5}, \quad w_{6}=L+\delta w_{6},
$$




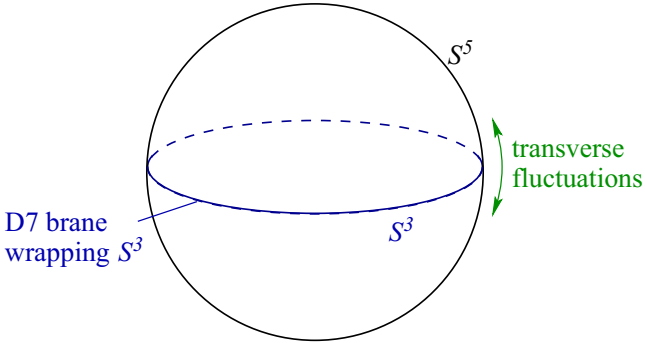

Fig. 5. Fluctuations of the $S^{3}$ wrapped by the D7 probe inside $S^{5}$. These modes give rise to the meson masses.

where $\delta w_{5}$ and $\delta w_{6}$ are the transverse scalar fluctuations shown in fig. 5. To calculate the spectra of the worldvolume fields it is sufficient to work to quadratic order. For the scalars, the relevant part of the Lagrangian density is

$$
\mathcal{L} \simeq-\mu_{7} \sqrt{-\operatorname{det} g_{a b}}\left(1+\frac{1}{2} \frac{R^{2}}{r^{2}} g^{c d} \partial_{c} \Phi \partial_{d} \Phi\right) .
$$

Here, $\Phi$ is used to denote either (real) fluctuation, $\delta w_{5,6}$, and $g_{a b}$ is the induced metric on the D7 worldvolume as given by (3.8). In spherical coordinates with $r^{2}=\rho^{2}+L^{2}$ the equation of motion becomes

$$
\partial_{a}\left(\frac{\rho^{3} \epsilon_{3}}{\rho^{2}+L^{2}} g^{a b} \partial_{b} \Phi\right)=0 .
$$

$\epsilon_{3}$ is the metric on the unit sphere spanned by $\left(\rho, \Omega_{3}\right)$.

The equation of motion can be expanded as

$$
\frac{R^{4}}{\left(\rho^{2}+L^{2}\right)^{2}} \partial^{\mu} \partial_{\mu} \Phi+\frac{1}{\rho^{3}} \partial_{\rho}\left(\rho^{3} \partial_{\rho} \Phi\right)+\frac{1}{\rho^{2}} \nabla^{i} \nabla_{i} \Phi=0,
$$

where $\nabla_{i}$ is the covariant derivative on the three-sphere. Using separation of variables, an ansatz for the modes may be written as

$$
\Phi=\phi(\rho) e^{i k \cdot x} \mathcal{Y}^{\ell}\left(S^{3}\right),
$$

where $\mathcal{Y}^{\ell}\left(S^{3}\right)$ are the scalar spherical harmonics on $S^{3}$, which satisfy

$$
\nabla^{i} \nabla_{i} \mathcal{Y}^{\ell}=-\ell(\ell+2) \mathcal{Y}^{\ell}
$$

The meson masses are defined by $M^{2}=-k^{2}$ for the wave vector $k$ introduced in (3.13).

Then eq. (3.12) gives rise to an equation for $\phi(\rho)$ that, with the redefinitions

$$
\varrho=\frac{\rho}{L}, \quad \bar{M}^{2}=-\frac{k^{2} R^{4}}{L^{2}},
$$

becomes

$$
\partial_{\varrho}^{2} \phi+\frac{3}{\varrho} \partial_{\varrho} \phi+\left(\frac{\bar{M}^{2}}{\left(1+\varrho^{2}\right)^{2}}-\frac{\ell(\ell+2)}{\varrho^{2}}\right) \phi=0 .
$$

This equation may be solved in terms of a hypergeometric function. Imposing normalizability, the solution is

$\phi(\rho)=\frac{\rho^{\ell}}{\left(\rho^{2}+L^{2}\right)^{n+\ell+1}} F\left(-(n+\ell+1),-n ; \ell+2 ;-\rho^{2} / L^{2}\right)$ with

$$
\bar{M}^{2}=4(n+\ell+1)(n+\ell+2) .
$$

Using this, and $M^{2}=-k^{2}=\bar{M}^{2} L^{2} / R^{4}$, the fourdimensional mass spectrum of scalar mesons is given by

$$
M_{s}(n, \ell)=\frac{2 L}{R^{2}} \sqrt{(n+\ell+1)(n+\ell+2)} .
$$

Normalizability of the modes results in a discrete spectrum with a mass scale set by $L$, the position of the D7 brane.

\subsubsection{Fermionic fluctuations (spin $\frac{1}{2}$ )}

The spectrum of fermionic fluctuations of the D7 brane has been studied in $[80,81]$. These fluctuations are dual to so-called "mesino" operators which are the fermionic superpartners of the mesons. Typical mesino operators with conformal dimension $\Delta=\frac{5}{2}$ and $\Delta=\frac{9}{2}$ are $\mathcal{F} \sim \bar{\psi} q$ and $\mathcal{G} \sim \bar{\psi} \lambda \psi$, where $\psi(q)$ is a quark (squark) and $\lambda$ an adjoint fermion. The precise form of these operators is given in sect. 3.3.4.

The dual fluctuations have spin $\frac{1}{2}$ and are described by the fermionic part of the D7 brane action, that is the supersymmetric completion of the Dirac-Born-Infeld action. This action is given in an explicit form by [82]

$$
\begin{aligned}
S_{\mathrm{D} 7}^{f}= & \frac{\tau_{\mathrm{D} 7}}{2} \int \mathrm{d}^{8} \xi \sqrt{-\operatorname{det} g} \bar{\Psi} \mathcal{P}_{-} \Gamma^{A} \\
& \times\left(D_{A}+\frac{1}{8} \frac{i}{2 \cdot 5 !} F_{N P Q R S} \Gamma^{N P Q R S} \Gamma_{A}\right) \Psi .
\end{aligned}
$$

Here $\xi^{A}$ are the worldvolume coordinates $(A=0, \ldots, 7)$ which, in static gauge, will be identified with the spacetime coordinates $t, x^{1}, \ldots, x^{7}$. The field $\Psi$ is the tendimensional positive chirality Majorana-Weyl spinor of type-IIB string theory and $\Gamma_{A}$ is the pullback of the tendimensional gamma matrix $\Gamma_{M}(M, N, \ldots=0, \ldots, 9)$, $\Gamma_{A}=\Gamma_{M} \partial_{A} x^{M}$. The integration goes over the worldvolume of the D7 brane which wraps a submanifold of $A d S_{5} \times S^{5}$ which asymptotes to $A d S_{5} \times S^{3}$. The spinor $\Psi=\Psi\left(x^{M}, \theta^{m}\right)$ depends on the coordinates $x^{M}$ of $A d S_{5}$ and the three angles $\theta^{m}=\left(\theta^{1}, \theta^{2}, \theta^{3}\right)$ of the three-sphere $S^{3}$. The operator $\mathcal{P}_{-}$is a $\kappa$-symmetry projector ensuring $\kappa$-symmetry invariance of the action. The action $S_{\mathrm{D} 7}=$ $S_{\mathrm{D} 7}^{b}+S_{\mathrm{D} 7}^{f}$ with $S_{\mathrm{D} 7}^{b}$ and $S_{\mathrm{D} 7}^{f}$ given by (3.3) and (3.20) is therefore invariant under supersymmetries corresponding to any bulk Killing spinor.

We must now evaluate the five-form $F_{N P Q R S}$ as well as the curved-space-time covariant derivative $D_{M}$ on $A d S_{5} \times$ $S^{5}[80,81]$. This will give a Dirac-type equation which will then be transformed into a second-order differential equation. The fluctuations are assumed to be of planewave type, $\Psi(x, \rho)=\psi_{\ell, \pm}(\rho) e^{i k_{\mu} x^{\mu}} \chi_{\ell}^{ \pm}$, where $\psi_{\ell, \pm}$ and $\chi_{\ell}^{ \pm}$are spinors on $A d S_{5}$ and $S^{5}$, respectively ${ }^{4} \cdot M^{2}=-k^{2}$

\footnotetext{
4 The \pm signs refer to the eigenvalues of the spinor spherical
} harmonics on $S^{3}, \lambda R= \pm\left(\ell+\frac{3}{2}\right)$. 
is again interpreted as the mass of the dual operator. After a somewhat lengthy calculation, which we do not present here, one obtains $[80]^{5}$

$$
\begin{aligned}
& {\left[\partial_{\rho}^{2}+\frac{3}{\rho}\left(1+\frac{\rho^{2}}{r^{2}}\right) \partial_{\rho}+\frac{M^{2} R^{4}}{r^{4}}-\frac{3}{4} \frac{\rho^{2}}{r^{4}}\right.} \\
& +\frac{1}{r^{2}}\left(7-2 m_{\ell} R+\left(2 m_{\ell} R-1\right) \gamma^{\rho}\right) \\
& \left.-\frac{1}{\rho^{2}}\left(\left(m_{\ell} R-1\right)^{2}-\frac{3}{4}+\left(m_{\ell} R-1\right) \gamma^{\rho}\right)\right] \psi_{\ell, \pm}(\rho)=0 \\
& \quad(\ell \geq 0),
\end{aligned}
$$

where $r^{2}=\rho^{2}+L^{2}$, and the distance $L$ is proportional to the quark mass $m_{q}, L=2 \pi \alpha^{\prime} m_{q} . m_{\ell} R$ represents one of the masses

$$
m_{\ell,+} R=\frac{5}{2}+\ell, \quad m_{\ell,-} R=-\left(\frac{1}{2}+\ell\right) .
$$

The spin- $\frac{1}{2}$ operators dual to the fluctuations $\psi_{\ell, \pm}$ will be denoted by $\mathcal{G}_{\alpha}^{\ell}$ and $\mathcal{F}_{\alpha}^{\ell}$. The mass-dimension relation for spin- $\frac{1}{2}$ fields, $|m|=\Delta-2$, determines the conformal dimensions of these operators:

$$
\Delta_{\mathcal{G}}=\frac{9}{2}+\ell, \quad \Delta_{\mathcal{F}}=\frac{5}{2}+\ell \quad(\ell \geq 0) .
$$

We must also ensure that the operators $\mathcal{G}_{\alpha}^{\ell}$ and $\mathcal{F}_{\alpha}^{\ell}$ have the same $S O(4)$ and $U(1)_{R}$ quantum numbers as the fluctuations. For instance, the spinorial spherical harmonics on $S^{3}$ transform in the $\left(\frac{\ell+1}{2}, \frac{\ell}{2}\right)$ and $\left(\frac{\ell}{2}, \frac{\ell+1}{2}\right)$ of $S O(4)=$ $S U(2) \times S U(2)$, while the $U(1)_{R}$ charge is +1 . These properties uniquely fix the structure of $\mathcal{G}_{\alpha}^{\ell}$ and $\mathcal{F}_{\alpha}^{\ell}$. Their explicit form is given in sect. 3.3.4.

The fluctuation equation (3.21) can now be solved in terms of hypergeometric functions. For instance, for the fluctuations $\psi_{\ell,+}$ the solution is given by [80]

$$
\begin{aligned}
\psi_{\ell,+}= & u_{+} \varrho^{\ell+1}\left(1+\varrho^{2}\right)^{-\frac{1}{2}\left(2 \lambda+\frac{3}{2}\right)} \\
& \times F\left(-\lambda, \ell-\lambda+2, \ell+3,-\varrho^{2}\right) \\
& +u_{-} \varrho^{\ell}\left(1+\varrho^{2}\right)^{-\frac{1}{2}\left(2 \lambda+\frac{3}{2}\right)} \\
& \times F\left(-\lambda-1, \ell-\lambda+2, \ell+2,-\varrho^{2}\right),
\end{aligned}
$$

where we rescaled $\varrho=\frac{\rho}{L}, \bar{M}^{2}=\frac{M^{2} R^{4}}{L^{2}}$, and defined $\lambda$ by $\bar{M}^{2}=4 \lambda(\lambda+1)$. The spinors satisfy $\gamma^{\rho} u_{ \pm}= \pm u_{ \pm}$and $u_{-}=\frac{\gamma^{\mu} k_{\mu}}{k^{2}} u_{+}$.

In order for the solution to be well behaved at large radii, $\varrho \rightarrow \infty$, the solution is subjected to the quantization condition

$$
-n=\ell-\lambda+2 .
$$

Solving this for $\bar{M}^{2}$, we obtain the fluctuation masses

$$
\bar{M}_{\mathcal{G}}^{2}=4(n+\ell+2)(n+\ell+3),
$$

which is the spectrum of the operators $\mathcal{G}_{\alpha}^{\ell}$. The spectrum of $\mathcal{F}_{\alpha}^{\ell}$ is obtained in a similar way by solving the equations of motion for $\psi_{\ell,-}$.

\footnotetext{
${ }^{5}$ For overlapping D3/D7 branes $(L=0)$ this equation reduces to that found in [81].
}

\subsubsection{Gauge field fluctuations (spin 1)}

The fluctuations of the D7 worldvolume gauge field $A_{M}$ $(M=0, \ldots, 7)$ give rise to three further mass spectra denoted by $M_{I, \pm}, M_{I I}$ and $M_{I I I}$ in [17]. These spectra are generated by plane-wave fluctuations of the components $A_{i}$ (along the $S^{3}$ ), $A_{\mu}$ (along $x^{0, \ldots, 3}$ ) and $A_{\rho}$ (along the radial direction $\rho$ ) of the eight-dimensional worldvolume gauge field $A_{M}=\left(A_{\mu}, A_{\rho}, A_{i}\right)$. Details on the computation of these spectra can be found in [17].

\subsubsection{Fluctuation-operator matching}

So far we discussed the mass spectra of open string fluctuations on the D7 branes. In order to interpret these spectra as those of meson-like operators, we must map the fluctuations to the corresponding meson operators in the dual field theory. In the following we construct these operators and assign them to the corresponding open string fluctuations.

As was first found in [17], the complete set of D7 brane fluctuations fits into a series of massive gauge supermultiplets of the $\mathcal{N}=2$ supersymmetry algebra. These multiplets contain $16(\ell+1)$ states with the masses

$$
M^{2}=\frac{4 L^{2}}{R^{4}}(n+\ell+1)(n+\ell+2) \quad(n, \ell \geqslant 0) .
$$

Since the supercharges commute with the generators of the global group $S U(2)_{\Phi}$, the $S U(2)_{\Phi}$ quantum number $\frac{\ell}{2}$ is the same for all fluctuations in a supermultiplet.

All D7 brane fluctuations and their quantum numbers are listed in table 3 . The notation of the fluctuations and their mass spectra is the same as in [17]. The numbers $\left(j_{\Phi}, j_{\mathcal{R}}\right)_{q}$ label a representation of $S O(4) \approx$ $S U(2)_{\Phi} \times S U(2)_{\mathcal{R}}$, and $q$ is the $U(1)_{R}$ charge. In order to count the number of states in a multiplet we must take into account the degeneracy in the $S U(2)_{\mathcal{R}}$ quantum number, i.e. we count the degrees of freedom of a particular massive fluctuation and multiply it with $\left(2 j_{\mathcal{R}}+1\right)$. Then, the number of bosonic components in a multiplet is

$1\left(2\left(\frac{\ell}{2}+1\right)+1\right)+(2+3+1)\left(2 \frac{\ell}{2}+1\right)+1\left(2\left(\frac{\ell}{2}-1\right)+1\right)=8(\ell+1)$.

Of course, this agrees with the number of fermionic components,

$$
4\left(2 \frac{\ell+1}{2}+1\right)+4\left(2 \frac{\ell-1}{2}+1\right)=8(\ell+1),
$$

giving altogether $16(\ell+1)$ states.

We now assign operators to the D7 brane fluctuations appearing in table 3 . Note that the masses are above the Breitenlohner-Freedman bound [78,79] and thus admissible, even if their square is negative. Open strings are dual to composite operators with fundamental fields at their ends: scalars $q^{m}=(q, \overline{\tilde{q}})^{T}$ and spinors $\psi_{i}=\left(\psi, \tilde{\psi}^{\dagger}\right)^{T}$. We will refer to these operators as mesons and their superpartners as mesinos. We must ensure that the operators have the same quantum numbers (i.e., spin, global symmetries, etc.) as the corresponding fluctuations. Also, the 
Table 3. Field content of the $\mathcal{N}=2$ supermultiplets in the D3/D7 theory.

\begin{tabular}{|c|c|c|c|c|cc|c|c|}
\hline & Fluctuation & d.o.f. & $\left(j_{\Phi}, j_{\mathcal{R}}\right)_{q}$ & 5 d mass & Spectrum & Op. & $\Delta$ \\
\hline Mesons & 1 scalar & 1 & $\left(\frac{\ell}{2}, \frac{\ell}{2}+1\right)_{0}$ & $m^{2}=-4$ & $M_{I,-}(n, \ell+1)$ & $(\ell \geq 0)$ & $\mathcal{C}^{I \ell}$ & 2 \\
(bosons) & 2 scalars & 2 & $\left(\frac{\ell}{2}, \frac{\ell}{2}\right)_{2}$ & $m^{2}=-3$ & $M_{s}(n, \ell)$ & $(\ell \geq 0)$ & $\mathcal{M}_{s}^{A \ell}$ & 3 \\
& 1 scalar & 1 & $\left(\frac{\ell}{2}, \frac{\ell}{2}\right)_{0}$ & $m^{2}=-3$ & $M_{I I I}(n, \ell)$ & $(\ell \geq 1)$ & $\mathcal{J}^{5 \ell}$ & 3 \\
& 1 vector & 3 & $\left(\frac{\ell}{2}, \frac{\ell}{2}\right)_{0}$ & $m^{2}=0$ & $M_{I I}(n, \ell)$ & $(\ell \geq 0)$ & $\mathcal{J}^{\mu \ell}$ & 3 \\
& 1 scalar & 1 & $\left(\frac{\ell}{2}, \frac{\ell}{2}-1\right)_{0}$ & $m^{2}=0$ & $M_{I,+}(n, \ell-1)$ & $(\ell \geq 2)$ & - & 4 \\
\hline Mesinos & 1 Dirac & 4 & $\left(\frac{\ell}{2}, \frac{\ell+1}{2}\right)_{1}$ & $|m|=\frac{1}{2}$ & $M_{\mathcal{F}}(n, \ell)$ & $(\ell \geq 0)$ & $\mathcal{F}_{\alpha}^{\ell}$ & $\frac{5}{2}$ \\
(fermions) & 1 Dirac & 4 & $\left(\frac{\ell}{2}, \frac{\ell-1}{2}\right)_{1}$ & $|m|=\frac{5}{2}$ & $M_{\mathcal{G}}(n, \ell-1)$ & $(\ell \geq 1)$ & $\mathcal{G}_{\alpha}^{\ell}$ & $\frac{9}{2}$ \\
\hline
\end{tabular}

five-dimensional mass of a fluctuation and the conformal dimension of the dual operator must satisfy a particular relation depending on the spin, e.g., $m^{2}=\Delta(\Delta-4)$ for scalars.

Let us construct gauge invariant operators for the bosonic fluctuations $[17,75,81]$. First, there is a scalar in the $\left(\frac{\ell}{2}, \frac{\ell}{2}+1\right)_{0}$ with 5 d mass $m^{2}=-4+\ell \geq m_{B F}^{2}{ }^{6}$ which corresponds to the $\Delta=\ell+2$ chiral primaries

$$
\mathcal{C}^{I \ell}=\bar{q}^{m} \sigma_{m n}^{I} X^{\ell} q^{n} .
$$

Here the Pauli matrices $\sigma_{m n}^{I}(I=1,2,3)$ transform in the triplet representation of $S U(2)_{R}$, while $q^{m}, \psi^{i}$ and $X^{\ell}$ have the $S O(4)$ quantum numbers $\left(0, \frac{1}{2}\right),(0,0)$ and $\left(\frac{\ell}{2}, \frac{\ell}{2}\right)$, respectively. $X^{\ell}$ denotes the symmetric, traceless operator insertion $X^{\left\{i_{1}\right.} \cdots X^{\left.i_{\ell}\right\}}$ of $\ell$ adjoint scalars $X^{i}(i=$ $4,5,6,7)$. This operator insertion generates operators with higher angular momentum $\ell$.

Then, there are 2 scalars in the $\left(\frac{\ell}{2}, \frac{\ell}{2}\right)_{2}$ which we are dual to the scalar meson operators

$$
\mathcal{M}_{s}^{A \ell}=\bar{\psi}_{i} \sigma_{i j}^{A} X^{\ell} \psi_{j}+\bar{q}^{m} X_{V}^{A} X^{\ell} q^{m} \quad(i, m=1,2)
$$

which have conformal dimensions $\Delta=\ell+3$. Here $X_{V}^{A}$ denotes the vector $\left(X^{8}, X^{9}\right)$ and $\sigma^{A}=\left(\sigma^{1}, \sigma^{2}\right)$ is a doublet of Pauli matrices. Both $X_{V}^{A}$ and $\sigma^{A}$ transform in the $\mathbf{2}$ of $U(1)_{R}$. The operators $\mathcal{M}_{s}^{A \ell}$ thus transform in the $\left(\frac{\ell}{2}, \frac{\ell}{2}\right)$ of $S O(4)$ and are charged +2 under $U(1)_{R}$.

Next, there is a vector in the $\left(\frac{\ell}{2}, \frac{\ell}{2}\right)_{0}$ associated with the $\Delta=\ell+3$ operator

$$
\begin{gathered}
\mathcal{J}^{\mu \ell}=\bar{\psi}_{i}^{\alpha} \gamma_{\alpha \beta}^{\mu} X^{\ell} \psi_{i}^{\beta}+i \bar{q}^{m} X^{\ell} D^{\mu} q^{m}-i \bar{D}^{\mu} \bar{q}^{m} X^{\ell} q^{m} \\
(\mu=0,1,2,3)
\end{gathered}
$$

which we identify as the $U\left(N_{f}\right)$ flavour current.

Finally, there is a (pseudo-)scalar in the $\left(\frac{\ell}{2}, \frac{\ell}{2}\right)_{0}$ dual to $\mathcal{J}^{5 \ell-1}=\bar{\psi}_{i}^{\alpha} \gamma_{\alpha \beta}^{5} X^{\ell-1} \psi_{i}^{\beta}+\ldots(\ell \geq 1)$ and a scalar in the $\left(\frac{\ell}{2}, \frac{\ell}{2}+1\right)_{0}(\ell \geq 2)$ which corresponds to a higher descendant of $\mathcal{C}^{I \ell}$. These operators do not appear in the lowest $(\ell=0)$ multiplet.

We now turn to the fermionic fluctuations [81]. These fluctuations are dual to so-called mesino operators, the

\footnotetext{
6 The lowest fluctuation has negative mass squared, $m^{2}=$ -4 , saturating the Breitenlohner-Freedman bound, $m_{B F}^{2}=$ $-d^{2} / 4=-4(d=4)$.
}

superpartners of the meson-like operators studied above. There are two types of spin- $\frac{1}{2}$ fluctuations with quantum numbers $\left(\frac{\ell}{2}, \frac{\ell+1}{2}\right)_{1}$ and $\left(\frac{\ell}{2}, \frac{\ell-1}{2}\right)_{1}$. These correspond to the mesino operators

$$
\begin{aligned}
\mathcal{F}_{\alpha}^{\ell}= & \bar{q} X^{\ell} \tilde{\psi}_{\alpha}^{\dagger}+\tilde{\psi}_{\alpha} X^{\ell} q \\
\mathcal{G}_{\alpha}^{\ell-1}= & \bar{\psi}_{i} \sigma_{i j}^{B} \lambda_{\alpha C} X^{\ell-1} \psi_{j}+\bar{q}^{m} X_{V}^{B} \lambda_{\alpha C} X^{\ell-1} q^{m}, \\
& (A, B, C=1,2)
\end{aligned}
$$

which have the conformal dimensions $\Delta=\frac{5}{2}+\ell(\ell \geq 0)$ and $\Delta=\frac{7}{2}+\ell(\ell \geq 1)$, respectively. As their bosonic partners, mesinos have fundamental fields at their ends. The spinors $\lambda_{\alpha A}(A=1,2)$ have the $S O(4)$ quantum numbers $\left(\frac{1}{2}, 0\right)$ and belong to the adjoint hypermultiplets $\left(\Phi_{1}, \Phi_{2}\right)$.

\subsubsection{Interactions}

Form factors for the interactions between the mesons can be computed from higher-order terms in the DBI action. For example, if we consider $N_{f} \mathrm{D} 7$ branes then there are DBI terms of the form

$$
S \sim \int \mathrm{d}^{8} x \sqrt{-g} g^{a b} g^{\mu \nu} f^{a b c} A_{\mu}^{a} A_{\alpha}^{b} \partial_{\nu} A_{\beta}^{c},
$$

where $f^{a b c}$ is a structure constant for the flavour group. $A_{\alpha}$ are the scalar fields discussed above and $A_{\mu}$ describe the vector mesons. If we substitute in the solutions for the meson mass eigenstates we have found above and integrate over the four directions of the D7 transverse to the D3 we are left with the effective interaction between two scalars and a vector meson. Equally, one could replace $A_{\mu}$ by its non-normalizable solution giving the coupling of the two scalars to a flavour gauge boson.

These form factors are explicitly computed for the $\mathcal{N}=2$ theory in [75] (see also [83]). There the form factors are also Fourier transformed to position space to give an estimate of the effective size of the mesons. The typical dimension is given by the inverse of the meson's mass $\sqrt{g_{Y M}^{2} N} / m_{q}[75,84]$.

\subsubsection{Mesons on the Coulomb branch}

The $\mathcal{N}=4$ gauge theory has a large moduli space on which the six adjoint scalars have mutually commuting 
vacuum expectation values. This corresponds in the gravity dual to separating the D3 branes in the six transverse directions to their world volume as discussed above in sect. 2.4 - the gravity dual is a multi-centre solution (2.17). D7 brane probes continue to lie flat in these geometries since the $H$ factors of the metric cancel from the DBI action. The adjoint vev should generate a quark mass through the Yukawa term in the superpotential $\tilde{Q} A Q$. Mesonic fluctuations for some sample geometries have been computed in [85] and indeed for massless quarks the mesons have masses proportional to the vacuum expectation value of the scalars.

\subsection{Holographic heavy-light mesons}

We have seen that meson states made of a quark and its antiquark are described by the open string modes on the surface of a D7 brane in a D3 brane background. We can introduce two quarks with different masses by including two D7 branes with different separations (in the $w_{5}$ or $w_{6}$ directions) from the D3 branes as shown in fig. 6. The strings stretched between the two D7 branes carry the flavour quantum numbers of each of the two branes and therefore they have the correct symmetries to holographically describe the heavy-light meson operators.

In AdS the preferred static configuration for these strings is to lie stretched straight between the D7 branes at constant $\rho$ as if they were in flat space [87]. This can be easily seen from the Nambu-Goto action of the strings,

$$
S_{\text {string }}=T \int \mathrm{d}^{2} \sigma \sqrt{\operatorname{det} G_{M N} \frac{\mathrm{d} X^{M}}{\mathrm{~d} \sigma^{a}} \frac{\mathrm{d} X^{N}}{\mathrm{~d} \sigma^{b}}} .
$$

For a static string the determinant is given by the product of the $G_{t t}$ and $G_{w_{5} w_{5}}$ metric components. In AdS this product cancels to give unity and the action is that in flat space with the straight string minimizing the action. For the moment we will concentrate on this configuration and assume we can give the string some small centre of mass motion without it bending.

As the separation between the two D7 branes is increased the $77^{\prime}$ strings grow and naively one expects these states to have a mass given by the product of their length and tension. This immediately provides an apparent confusion - the mass of the supergravity state is holographically related to the dimension of the operator it is describing in the field theory. Why should the bi-fermionic heavy-light operator's dimension be changing as we increase one quark's mass? To resolve this confusion it is helpful to look at the Polyakov form of the action for the string.

We use the gauge-fixed Polyakov string action

$$
S_{P}=-\frac{T}{2} \int \mathrm{d} \sigma \mathrm{d} \tau G_{\mu \nu}\left(-\dot{X}^{\mu} \dot{X}^{\nu}+X^{\prime \mu} X^{\nu}\right),
$$

so we must also impose the constraint equations

$$
G_{\mu \nu} \dot{X}^{\mu} X^{\prime \nu}=0, \quad G_{\mu \nu}\left(\dot{X}^{\mu} \dot{X}^{\nu}+X^{\prime \mu} X^{\prime \nu}\right)=0 .
$$

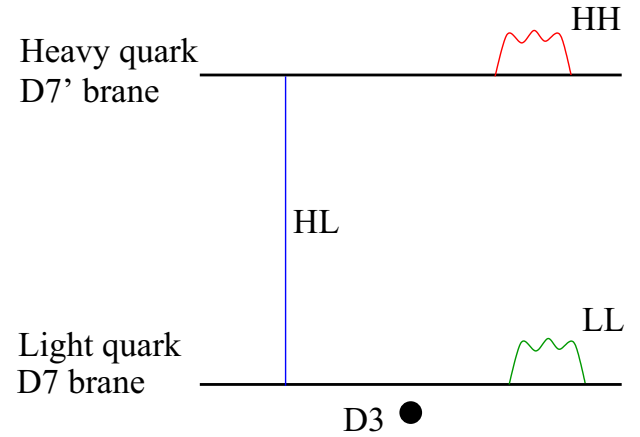

Fig. 6. The brane configuration including both a heavy and a light quark. The 77 and $7^{\prime} 7^{\prime}$ strings are holographically dual to light-light and heavy-heavy mesons, respectively. Heavy-light mesons are holographically described by strings between the two D7 branes - we work in the semi-classical limit where those strings are stretched tight. From [86].

For the configuration we are considering and for a diagonal metric the first constraint vanishes $\left(\dot{w}_{5}=0\right)$. In flat space the second equation, after integration over $\sigma$ gives the familiar $E^{2}-p^{2}=L^{2} T^{2}$ energy momentum relation for the centre-of-mass motion with $L$ the length of the string.

In AdS the $x_{\|}$and $\rho$ directions are distinct and we must be careful. Integrating the metric over $\sigma$ gives

$$
S_{P}=-\frac{T L}{2} \int \mathrm{d} \tau\left[-\tilde{G}_{x x} \dot{x}^{2}-\tilde{G}_{w w} \dot{w}_{i}^{2}+\tilde{G}_{w w}\right]
$$

where

$$
\begin{aligned}
\tilde{G}_{x x} & =\frac{1}{L} \int_{0}^{L} \mathrm{~d} \sigma G_{x x}=\frac{1}{R^{2}}\left(\rho^{2}+\frac{1}{3} L^{2}\right), \\
\tilde{G}_{w w} & =\frac{1}{L} \int_{0}^{L} \mathrm{~d} \sigma G_{w w}=\frac{R^{2}}{\rho L} \arctan (L / \rho) .
\end{aligned}
$$

These are essentially averages of the metric components along the stretched string's length.

The constraint, when integrated over $\sigma$, gives

$$
\tilde{G}^{x x} p_{x}^{2}+\tilde{G}^{w w} p_{w}^{2}+T^{2} L^{2} \tilde{G}_{w w}=0,
$$

where $p_{x}{ }^{\alpha} \equiv \delta \mathcal{L} / \delta \dot{x}_{\alpha}, p_{w}{ }^{i} \equiv \delta \mathcal{L} / \delta \dot{w}_{i}$. Note that (3.41) is a simple modification of the usual $E^{2}-p^{2}=m^{2}$ with the effective mass depending on the $\rho$ position of the string. If we expand for large $\rho$ we obtain

$$
p_{x}^{2}+\frac{\rho^{4}}{R^{4}} p_{w}^{2}+L^{2} T^{2}=0 .
$$

The form of this equation is transparent in terms of the dilatations in the field theory $-x$ is a length whilst $w$ have energy dimensions. The factor of $\rho^{4}$ is clearly necessary. We can now see that for motion in the $\rho$-direction at large $\rho$ (the UV of the field theory) the string mass is effectively zero no matter the length of the string - the "holographic" mass determining the operator dimensions is zero independent of the string length. On the other hand, for 
motion in the $x$-directions the state has a large mass if the string is long and this will be reflected by the meson mass becoming degenerate with the quark mass at large quark mass. Note this behaviour should be compared with the meson mass made of the heavy quark and its antiquark - that state is lighter with the mass suppressed at large 't Hooft coupling by $\sqrt{\lambda}$.

We have assumed above that the straight string can be boosted from rest in the $\rho$-direction. In fact there are not solutions of this form. We know of no studies of moving strings but presumably the string bends. We will continue to work here in the straight string approximation - this is presumably reasonable for short strings or slow moving strings.

\subsubsection{Semi-classical action for heavy-light states}

The classical analysis above of the heavy-light strings of course misses much of the quantum theory - in particular the unexcited string is the tachyon which is not part of the theory and the lightest state is the spin-one gauge field. What we can learn from (3.39) above is that the centre of mass of the string state has the standard action of a particle in a curved space-time although with metric factors averaged over its length. We expect an action in $10 \mathrm{~d}$ of the form

$$
\begin{aligned}
& S=\frac{1}{(2 \pi)^{9} \alpha^{\prime 5}} \int \mathrm{d}^{10} x \sqrt{-\operatorname{det} \tilde{G}_{10}} e^{-\phi} \\
& \times\left(\frac{-1}{4} \tilde{G}^{M N} \tilde{G}^{K L} F_{M K} F_{L K}+M^{2} \tilde{G}_{M N} A^{M} A^{N}\right) .
\end{aligned}
$$

The ends of these string though are tied to D7 branes so we must T-dualize the action twice - the $A^{8,9}$ components of the gauge field become two scalars, $\phi^{a}$, with action

$$
\begin{aligned}
S= & \frac{1}{(2 \pi)^{9} \alpha^{\prime 5}} \int \mathrm{d}^{2} x \sqrt{-\operatorname{det} \tilde{G}_{8-9}} \int \mathrm{d}^{8} x e^{-\phi} \sqrt{-\operatorname{det} \tilde{G}_{0-7}} \\
& \times\left(\tilde{G}^{m n} \tilde{G}_{w w} \partial_{m} \phi^{a} \partial_{n} \phi^{a}+M^{2} \phi^{a 2}\right) .
\end{aligned}
$$

The two-dimensional integral simply gives an overall factor of $(2 \pi R)^{2}$. One must also re-write the dilaton in terms of the dilaton of the T-dual theory (one equates the string coupling of the two theories as described in $[14,88]$ ) $-e^{-\phi_{9}}=e^{-\phi_{7}} \alpha^{\prime} / R^{2}$. We have

$$
\begin{aligned}
S= & \frac{1}{(2 \pi)^{7} \alpha^{\prime 4}} \int \mathrm{d}^{8} x e^{-\phi} \sqrt{-\operatorname{det} \tilde{G}_{0-7}} \\
& \times\left(\tilde{G}^{m n} \tilde{G}_{w w} \partial_{m} \phi^{a} \partial_{n} \phi^{a}+M^{2} \phi^{a 2}\right) .
\end{aligned}
$$

The kinetic term of this action takes the form of the lowest-order expansion of the DBI action for a D7 brane except with metric factors averaged over the $w_{5}$-direction. In the limit of very small D7 separation the metric factors simply become those on the D7 branes' worldvolumes and these states form part of the non-Abelian DBI action. In

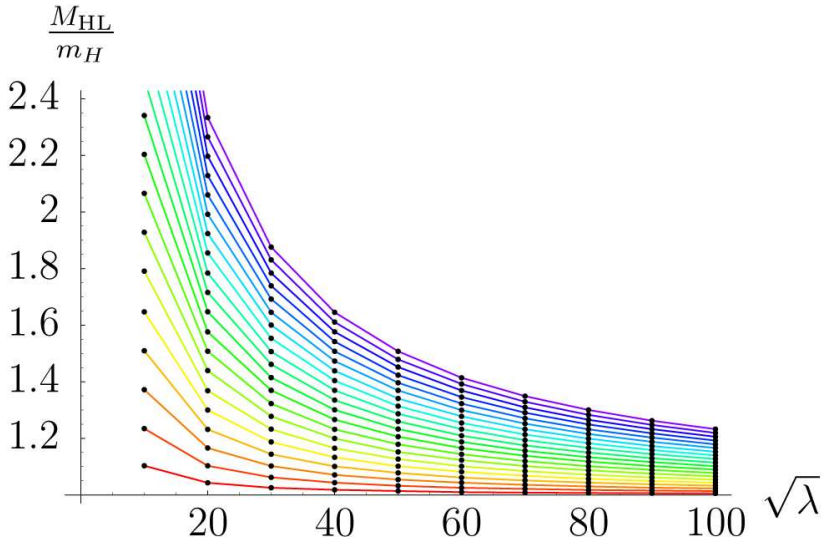

Fig. 7. The masses $M_{H L}$ of the meson and its excited states for the AdS background. The ratio $M_{H L} / m_{H}$, with $m_{H}$ the heavy quark mass (the light quark is taken to be massless), is plotted against the square root of the 't Hooft coupling $\lambda$. We observe that in the large $\lambda$ limit, $M_{H L} / m_{H}$ behaves as $1+$ const $/ \sqrt{\lambda}+\mathcal{O}\left(\lambda^{-1}\right)$. From $[86]$.

addition there is a mass term for the string - in the semiclassical limit of a very long string, one has

$$
M=L T \tilde{G}_{w w}
$$

to be consistent with (3.41).

The heavy-light mesons and their radially excited partners are then described by the holographic equation of motion

$$
\begin{aligned}
& \partial_{\rho} \sqrt{-\operatorname{det} \tilde{G}_{0-7}} \partial_{\rho} \phi+\sqrt{-\operatorname{det} \tilde{G}_{0-7}} \tilde{G}_{w w} \tilde{G}^{x x} \partial_{x}^{2} \phi \\
& -\sqrt{-\operatorname{det} \tilde{G}_{0-7}} \tilde{G}_{w w}^{2} L T \phi=0,
\end{aligned}
$$

with solutions of the form $\phi(x, \rho)=f(r) e^{i k \cdot x},-k^{2}=M^{2}$ as usual. A plot of the solutions from [86] is shown in fig. 7 - the meson masses divided by the heavy-quark mass are plotted as a function of the 't Hooft coupling. Note that at large 't Hooft coupling, the meson mass is just that of the heavy quark (or long string) as expected.

\subsubsection{Heavy-light mesons from non-Abelian DBI action}

A different approach to the holographic description of heavy-light mesons has been proposed in [89]. Again in this case, two D7 brane probes are embedded at different positions into the ten-dimensional gravity background. Now, however, these two branes are described by a non-Abelian Dirac-Born-Infeld action. In this action, the worldvolume fields are assigned to $U\left(N_{f}\right)$ matrix-valued functions for $N_{f} \mathrm{D} 7$ branes. We choose $N_{f}=2$. The embedding configuration of the two D7 branes is determined by the diagonal components of the scalar fields. The corresponding equation of motion is solved by the profile functions of two separated branes, one of which corresponds to the heavy and one to the light quark. The quark masses are given by the boundary values of the two embedded 
branes. The fluctuations of the diagonal elements of the $2 \times 2$ flavour matrices correspond to the light-light and heavy-heavy mesons, respectively. On the other hand, the off-diagonal components of the fluctuations of the fields on the branes are identified with the heavy-light mesons.

\section{Embeddings}

The starting point is the non-Abelian Dirac-Born-Infeld action in curved space proposed by Myers in [88]. This action describes the dynamics of $N_{f} \mathrm{D} p$ branes in a background with metric $G_{m n}$ and is given by

$$
\begin{aligned}
S_{N_{f}}=- & \tau_{p} \int \mathrm{d}^{p+1} \xi e^{-\phi} \\
\times \operatorname{STr}( & \sqrt{-\operatorname{det}\left(P\left[G_{r s}+G_{r a}\left(Q^{-1}-\delta\right)^{a b} G_{s b}\right]+T^{-1} F_{r s}\right)} \\
& \left.\times \sqrt{\operatorname{det} Q^{a} b}\right),
\end{aligned}
$$

where the matrix $Q^{a}{ }_{b}$ is defined by

$$
Q^{a}{ }_{b}=\delta^{a}{ }_{b}+i T\left[X^{a}, X^{c}\right] G_{c b}, \quad T^{-1}=2 \pi \alpha^{\prime},
$$

and $X^{a}$ are the coordinates transverse to the stack of branes, which now take values in a $U\left(N_{f}\right)$ algebra. The symbol STr denotes the symmetrized trace $\operatorname{STr}\left(A_{1}, \ldots, A_{n}\right) \equiv \frac{1}{n !} \operatorname{Tr}\left(A_{1} \ldots A_{n}+\right.$ all permutations $)$ and is needed to avoid the ambiguity of the ordering of the expansion of all fields in the DBI action.

This non-Abelian DBI action is now used to find the embedding of $N_{f}$ probe D7 branes in different gravity backgrounds. The embedding profiles correspond to the classical solutions for the scalar fields in the D7 brane action. In our case, the scalar fields $X^{a}$ are $U\left(N_{f}\right)$ matrixvalued functions, which makes it difficult to obtain a general form of the profile functions. In order to simplify the problem, we use the diagonal ansatz

$$
X^{a}=\operatorname{diag}\left(w_{1}^{a}, \cdots, w_{N_{f}}^{a}\right),
$$

thereby setting all off-diagonal components to zero. Here each of the functions $w_{i}^{a}$ corresponds to one of the $N_{f}$ D7 branes.

The quark mass for each flavour is given by the asymptotic value of $w_{i}^{a}$ in the ultraviolet limit. They are the integration constants and given by hand as parameters of the theory. The equations of motion for the $w_{i}^{a}$ are obtained from the action

$$
\begin{aligned}
S_{N_{f}} & =\tau_{7} \int \mathrm{d}^{8} \xi e^{-\Phi} \operatorname{STr}\left(\sqrt{-\operatorname{det}\left(G_{r s}+G_{a b} \partial_{r} w_{i}^{a} \partial_{s} w_{i}^{b}\right)}\right) \\
& =\tau_{7} \int \mathrm{d}^{8} \xi e^{-\Phi} \sum_{i=1}^{N_{f}} \sqrt{-\operatorname{det}\left(G_{r s}+G_{a b} \partial_{r} w_{i}^{a} \partial_{s} w_{i}^{b}\right)},
\end{aligned}
$$

which is eq. (3.48) for the embedding (3.50) and $p=7$. The essential point is here that for the diagonal ansatz (3.50), we obtain $N_{f}$ decoupled equations of motion for the $w_{i}^{a}$, such that the embeddings of each of the probe branes is independent of the other. In other words, for diagonal embeddings the non-Abelian DBI action reduces to the sum of $N_{f}$ Abelian DBI actions.

\section{Fluctuations}

We now consider the scalar and vector meson spectra obtained by considering the fluctuations about the background given. At this stage, we restrict to the case of $N_{f}=2$ flavours or two D7 branes such that the scalar and vector fields in the non-Abelian DBI action are represented by $(2 \times 2)$-matrices. For the classical embedding, we choose the diagonal configuration given by

$$
\bar{X}^{8}=0, \quad \bar{X}^{9}=\left(\begin{array}{cc}
w_{1} & 0 \\
0 & w_{2}
\end{array}\right) .
$$

In terms of the Pauli matrices

$$
\begin{array}{rlr}
\tau^{0} & =\frac{1}{2}\left(\begin{array}{ll}
1 & 0 \\
0 & 1
\end{array}\right), & \tau^{1}=\frac{1}{2}\left(\begin{array}{ll}
0 & 1 \\
1 & 0
\end{array}\right), \\
\tau^{2} & =\frac{1}{2}\left(\begin{array}{rr}
0 & -i \\
i & 0
\end{array}\right), & \tau^{3}=\frac{1}{2}\left(\begin{array}{rr}
1 & 0 \\
0 & -1
\end{array}\right),
\end{array}
$$

$\bar{X}^{9}$ can be rewritten as

$$
\bar{X}^{9}=w \tau_{0}+v \tau_{3}, \quad w_{1}=(w+v) / 2, \quad w_{2}=(w-v) / 2,
$$

where $v=w_{1}-w_{2}$. The asymptotic boundary values of $w_{1}$ and $w_{2}$ correspond to the heavy and light quark masses, respectively. When $v=0$, the two branes are at the same place, $w_{1}=w_{2}=w$, corresponding to a $U(2)$ flavour symmetry. For $v \neq 0$ this flavour symmetry is explicitly broken.

The scalar and gauge field fluctuations are taken to be of the form $(a=8,9)$

$$
\begin{aligned}
X^{9} & =\bar{X}^{9}+\phi^{9}, \quad X^{8}=\phi^{8}, \\
\phi^{a} & =\phi_{0}^{a} \tau^{0}+\phi_{i}^{a} \tau^{i}, \quad A^{r}=A_{0}^{r} \tau^{0}+A_{i}^{r} \tau^{i},
\end{aligned}
$$

and can be written as

$$
\phi^{a}=\left(\begin{array}{cc}
\phi_{+}^{a} & \phi_{12}^{a} \\
\phi_{21}^{a} & \phi_{-}^{a}
\end{array}\right),
$$

similarly $A^{r}$. The diagonal elements $\phi_{ \pm}^{a}=\phi_{0}^{a} \pm \phi_{3}^{a}$ describe fluctuations of each brane and are dual to the heavy-heavy and light-light mesons. On the other hand, the off-diagonal elements $\phi_{12}^{a}=\phi_{1}^{a}-i \phi_{2}^{a}$ and $\phi_{21}^{a}=\phi_{1}^{a}+i \phi_{2}^{a}$ correspond to fluctuations of strings stretched between the two branes and are dual to the heavy-light mesons. The mass of this last type of fluctuations will depend on $v$. A similar structure emerges also for gauge field fluctuations $A_{r}$.

These meson mass spectra are obtained by solving the linearized equation of motions for the field fluctuations. For the $A d S_{5} \times S^{5}$ background, the heavy-light meson masses are obtained from

$$
\begin{aligned}
& \left(\partial_{\rho}^{2}+\frac{3}{\rho} \partial_{\rho}-\frac{l(l+2)}{\rho^{2}}+\frac{M^{2}-v^{2}}{2}\right. \\
& \left.\quad \times\left(\left(\frac{R^{2}}{\rho^{2}+w_{1}^{2}}\right)^{2}+\left(\frac{R^{2}}{\rho^{2}+w_{2}^{2}}\right)^{2}\right)\right) \phi=0 .
\end{aligned}
$$



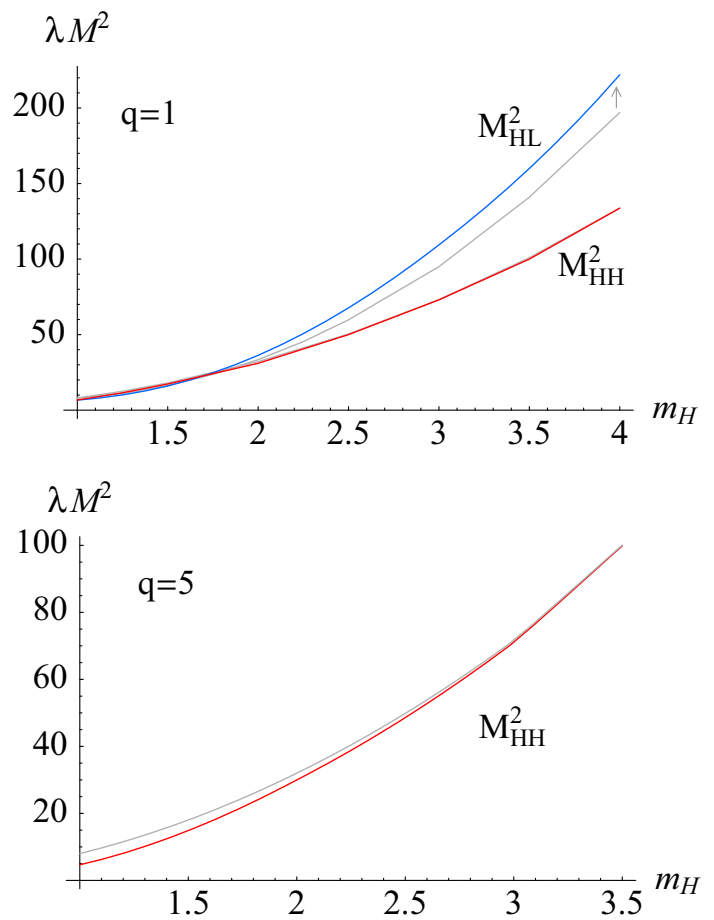

Fig. 8. Meson masses for non-zero $q$. The red and blue curves show $M_{H L}, M_{H H}$ for $\lambda=3^{4}, q=1$ (top) and $q=5$ (bottom). The grey curves show the corresponding meson masses for $q=0$. The presence of $q$ increases the HL meson masses. The lambda dependence remains unchanged. From [89].

For $w_{1}=w_{2}=w$, we get $v=0$ and the equation reduces to the one given by Kruczenski et al. [17] which can be solved analytically, as described in sect. 3.16 above.

A central point is that the $\lambda$-dependence of the heavylight meson mass obtained from the non-Abelian DBI action, as described here, coincides with the one obtained using the Polyakov action approach discussed in sect. 3.4.1 above. A finite contribution to the mass remains in the limit of $\lambda \rightarrow \infty$. This contribution corresponds to the minimum energy of a classical string connecting two separated D7 branes, and thus is equivalent to the mass obtained from the Polyakov action.

In general, (3.58) must be solved numerically. However, for a heavy-light meson with a very heavy quark, $w_{2} \gg$ $w_{1}$, the term in (3.58) involving $w_{2}$ is much smaller than the one involving $w_{1}$ and may be neglected. In this case, the heavy-light meson mass is found to be

$$
M_{H L}^{2}=\frac{16 w_{1}^{2}}{R^{4}}+\frac{v^{2}}{\left(2 \pi \alpha^{\prime}\right)^{2}}=16 \pi \frac{m_{L}^{2}}{\lambda}+\left(m_{H}-m_{L}\right)^{2},
$$

where we reintroduced the string tension $T=1 /\left(2 \pi \alpha^{\prime}\right)$ (which was set to one above) and defined the quark masses $m_{L, H}=w_{1,2} /\left(2 \pi \alpha^{\prime}\right)$ as the distances $w_{1,2}$ in units of $T$. Equation (3.59) implies that the mass of HL mesons has two different contributions. The first term proportional to $\frac{m_{L}^{2}}{\sqrt{\lambda}}$ has the same dependence on the 't Hooft coupling as in the single flavour case [17]. The second term is dominant at large 't Hooft coupling $(\lambda \rightarrow \infty)$, where the mass of the

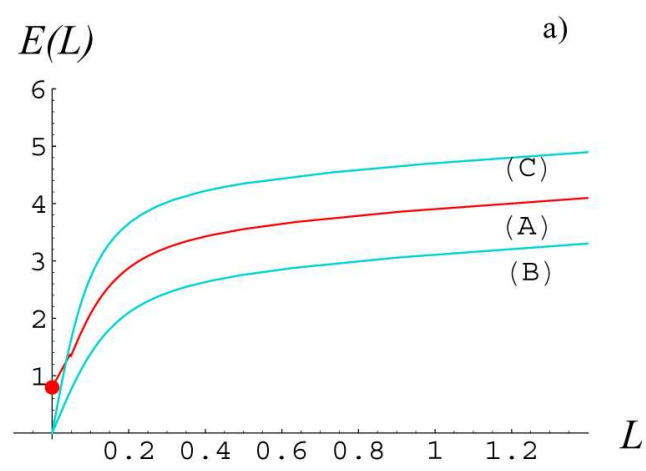

b)

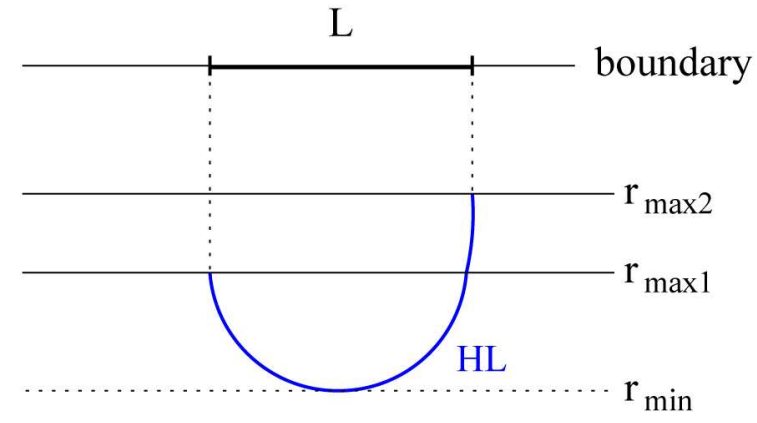

Fig. 9. a) Numerical plots of the energy $E(L)$ for HL mesons (A), LL mesons (B) and HH mesons (C). The circle at the endpoint of the curve (A) shows a finite string energy $E$ at length $L=0$. Here we set $q=5$ and $R=1$, and the brane positions are taken at $r_{\max 1}=10$ and $r_{\max 2}=15$, respectively. b) Schematic plot of the Wilson loop. Figure from [89].

HL mesons is approximated by the second term,

$$
M_{H L} \approx \frac{v}{2 \pi \alpha^{\prime}}=m_{H}-m_{L} .
$$

In this strong-coupling regime, the heavy-light meson mass depends solely on the difference of the two quark masses. This is consistent with the result obtained in [86], discussed in sect. 3.4.1 above.

This $\lambda$-dependence persists if instead of the $A d S_{5} \times S^{5}$ background, we consider a deformed gravity background as introduced in sect. 2.4 above. As an example, we consider the $\mathrm{D} 3+\mathrm{D}(-1)$ gravity background of [90]. This is an example of a dilaton flow background, in which the dilaton has a non-trivial profile. The field theory dual to this background is a confining $\mathcal{N}=1$ supersymmetric theory, in which a condensate $q \equiv \pi^{2}\left\langle F^{2}\right\rangle$ is switched on. The background in string frame is given by a non-trivial dilaton $\Phi$ and axion $\chi[90]$,

$$
\mathrm{d} s_{10}^{2}=e^{\Phi / 2}\left(\frac{r^{2}}{R^{2}} A^{2}(r) \eta_{\mu \nu} \mathrm{d} x^{\mu} \mathrm{d} x^{\nu}+\frac{R^{2}}{r^{2}} \mathrm{~d} r^{2}+R^{2} \mathrm{~d} \Omega_{5}^{2}\right),
$$

where

$$
A=1, \quad e^{\Phi}=1+\frac{q}{r^{4}}, \quad \chi=-e^{-\Phi}+\chi_{0} .
$$


In this case, there is only a $\mathcal{N}=1$ supersymmetry remaining for the background with D7 brane probes embedded. Therefore, vector and scalar mesons are no longer degenerate, as shown in fig. 8. For large heavy quark masses, supersymmetry and thus the meson mass degeneracy are restored.

It is instructive to compare the $\lambda$-dependence of the meson spectra with the $\lambda$-dependence of the tension. For a classical string stretched between the two D7 brane probes, the string tension is independent of $\lambda$, in agreement with the heavy-light meson mass result found both in the Polyakov and in the non-Abelian DBI approach. For heavy-light mesons, this tension contributes to the meson mass even if the distance $L$ between the quark and anti-quark in the four-dimensional boundary space is zero, in which case it contributes $E=m_{H}-m_{L}$ to the Wilson line energy, see fig. 9. For the heavy-heavy and lightlight mesons, the string tension scales as $m_{q}^{2} / \sqrt{\lambda}$ for small $L[17,91]$. At large $L$, when the dual gauge theory is in the quark confinement phase, there is a long-range linear potential for all the mesons considered.

\subsection{Mesons with large spin $(\mathrm{J} \gg 1)$}

So far we discussed mesons with spin 0 and 1 (and mesinos with spin $\frac{1}{2}$ ). The calculation of the spectrum of mesons with higher four-dimensional spin $J$ would require the quantization of open strings on the D7 branes, which is difficult. However, meson operators with large spin have small anomalous dimensions and quantum corrections are negligible [92]. Large spin mesons therefore have a dual description in terms of a classical rotating string. In the following we show how Regge trajectories in the $\mathcal{N}=2$ theory of the D3/D7 system can be computed by means of a semi-classical string computation.

Following [17,92], we consider a classical open string which rotates in an $A d S_{5} \times S^{5}$ background and ends on a probe D7 brane. This string is dual to a meson with large spin $J$ in the $\mathcal{N}=2$ theory located on the D3/D7 intersection. We start from the classical Nambu-Goto action in the form

$$
S=-T_{s} \int \mathrm{d} \tau \mathrm{d} \sigma \sqrt{\left(\dot{X} \cdot X^{\prime}\right)^{2}-\dot{X}^{2} X^{\prime 2}},
$$

where dots and primes denote differentiation with respect to $\tau$ and $\sigma$, respectively. The scalar product is taken using the $A d S_{5} \times S^{5}$ metric. We parameterize the $A d S_{5}$ metric as

$$
\mathrm{d} s^{2}=\frac{R^{2}}{z^{2}}\left(-\mathrm{d} t^{2}+\mathrm{d} u^{2}+u^{2} \mathrm{~d} \varphi^{2}+\mathrm{d} x_{3}^{2}+\mathrm{d} z^{2}\right),
$$

where $u$ and $\varphi$ are the coordinates of the plane of rotation $x^{1}-x^{2}$. The string has length $2 u_{0}$ and stretches from $-u_{0}$ to $+u_{0}$ along the $u$-direction. The end points of the string are attached to a probe D7 brane located a distance $z_{R}$ in the radial direction. An example of a spinning string is shown in fig. 10.

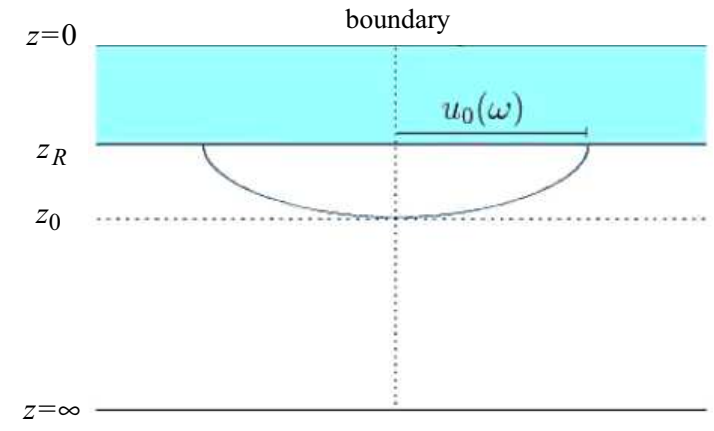

Fig. 10. Example of a string profile $z(u)$.

An appropriate ansatz for a string rotating with constant angular velocity $\omega$ is

$$
t=\tau, \quad \varphi=\omega \tau, \quad u=u(\sigma), \quad z=z(\sigma) .
$$

With this ansatz the Nambu-Goto Lagrangian takes the form $\left(T_{s}=1\right)$

$$
\mathcal{L}=-\frac{R^{2}}{z^{2}} \sqrt{\left(1-\omega^{2} u^{2}\right)\left(u^{\prime 2}+z^{\prime 2}\right)} .
$$

It is convenient to use the rescaled coordinates

$$
\tilde{u}=\omega u, \quad \tilde{z}=\omega z .
$$

In these coordinates, the energy and the angular momentum of the spinning string are given by

$$
\begin{aligned}
& E=\int \mathrm{d} \sigma\left(\omega \frac{\partial \mathcal{L}}{\partial \omega}-\mathcal{L}\right)=\int \mathrm{d} \sigma \frac{\omega}{\mathcal{E}} \frac{R^{2}}{\tilde{z}^{2}} \sqrt{\tilde{u}^{\prime 2}+\tilde{z}^{\prime 2}}, \\
& J=\int \mathrm{d} \sigma \frac{\partial \mathcal{L}}{\partial \omega}=\int \mathrm{d} \sigma \frac{\tilde{u}^{2}}{\mathcal{E}} \frac{R^{2}}{\tilde{z}^{2}} \sqrt{\tilde{u}^{\prime 2}+\tilde{z}^{\prime 2}}
\end{aligned}
$$

where we defined $\mathcal{E}=\sqrt{1-\tilde{u}^{2}}$.

In the gauge $\tilde{u}=\sigma$, we find the following equation of motion for $\tilde{z}(\tilde{u})$ :

$$
\frac{\tilde{z}^{\prime \prime}}{1+\tilde{z}^{\prime 2}}-\frac{\tilde{u}}{\mathcal{E}} \tilde{z}^{\prime}+\frac{2}{\tilde{z}}=0
$$

The solutions of this equation provide the embedding profiles $\tilde{z}(\tilde{u})$ of the spinning string.

Equation (3.70) is a nonlinear differential equation of second order which requires two boundary conditions. These can be obtained from the usual open string boundary terms

$$
\left.\frac{\partial \mathcal{L}}{\partial \tilde{u}^{\prime}} \delta \tilde{u}\right|_{\sigma=0, \pi}=\left.\frac{\partial \mathcal{L}}{\partial \tilde{z}^{\prime}} \delta \tilde{z}\right|_{\sigma=0, \pi}=0
$$

for strings ending on a probe D7 brane at $\tilde{z}=\tilde{z}_{R}=$ const. Such strings have a Neumann boundary condition in the $\tilde{u}$-direction and a Dirichlet boundary condition in the $\tilde{z}$-direction, i.e. $\left.\delta \tilde{u}\right|_{\sigma=0, \pi}$ is arbitrary, whereas $\left.\delta \tilde{z}\right|_{\sigma=0, \pi}=0$. The latter condition holds, if we set $\tilde{z}\left( \pm \tilde{u}_{0}\right)=\tilde{z}_{R}=$ const. The remaining condition $\partial \mathcal{L} /\left.\partial \tilde{u}^{\prime}\right|_{\sigma=0, \pi}=0$ is satisfied, if 


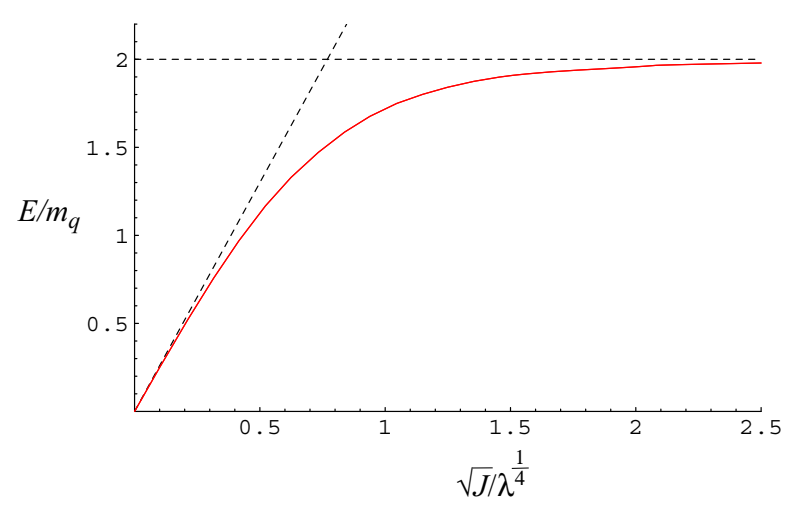

Fig. 11. Numerical Chew-Frautschi plot. The horizontal line (dashed) corresponds to the rest mass of the quark-antiquark pair.

$\left.\tilde{u}^{\prime}\right|_{\sigma=0, \pi}=0$. Using the gauge $\tilde{z}=\sigma$, we see that this corresponds to $\partial \tilde{z} /\left.\partial \tilde{u}\right|_{\tilde{u}= \pm \tilde{u}_{0}} \rightarrow \infty$ which means that the string ends orthogonally on the D7 brane at $\tilde{z}_{R}$. In actual computations of the string profile, the orthogonality condition is inexpedient. We therefore use the fact that the solutions are symmetric around $\tilde{u}=0$, where they have their only maximum, and impose the boundary condition $\tilde{z}^{\prime}(0)=0$.

The Regge trajectories $E(J)$ can now be obtained as follows. We first solve the equation of motion (3.70) for the string profile $\tilde{z}(\tilde{u})$ by integrating $(3.70)$ from $-\tilde{u}_{0}$ to $+\tilde{u}_{0}$. In the shooting technique, we set $\tilde{z}(0)=\tilde{z}_{0}=$ const, $\tilde{z}^{\prime}(0)=0$ such that $\tilde{z}\left( \pm \tilde{R}_{0}\right)=\tilde{z}_{R}$. This yields the string length $u_{0}=\tilde{u}_{0} / \omega$ as the location at which $\tilde{z}^{\prime}\left(\tilde{u}_{0}\right) \rightarrow \infty$. A typical profile is shown in fig. 10. Then, substituting the profiles $\tilde{z}(\tilde{u}) \equiv 0$ into eqs. (3.68) and (3.69), we determine the energy $E(\omega)$ and the angular momentum $J(\omega)$ of the spinning string for various values of the angular velocity $\omega$. The Regge trajectory then corresponds to a curve in the $(\sqrt{J}, E)$-plane parameterized by $\omega$, as shown in fig. 11 .

The $\mathcal{N}=2$ theory we consider is not confining and we therefore expect some deviations from the linear Regge behaviour of QCD. We observe that for small spin $J$ the meson mass approximately follows a linear Regge trajectory, whereas for large $J$ the energy $E(J)$ asymptotes to the rest mass energy. This can be understood from the behaviour of the string length as a function of the spin. At small spin values the length of the string is much smaller than the scale of the space, and the string is effectively rotating in flat space leading to a linear Regge behaviour. At large spin the string is larger than the size of the space. Here the string rotates very slowly and the energy is that of particles moving in a Coulomb potential [17]. The binding energy of the quark-antiquark pair thus vanishes at large spin values and $E(J)$ asymptotes to the rest mass $E=2 m$.

Analysis of high-spin mesons with constituent quarks with different masses can be found in [93]. In these cases with more than one D7 brane at different positions highspin mesons can decay. If the string in fig. 10 dips sufficiently far into the interior of the space that it meets a second D7 brane then the string can split into two seg- ments between the two different branes. The rate for this process has been computed in $[94,95]$.

\subsection{The squark sector from instantons on the D7 probe}

Since the original configuration of [16] is supersymmetric, in addition to the fundamental fermion bilinear there is also a squark (scalar) present in the D3/D7 system. For two coincident D7 branes, the vev of this squark bilinear has been shown to be dual to the radius of an $S U(2)$ instanton on the D7 brane probe [96-98]. The vector meson spectrum for this background has been calculated in [99]. For the part of the Higgs branch dual to a single instanton, the spectrum is computed as a function of the instanton size. It turns out that the zero-size and infinite-size limits are equivalent modulo a singular gauge transformation: In the dual large- $N$ gauge theory, this is an equivalence between the spectrum of the $S U(N)$ theory and the $S U(N-1)$ theory obtained by taking the Higgs vev to infinity. The spectral flow between these limits leads to a non-trivial re-arrangement of the mass eigenstates and global charges. In particular, the flow takes vector mesons in the $(0,0)$ representation of the global $S U(2)_{L} \times S U(2)_{R}$ symmetry, which is unbroken at the origin of moduli space, to vector mesons in the representation $(1,1)$.

For the field theory given by (3.1) with (3.2), on the Higgs branch the vector multiplet moduli $\phi_{3}$ vanish, while $q^{i}$ and $\tilde{q}_{i}$ have non-zero expectation values. Here, the lower-case letters denote the scalar components of the corresponding superfields. There are also mixed CoulombHiggs vacua, for which both $q^{i}, \tilde{q}_{i}$ and $\phi_{3}$ have non-zero expectation values.

For non-zero $m$ and vanishing $\phi_{3}$, the fundamental hypermultiplets are massive and there is no Higgs branch. However, there is a mixed Coulomb-Higgs branch when $\phi_{3}$ has an expectation value such that some components of the hypermultiplets are massless. An example of a point on a mixed Coulomb-Higgs branch is given by a diagonal $\phi_{3}$ for which all but the last $k$ entries on the diagonal are vanishing, with the last $k$ entries equal to $-m$. In this case, the F-flatness equations $\tilde{q}_{i}\left(\phi_{3}+m\right)=\left(\phi_{3}+m\right) q^{i}=0$ permit fundamental hypermultiplet expectation values in which only the last $k$ entries of $q^{i}$ and $\tilde{q}_{i}$ are non-zero.

On the supergravity side, the effective action describing D7 branes in a curved background is given by (3.3). Since we need to consider at least two flavours (two D7s) in order to have a Higgs branch, we have to consider the non-Abelian version of (3.3).

At leading order in $\alpha^{\prime}$, field strengths which are selfdual with respect to the flat four-dimensional metric $\mathrm{d} s^{2}=\sum_{m=1}^{4} \mathrm{~d} y^{m} \mathrm{~d} y^{m}$ solve the corresponding equations of motion, due to a conspiracy between the Chern-Simons and DBI terms. Here the $y^{m}$ denote the 4567-directions wrapped by the D7 brane probe. Inserting the explicit AdS background values for the metric and RamondRamond four-form into the action (3.3), with non-trivial field strengths only in the 4567-directions labelled by $y^{m}$, 
gives

$$
\begin{aligned}
S= & \frac{\mu_{7}\left(2 \pi \alpha^{\prime}\right)^{2}}{4} \int \mathrm{d}^{4} x \mathrm{~d}^{4} y H(r)^{-1} \\
\times & \left(-\frac{1}{2} \epsilon_{m n r s} F_{m n} F_{r s}+F_{m n} F_{m n}\right)= \\
& \frac{\mu_{7}\left(2 \pi \alpha^{\prime}\right)^{2}}{2} \int \mathrm{d}^{4} x \mathrm{~d}^{4} y H(r)^{-1} F_{-}^{2},
\end{aligned}
$$

to leading order in $\alpha^{\prime}$, where $r^{2}=y^{m} y^{m}+\left(2 \pi \alpha^{\prime} m\right)^{2}$ and $F_{m n}^{-}=\frac{1}{2}\left(F_{m n}-\frac{1}{2} \epsilon_{m n r s} F_{r s}\right)$. Field strengths $F_{m n}^{-}=0$, which are self-dual with respect to the flat metric $\mathrm{d} y^{m} \mathrm{~d} y^{m}$, manifestly solve the equations of motion. These solutions correspond to points on the Higgs branch of the dual $\mathcal{N}=2$ theory. Strictly speaking, this is a point on the mixed Coulomb-Higgs branch if $m \neq 0$. In order to neglect the backreaction due to dissolved D3 branes, we are considering a portion of the moduli space for which the instanton number $k$ is fixed in the large- $N$ limit.

In [100], it was found that the instanton is also a solution of the action to all orders in $\alpha^{\prime}$.

The AdS/CFT dictionary for the Higgs branch is obtained by considering the symmetries in both field theory and supergravity as usual. On both sides, for $m \neq 0$ there is a $S O(2,4) \times S U(2)_{L} \times S U(2)_{R} \times U(1)_{R} \times S U(2)_{f}$ symmetry, where $S U(2)_{f}$ stands for the flavour symmetry present if two coincident D7 branes are considered. We focus on that part of the Higgs branch, or mixed Coulomb-Higgs branch, which is dual to a single instanton centered at the origin $y^{m}=0$. The instanton, in "singular gauge," is given by

$$
A_{\mu}=0, \quad A_{m}=\frac{2 \Lambda^{2} \bar{\sigma}_{n m} y_{n}}{y^{2}\left(y^{2}+\Lambda^{2}\right)},
$$

where $\Lambda$ is the instanton size, and

$$
\begin{array}{rlrl}
\bar{\sigma}_{m n} & \equiv \frac{1}{4}\left(\bar{\sigma}_{m} \sigma_{n}-\bar{\sigma}_{n} \sigma_{m}\right), & \sigma_{m} & \equiv\left(i \vec{\tau}, 1_{2 \times 2}\right), \\
\sigma_{m n} \equiv \frac{1}{4}\left(\sigma_{m} \bar{\sigma}_{n}-\sigma_{n} \bar{\sigma}_{m}\right), & \bar{\sigma}_{m} \equiv \sigma_{m}^{\dagger}=\left(-i \vec{\tau}, 1_{2 \times 2}\right) .
\end{array}
$$

with $\vec{\tau}$ being the three Pauli matrices. We choose singular gauge, as opposed to the regular gauge in which $A_{n}=2 \sigma_{m n} y^{m} /\left(y^{2}+\Lambda^{2}\right)$, because of the improved asymptotic behaviour at large $y$. The instanton (3.73) breaks the symmetries to

$$
G=S O(1,3) \times S U(2)_{L} \times \operatorname{diag}\left(S U(2)_{R} \times S U(2)_{f}\right),
$$

and corresponds to a point on the Higgs branch

$$
q_{i \alpha}=v \varepsilon_{i \alpha}, \quad v=\frac{\Lambda}{2 \pi \alpha^{\prime}},
$$

where $q_{i \alpha}$ are scalar components of the fundamental hypermultiplets, labelled by a $S U(2)_{f}$ index $i=1,2$, and a $S U(2)_{R}$ index $\alpha=1,2$. All the broken symmetries are restored in the ultraviolet (large $r$ ), where the theory becomes conformal.

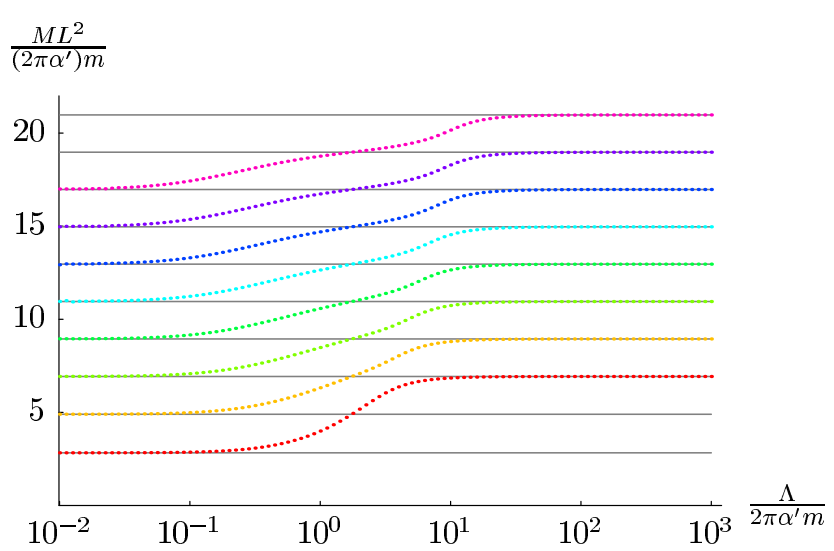

Fig. 12. Meson masses as a function of the Higgs vev, from [99]. Each dotted line represents a regular solution of the equation of motion, corresponding to a vector multiplet of mesons. The vertical axis is $\sqrt{\lambda} M / m$, where $M$ is the meson mass, $\lambda$ the 't Hooft coupling and $m$ the quark mass. The horizontal axis is $v / m$ where $v=\Lambda / 2 \pi \alpha^{\prime}$ is the Higgs VEV. In the limits of zero and infinite instanton size (Higgs vev), one recovers the spectrum (gray horizontal lines) obtained analytically in the absence of an instanton background by [17].

The simplest non-Abelian ansatz for fluctuations $\mathcal{A}_{\mu}$ about the instanton background is given by

$$
\mathcal{A}_{\mu}^{(a)}=\xi_{\mu}(k) f(y) e^{i k_{\mu} x_{\mu}} \tau^{a}, \quad y^{2} \equiv y^{m} y^{m},
$$

which is a singlet under $S U(2)_{L}$ and a triplet under $\operatorname{diag}\left(S U(2)_{R} \times S U(2)_{f}\right) \cdot \tau^{a}$ are the three Pauli matrices. With this ansatz, the vector meson masses are obtained in direct analogy to the method presented in sect. 3.3.1 above. The result for the vector meson masses is shown in fig. 12, where the meson masses in the presence of the instanton are shown as dotted lines, while the full horizontal lines correspond to the meson spectrum without an instanton found in [17], discussed above in sect. 3.3.1. We see that the spectrum is shifted by two levels when moving from zero to infinite instanton size. This may be understood as follows. In singular gauge, the infinite-size instanton is given by

$$
A_{n}=2 \frac{\bar{\sigma}_{m n} y^{m}}{y^{2}} .
$$

By virtue of the singular gauge transformation

$$
U=\sigma^{m} y^{m} /|y|,
$$

$A_{n}$ may be set to zero, which allows for direct comparison with the meson spectrum of [17]. However, performing the same singular gauge transformation on the fluctuations (3.77), we obtain fluctuations in a higher spherical harmonic $\ell=2$ on $S^{3}$. The fact that $\ell=2$ is in exact agreement with the level shift observed in fig. 12 .

The instanton ansatz has also been used for studying the Higgs potential in gravity duals with less supersymmetry $[101,102]$. In particular, it has been used to show with gauge/gravity dual methods that an isospin chemical potential leads to instabilities in supersymmetric theories, in agreement with field theory results [103]. 


\subsection{Summary}

We have introduced quarks into the AdS/CFT correspondence, in the quenched limit $N_{f} \ll N$, by including probe D7 branes. By "quenched" we mean that there are no quark loops contributing to the gauge propagators. The gauge theory resulting from adding D7 probes has $\mathcal{N}=2$ supersymmetry. The D3 and D7 branes can be separated in two directions and the separation gives a mass to the quarks. Fluctuations of the D7 brane in these two directions are dual to scalar and pseudo-scalar mesons. A gauge field on the D7 worldvolume is dual to the vector mesons of the gauge theory.

We have been able to compute a number of meson masses in this strongly coupled supersymmetric model. If the quark mass is zero, the theory is conformal and there are no bound states. When the quark mass, $m_{q}=L / 2 \pi \alpha^{\prime}$, is none zero the masses of mesons made from a single quark flavour are generically given by

$$
M \sim \frac{2 L}{R^{2}} n \sim \frac{2 m_{q} n}{\sqrt{g_{Y M}^{2} N}},
$$

where we have used (2.8) and $n$ is the radial excitation number of the meson.

These mesons are very tightly bound - their mass is suppressed relative to the mass of the quarks they are made of by the 't Hooft coupling which is formally infinite. Note also that these states do not show Regge behaviour $(M \sim \sqrt{n})$. We will discuss the relation of these results to QCD later in sect. 8 on AdS/QCD.

We have also looked at highly spinning strings and strings that are dual to heavy-light mesons. In each case these strings are extended and a semi-classical approximation can be used. These mesons have masses of order the quark mass of their contents (i.e. not suppressed by the 't Hooft coupling). This separation in masses of different states is rather unlike QCD.

\section{Beyond the probe approximation (backreaction)}

The computations of quark and meson behaviour reviewed so far has been restricted to the probe brane approximation or, equivalently in the gauge theory, the quenched approximation. A significant limitation of the probe approximation is that the number of flavours must be much smaller than the number of colours, $N_{f} \ll N$. Similarly to the quenched approximation in (lattice) gauge theory, the probe approximation ignores the effects of the creation and annihilation of virtual quark-antiquark pairs on the gauge degrees of freedom. An obvious consequence of quenching is that potentially interesting quark contributions to the theory's $\beta$-function are lost. An unquenched computation, in which $N$ and $N_{f}$ are of the same order, requires that we go beyond the probe approximation. Virtual quark loops can be taken into account by including the backreaction of the flavour branes. In this section we discuss the simplest supersymmetric example of a supergravity solution which involves the backreaction of the flavour brane on the supergravity geometry. These computations are much harder than the probe computations and progress in more QCD-like theories is so far limited.

\subsection{Fully localized D3/D7 brane intersection}

As an example we consider the fully-localized D3/D7 intersection in flat space which has been constructed in a series of papers $[26,27,29,30]$. Before discussing the corresponding supergravity solution, we will review the D3/D7 worldvolume field theory at finite $N_{f} / N$, where, as in sect. $3, N$ and $N_{f}$ are the number of D3 and D7 branes, respectively.

\subsubsection{The $\mathcal{N}=2$ field theory at finite $N_{f} / N$}

Many aspects of the $\mathcal{N}=2$ field theory located on the D3/D7 brane intersection have already been discussed in sect. 3.1.1. The main difference from the quenched theory in the probe limit is that the theory has a positive oneloop beta function proportional to $\beta_{\mathcal{N}=2}^{\lambda} \sim \lambda^{2} \frac{N_{f}}{N}[30,75]$, i.e. it is not conformal. Since the theory is $\mathcal{N}=2$ supersymmetric, this is the exact (all-order) perturbative beta function - possible non-perturbative instanton contributions are ignored here. Note that the beta function vanishes in the conformal (probe) limit $\frac{N_{f}}{N} \rightarrow 0$ and the gauge coupling is constant, in agreement with the discussion in sect. 3. For finite values of the quotient $\frac{N_{f}}{N}$, the perturbative gauge coupling $\alpha=g_{Y M}^{2} / 4 \pi$ is given by

$$
\alpha\left(Q^{2}\right)=\frac{1}{\beta_{0} \log \frac{\Lambda_{L}^{2}}{Q^{2}}} \quad \text { with } \quad \Lambda_{L}^{2}=\mu^{2} e^{1 /\left(\alpha\left(\mu^{2}\right) \beta_{0}\right)}
$$

where $Q^{2}$ is the energy scale, $\mu^{2}$ a reference scale and $\beta_{0}=$ $N_{f} / 4 \pi$. The gauge coupling has logarithmic behaviour and runs into an ultraviolet Landau pole at the scale $\Lambda_{L}$.

Another interesting feature of the $\mathcal{N}=2$ theory, not present in the probe limit, is the chiral (or axial) anomaly. In the chiral limit $m_{q} \rightarrow 0$, the classical $\mathcal{N}=2$ theory features a chiral $U(1)_{\mathcal{R}}$ symmetry corresponding to $S O(2)$ rotations in $X^{8,9}$. At the quantum level, this symmetry is explicitly broken by the chiral anomaly which is proportional to

$$
\frac{N_{f}}{N} \operatorname{Tr} F \wedge F .
$$

The anomaly-free (unbroken) subgroup of $U(1)_{\mathcal{R}}$ is therefore the discrete group $\mathbb{Z}_{2 N f}$. This symmetry rotates the fundamental spinors as $\psi \rightarrow e^{-i \pi / N_{f}} \psi, \tilde{\psi} \rightarrow e^{-i \pi / N_{f}} \tilde{\psi}$, while the scalar $X=X^{8}+i X^{9}$ of the (adjoint) gauge multiplet transforms as $X \rightarrow e^{i 2 \pi / N_{f}} X$.

In sect. 4.1.2 we will discuss the dual supergravity description of the perturbative field theory ignoring instanton effects. The breaking of $U(1)_{\mathcal{R}}$ to $\mathbb{Z}_{2 N f}$ will not be visible in this solution. We will however come back to the chiral anomaly and its realization in the D3/D7 system in sect. 4.1.3. 


\subsubsection{The D3/D7 supergravity solution}

We will see that the running gauge coupling $\alpha\left(Q^{2}\right)$ and the non-trivial theta angle $\theta_{Y M}$ of the $\mathcal{N}=2$ theory can be recovered from the fully-localized D3/D7 intersection ${ }^{7}$. For simplicity, we will work in the case of massless quarks - the D7 branes are at the origin in the $\left(w_{5}, w_{6}\right)$-plane. The D3/D7 supergravity solution is given by

$$
\begin{aligned}
\mathrm{d} s_{10}^{2}= & h^{-1 / 2} \eta_{\mu \nu} \mathrm{d} x^{\mu} \mathrm{d} x^{\nu} \\
& +h^{1 / 2}\left(\mathrm{~d} \rho^{2}+\rho^{2} \mathrm{~d} \Omega_{3}^{2}+e^{-\phi}\left(\mathrm{d} w^{2}+w^{2} \mathrm{~d} \theta^{2}\right)\right),
\end{aligned}
$$

where the (near-core) warp factor $h=h(\rho, w)$ is ${ }^{8}$

$$
h(\rho, w)=1+\frac{R^{4}}{\left(\rho^{2}+e^{-\phi} w^{2}\right)^{2}},
$$

with $R^{4}=4 \pi g_{s} N \alpha^{\prime 2}$. The axion $\chi$ and dilaton $\phi$ are given by

$$
\chi(\theta)=\frac{N_{f}}{2 \pi} \theta, \quad e^{-\phi(w)}=\beta_{0} \log \frac{w_{\Lambda}^{2}}{w^{2}},
$$

where we choose the integration constant $w_{\Lambda}$ to be

$$
w_{\Lambda}^{2}=w_{0}^{2} e^{1 /\left(g_{s} \beta_{0}\right)}, \quad \beta_{0}=\frac{N_{f}}{4 \pi} .
$$

Here $x^{\mu}$ parameterizes the directions along the D3 branes (0123), while $\rho$ is the radial direction in the four-plane transverse to the D3 branes, but along the D7 branes (4567) and $w, \theta$ are the radial and angular direction in the two-plane transverse to the D7 branes. The background also contains the Ramond-Ramond four-form potential of the D3 brane solution. It has been shown in $[27,29]$ that this background preserves eight supercharges. For $N_{f}=0$, the background reduces to that of a stack of $N$ D3 branes (note here that $e^{\phi}=g_{s}$ and rescale $w^{2} \rightarrow w^{2} g_{s}$ ). For $N=0$, the background is that of $N_{f} \mathrm{D} 7$ branes.

Let us consider the case $N=0$ in more detail. Then, the warp factor becomes one, $h=1$, and we recover the D7 brane solution in the so-called weak-coupling approximation. Here the complete D7 brane dilaton profile [104] (shown as a dashed curve in fig. 13a) is approximated by the logarithmic profile (4.5) (solid curve in fig. 13a) at small radii $w$. Extrapolation of the logarithmic behaviour to larger values of $w$ shows an apparent dilaton divergence at the scale $w_{\Lambda}$.

This perfectly reflects the perturbative aspects of the dual $\mathcal{N}=2$ field theory, as can be seen as follows. Comparing (4.5) with (4.1), shows an intriguing similarity between the weak-coupling dilaton profile and the perturbative gauge coupling $[29,30]$. In fact, let us assume that

\footnotetext{
7 In a fully-localized brane solution the branes are located at a fix location in the transverse direction. One should compare this to a solution in which branes are "smeared" over some directions.

8 For simplicity, we restrict to the near-core region of the D3/D7 intersection (small values of $w$ ). The near-core approximation corresponds to the IR region of the dual field theory. The full warp factor is known in terms of a uniformly converging series expansion [30].
}
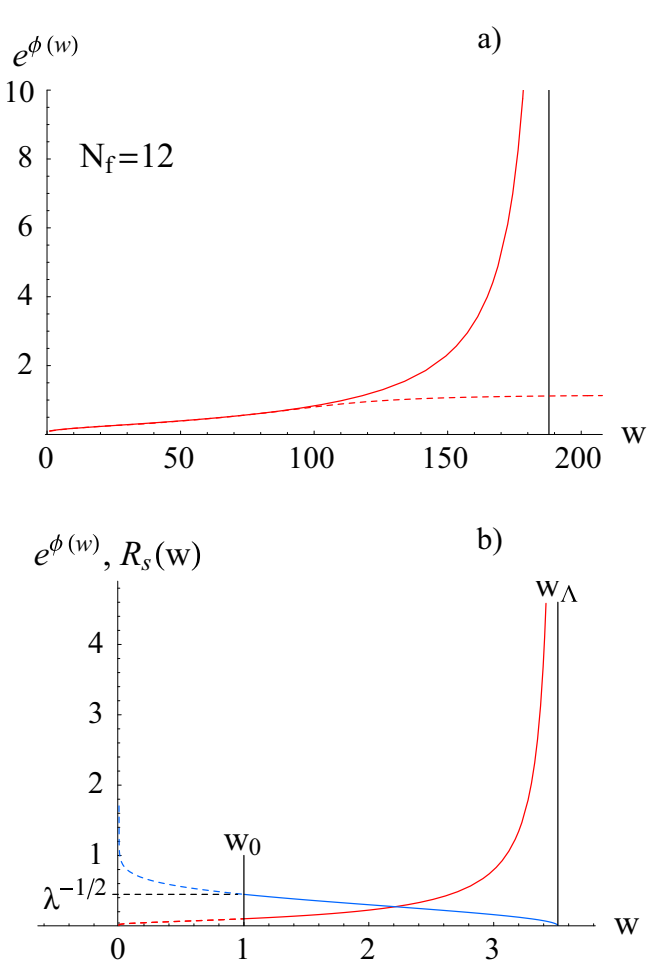

Fig. 13. a) The logarithmic (solid curve) and the full regular (dashed curve) dilaton profile (for $N_{f}=12, g_{s}=0.1, \theta=$ $\left.\pi / N_{f}\right)$. The regular profile approaches $e^{\phi}=2 / \sqrt{3}$ (i.e. $\tau=$ $j^{-1}(0)$ [104]) at $w \rightarrow \infty$. b) Dilaton (red curve) and curvature $\mathcal{R}_{s}(w)$ (blue curve) (for $N_{f}=N=50, g_{s}=0.1$ ).

the $w$-direction in the background corresponds to the energy scale $Q$ in the $\mathcal{N}=2$ theory, $Q=w /\left(2 \pi \alpha^{\prime}\right)$, and set $\mu=w_{0} /\left(2 \pi \alpha^{\prime}\right)$. If we then identify

$$
\begin{aligned}
\alpha\left(Q^{2}\right) & =g_{Y M}^{2}\left(Q^{2}\right) / 4 \pi=e^{\phi(w)}, \\
\alpha\left(\mu^{2}\right) & =g_{Y M}^{2}\left(\mu^{2}\right) / 4 \pi=g_{s},
\end{aligned}
$$

we observe that there is a direct correspondence between the running of the gauge coupling (4.1) and the logarithmic dilaton profile (4.5). Moreover, the string coupling $g_{s}=e^{\phi\left(w_{0}\right)}$ is fixed at $w_{0}$, not at infinity. This corresponds to fixing the gauge coupling $g_{Y M}^{2}\left(\mu^{2}\right)$ at some reference scale $\mu$. The above identification implies in particular that the UV Landau pole at $\Lambda_{L}\left(g_{Y M} \rightarrow \infty\right)$ is mapped to the dilaton divergence at $w_{\Lambda}, \Lambda_{L}=w_{\Lambda} /\left(2 \pi \alpha^{\prime 2}\right)$. Of course, here we map one pathology to another: The perturbative field theory becomes strongly coupled at the Landau pole $\Lambda_{L}$, while the supergravity solution breaks down at some distance $w_{\Lambda}$. In principle, both sides must be cured at these scales. This issue will be addressed in sect. 4.1.3.

We also find that the Yang-Mills theta angle $\theta_{Y M}$ is reflected by a non-trivial axion profile $\chi$ in the supergravity background, $\chi=\frac{\theta_{Y M}}{2 \pi}=\frac{\theta N_{f}}{2 \pi}$.

In order to obtain a supergravity theory dual to the $\mathcal{N}=2$ field theory, we consider the background (4.3) at large 't Hooft coupling $\lambda$ and fixed $N_{f} / N$. In this limit $(\lambda \gg 1)$, we may drop the " 1 " in the warp factor $h(\rho, w)$ and the D3 branes are replaced by their near-horizon ge- 
ometry. Note that the D7 branes do not disappear; open strings ending on the D7 branes are kept in this limit as signaled by the curvature singularity at the location $w=0$ of the $\mathrm{D} 7$ branes. (This singularity might be resolved within classical string theory by $\alpha^{\prime}$ corrections.)

The background is a good supergravity solution in the regime of small effective string coupling and small curvature, $e^{\phi} \ll 1$ and $\alpha^{\prime} \mathcal{R}_{s} \ll 1$. The first requirement is satisfied for radii $w \ll w_{\Lambda}$ corresponding to energies much below the Landau pole. The curvature measured in string units is $\alpha^{\prime} \mathcal{R}_{s} \sim \sqrt{e^{-\phi} / N}[27]$ and diverges for $w \rightarrow 0$. However, at the (infrared) cut-off $w_{0}(\propto \mu)$ the curvature becomes

$$
\alpha^{\prime} \mathcal{R}_{s} \sim \sqrt{\frac{1}{4 \pi} \frac{N_{f}}{N} \log \frac{w_{0}^{2}}{w^{2}}+\frac{1}{g_{s} N}} \stackrel{w=w_{0}}{=} \frac{1}{\sqrt{\lambda}},
$$

which is small in the large 't Hooft coupling limit. The background is thus a valid supergravity solution in the regime $w_{0} \leq w \ll w_{\Lambda}$ (corresponding to energies $\mu \leq$ $Q \ll \Lambda_{L}$ in the field theory). Figure 13 b shows a plot of the scalar curvature and the dilaton in this regime.

There are some subtleties to this construction which have been addressed at length in [30]. First, the dilaton diverges at $w_{\Lambda}$ and one might worry about the absence of a true boundary at $w \rightarrow \infty$. Note, however, that massless open string states (related to the field theory) precisely map into massless closed string states (generating supergravity) [30], i.e. there is no mixing with massive states. The gauge/gravity duality therefore works even without a true boundary. Second, since the D7 branes are codimension-two branes, there are uncancelled tadpoles in the string background. Tadpole divergences usually correspond to gauge anomalies and indicate an inconsistency in the theory. However, as it was found first in [105], logarithmic tadpoles do not correspond to gauge anomalies, but reflect the fact that the dual gauge theory is not conformally invariant. In fact, such tadpoles provide the correct one-loop running of the gauge coupling. Third, the full D7 brane geometry contains an asymptotic deficit angle of $2 \pi N_{f} / 12$ which restricts the number of flavours to $N_{f} \leq 12\left(N_{f}=24\right)[104]$. However, the background (4.3) is a valid supergravity solution for any $N_{f}$, which corresponds to the fact that there is no restriction on $N_{f}$ in the perturbative field theory. So, as long as we stay on the supergravity level and we do not want to extend (4.3) to a full string theory solution, we may use it for any $N_{f}$.

In summary, one finds that at large 't Hooft coupling $\lambda$ and fixed $N_{f} / N$ the D3/D7 solution (4.3) perfectly reflects the perturbative aspects of the dual $\mathcal{N}=2$ field theory at low energies, such as the ultra-violet Landau pole and the non-trivial theta angle.

\subsubsection{Non-perturbative completion and $U(1)_{\mathcal{R}}$ chiral anomaly}

The supergravity background discussed in the previous section describes only the perturbative regime of the $\mathcal{N}=2$ theory. In the strong-coupling regime the gauge coupling is



Fig. 14. Axion (blue dotted lines) and dilaton (red solid lines) for different values of $\theta\left(N_{f}=12\right)$.

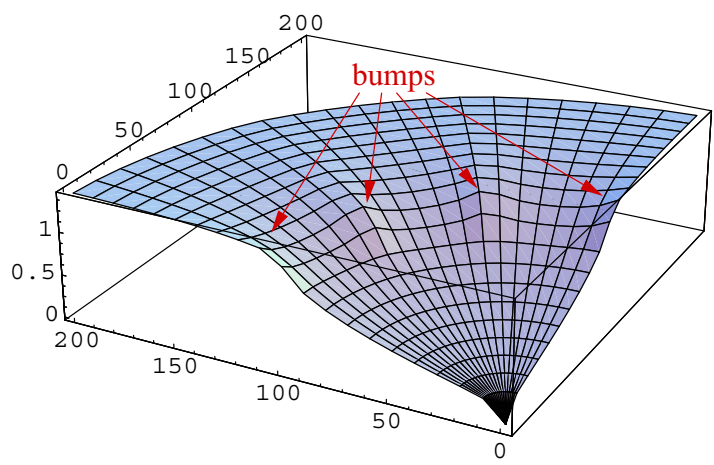

Fig. 15. "Bowl with bumps": D7 brane profile $\exp (\phi(w, \theta))$ (for $N_{f}=12$ flavours). Only the first quadrant is shown.

corrected by instanton contributions which may cure the ultra-violet Landau pole of the perturbative field theory. We now investigate to what extent a D3/D7 intersection including the full axion-dilaton of the D7 branes could represent an ultra-violet completion of the perturbative field theory, at least for $N_{f} \leq 12\left(N_{f}=24\right)$. We will refer to this intersection as the complete D3/D7 intersection as opposed to its logarithmic approximation discussed above.

The full axion-dilaton of the D7 branes is a solution to $j(\tau)=\left(w_{\Lambda} / w\right) e^{-i \theta}$, where $\tau=\chi+i e^{-\phi}$ and $j(\tau)$ is the modular $j$-function. Some solutions are plotted in fig. 14 which shows the profiles $e^{\phi(w)}$ and $\chi(w)$ for different values of the angular direction $\theta$. At small radii $w$ the dilaton has logarithmic behaviour and is independent of $\theta$. In the strong-coupling region at the scale $w_{\Lambda}$ the full D7 brane dilaton deviates from its logarithmic approximation. The profile also becomes dependent on $\theta$ leading to $N_{f}$ equally distributed "bumps" at the angles $2 \pi k / N_{f}$ ( $\left.k=1, \ldots, N_{f}\right)$, as shown in fig. 15. Asymptotically, the dilaton approaches the constant value $1 / \sin (2 \pi / 3) \approx 1.15$.

It is interesting to observe that the profile is invariant under rotations of $2 \pi / N_{f}$. This is a direct consequence of the chiral anomaly which breaks the chiral $U(1)_{\mathcal{R}}$ symmetry down to $\mathbb{Z}_{2 N_{f}}$. (Recall that the complex transverse direction parameterized by $X=w e^{i \theta}$ rotates under $\mathbb{Z}_{2 N_{f}}$ as $X \rightarrow e^{i 2 \pi / N_{f}} X$.) The chiral anomaly is a non-perturbative effect and can therefore not be seen in the logarithmic approximation. The full axion-dilaton profile of the D7 branes, however, nicely demonstrates this 
anomaly. This is an important consistency check of the complete D3/D7 intersection as a UV completion of the perturbative $\mathcal{N}=2$ theory. Note that so far we only discussed the axion-dilaton, but not the metric of the complete D3/D7 system. It is believed that in this case the warp factor $h(\rho, w)$ appearing in the metric ansatz (4.3) can only be computed numerically, which we will not do here.

\subsubsection{Meson computation}

A full computation of the mesonic spectrum of a backreacted solution is hard since one would need to look at fluctuations of the full geometry. Instead we will return to probe methods. We will embed a probe D7 brane into the backreacted geometry and study the scalar meson spectrum of the probe. This will at least provide some insight into the effects of flavour on the meson spectrum.

Firstly, we must check that it is possible to embed a probe D7 brane in the backreacted D3/D7 geometry (4.3). The D7 action takes the usual Dirac-Born-Infeld form (in Einstein frame), with a coupling to the field $C_{8}$ which is dual to the dilaton. We find

$$
S \sim \int \mathrm{d} \rho e^{\phi} \rho^{3}\left(\sqrt{1+e^{-\phi}\left(\partial_{\rho} w\right)^{2}}-1\right) .
$$

Note that the factors of $h$ cancel. The cancellation between the leading DBI term and the Wess-Zumino term is the usual cancellation that occurs for a D7 probe in the geometry of a stack of many D7 branes. This action is clearly minimized when $\partial_{\rho} w=0$ when the action is zero (one can also explicitly solve the equation of motion). The D7 probe therefore lies flat in the geometry just as in the usual probe computation - the value of $w$ the probe is placed at determines the quark mass.

If the quarks are all massless then the $\mathcal{N}=2$ gauge theory is conformal. We will therefore consider the case of $N_{f}$ backreacted (unquenched) quark flavours and a single quenched massive flavour. The scalar meson masses are given by fluctuations of the probe in the $w$-directions about its position $d$. The Lagrangian for such linearized fluctuations is given by

$$
\mathcal{L}=\frac{1}{2} \rho^{3} e^{\phi(d)}\left(\frac{R^{4}}{\left(\rho^{2}+e^{-\phi(d)} d^{2}\right)^{2}}\left(\partial_{x} \phi\right)^{2}+\left(\partial_{\rho} \phi\right)^{2}\right) .
$$

Up to an overall constant this is just the Lagrangian from the probe computation (3.12) but with

$$
d \rightarrow d\left(\beta_{0} \ln \frac{w_{\Lambda}^{2}}{d^{2}}\right)^{\frac{1}{2}}
$$

The meson spectrum is therefore, replacing $d=2 \pi \alpha^{\prime} m_{q}$ with $m_{q}$ the quark mass,

$$
M^{2}=\frac{8 \pi}{g_{s} N}\left(\beta_{0} \ln \frac{\Lambda^{2}}{m_{q}^{2}}\right) m_{q}^{2}(n+1)(n+2) .
$$

By eq. (4.7) the meson mass $M$ is proportional to

$$
\frac{g_{Y M}\left(\mu^{2}\right)}{g_{Y M}\left(m_{q}^{2}\right)}<1
$$

The effect of unquenching the quarks is just to replace the gauge coupling $g_{Y M}^{2}=4 \pi g_{s}$ with the appropriate renormalized value at the scale of the quark mass.

\section{More supersymmetric mesons}

There has been considerable work on including quark fields into gravity duals of gauge theories with less (but none zero) supersymmetry. Typically, these geometries are more complicated than AdS so even probe computations are hard work. We will be brief in our review of this work having spent considerable time on the simplest $\mathcal{N}=2$ theory and wishing to proceed to models with dynamical chiral symmetry breaking in the spirit of QCD. This section is intended as a guide to references for those who wish to pursue them.

\subsection{Klebanov's duals}

Klebanov, with a variety of collaborators, has studied models in which D3 branes and fractional D3 are placed on a conifold singularity [106-110]. A variety of $\mathcal{N}=1$ gauge theories with a product gauge group structure of the form $S U(N) \times S U(M)$ can be realized. The adjoint fields of the naive $S U(N+M)$ group on the D3 branes divide into adjoints of the sub-groups plus bi-fundamental fields. These theories display a chain of Seiberg dualities before developing a mass gap in the IR (corresponding to a deformation of the conifold).

D7 brane probes were first introduced into this theory in a supersymmetry preserving fashion adding extra massive quark supermultiplets in [111]. The meson spectrum was computed and displays a mass gap for the vector, scalar and pseudo-scalar mesons (see also $[112,113]$ ). A more complete set of probe embeddings were found in [114].

A perturbative analysis of the backreaction due to the introduction of D7 branes in [115] found evidence of Seiberg duality [116] in these theories.

Impressively, fully backreacted solutions for D7 branes in the $S U(N) \times S U(N)$ theory were constructed in [117]. Many of the symmetry properties of the theory were reproduced and the running gauge coupling correctly matches gauge theory expectations. These methods were extended to the general $S U(N) \times S U(M)$ theory in [118]. Again symmetry properties and the running gauge coupling of the field theory were correctly reproduced on the gravity side. Seiberg's duality is also manifest in the solutions. These techniques were also used to find embeddings in the geometry $A d S_{5} \times L^{a b c}$ in [119].

\subsection{B fields in the background: Polchinski-Strassler dual}

Interesting features arise if an antisymmetric two-form (which enters the supergravity theory as described in sect. 2.1) is turned on. The two-form enters the probe DBI 
action as in eq. (3.3). A prime example is the PolchinskiStrassler background [67], in which a $B$ field, together with a non-trivial $C_{2}$, is switched on in the six directions perpendicular to the boundary of $A d S_{5}$. The supersymmetry representation of $B$ is chosen such that the $B$ field is dual to mass terms for the adjoint chiral multiplets in the dual $\mathcal{N}=4$ gauge theory. In the supergravity picture, the D3 branes are polarized into D5 branes by virtue of the Myers effect [88]. The supergravity solution is only known as a perturbative expansion as one moves into the interior of the space, towards the IR of the field theory.

The embedding of D7 branes into this background for the so-called $\mathcal{N}=2^{*}$ and $\mathcal{N}=1^{*}$ theories, with a massive hypermultiplet and an equal mass for the three chiral multiplets, respectively, has been considered in [120] and [121]. This requires the explicit construction of the deformed gravity background to second order in the masses. For the $\mathcal{N}=2^{*}$ case, it was shown in [120] that the meson mass obtained from the D7 probe brane fluctuations receives a contribution from the adjoint scalar quark mass, such that there is a mass gap. Recently [122], the embedding of a D7 brane probe into the Lunin-Maldacena background [123] was considered, which is dual to a $\mathcal{N}=1$ supersymmetric marginal deformation of $\mathcal{N}=4$ super Yang-Mills theory. Here, a Zeeman-like spitting of the mass spectrum is observed.

\subsection{Maldacena-Nuñez dual}

The Maldacena-Nuñez background $[124,125]$ is dual to an $\mathcal{N}=1$ theory on the worldvolume of a D5 brane wrapped on a 2-sphere and therefore describes a relative of $\mathcal{N}=1$ Yang-Mills theory with additional Kaluza-Klein modes. The dual encodes the condensation of gauginos and the $N$ discrete vacua of the theory.

Probe D5 branes have been used to introduce matter fields into this theory with $\mathcal{N}=1$ supersymmetry preserved in $[126,127]$. The scalar and vector meson masses were computed numerically in [127] and are both compatible with the formula

$$
\begin{aligned}
& M_{n, l}=\sqrt{m^{2}\left(r_{*}, \lambda\right) n^{2}+l^{2}} \\
& m\left(r_{*}, \lambda\right)=\frac{\pi}{2 \Lambda}+r_{*}^{2}\left(\frac{0.23}{\Lambda}+\frac{0.53}{\Lambda^{3}}\right),
\end{aligned}
$$

where $n$ is the radial excitation number, $l$ the $\mathrm{R}$-charge, $r_{*}$ is a measure of the quark mass and $\Lambda$ is the strongcoupling scale of the underlying Yang-Mills theory.

A solution for the backreacted version of this theory has been found in [128]. The geometry encodes many of the properties of the theory including confinement and a running coupling.

Quarks and mesons are also investigated in an alternative $\mathcal{N}=2$ wrapped D5 brane theory in [129].

\subsection{Defect theories}

Probe techniques similar to those we have described for mesons have been used in gravity duals to include mat- ter fields in the fundamental representation in gauge theories on defects, i.e. on subspaces in $(2+1)$ or $(1+1)$ dimensions. The first such examples, in the $\mathcal{N}=4$ theory, were explored in [130-134]. Closed-form expressions for the masses of lower-dimension supersymmetric mesons in D3-D5 and D3-D3 systems were found in $[135,136]$. Other examples are in [137-140]. A backreacted D2-D6 system, in which both the adjoint and the fundamental degrees of freedom live in $(2+1)$ dimensions, can be found in [141], and further analysis is in [142].

\subsection{Non-commutativity}

Quarks have been introduced into non-commutative gauge theories using probe techniques in [143].

\section{Chiral symmetry breaking}

In the sections above we have introduced quarks into the basic AdS/CFT correspondence. These supersymmetric theories display bound mesonic spectra but, unlike in QCD, become conformal theories in the limit where the quark masses vanish. In QCD there is a dynamical mass generation mechanism (chiral symmetry breaking) that ensures the bound states remain massive as the quark masses fall to zero. In addition there are a special set of bound states, the pions, that are anomalously light because they are the (pseudo-)Goldstone bosons of the symmetry breaking. A gravity dual must capture these crucial pieces of dynamics if it is to describe QCD successfully - we describe a number of string constructions that achieve these goals in this section. In each case there is a very appealing geometric realization of the symmetry breaking providing a pleasing intuitive picture.

\subsection{Chiral symmetry breaking in field theory}

We begin with a brief summary of chiral symmetry breaking in gauge theory, in order to compare with the gravity description below. Consider the Lagrangian of massless QCD,

$$
\left.\mathcal{L}_{Q C D}\right|_{m=0}=-\frac{1}{4} F_{\mu \nu}^{a} F^{a \mu \nu}+\bar{\psi}_{L} \not D \psi_{L}+\bar{\psi}_{R} \not D \psi_{R},
$$

$\psi_{L}$ and $\psi_{R}$ are the chiral projections of the Dirac spinor $\psi$. In the massless case, the left-handed and right-handed fields have separate invariances under flavour symmetry. For the case of three flavours $u, d, s$ we have

$$
\psi_{L} \rightarrow \exp \left(-i \theta_{L} \cdot \lambda\right) \psi_{L}, \quad \psi_{R} \rightarrow \exp \left(-i \theta_{R} \cdot \lambda\right) \psi_{R},
$$

where $\lambda^{a}, a=1, \ldots, 8$ are the $S U(3)$ Gell-Mann matrices. These transformations may also be expressed as vector and axial-vector transformations,

$$
\psi \rightarrow \exp \left(-i \theta_{V} \cdot \lambda\right) \psi, \quad \psi \rightarrow \exp \left(-i \theta_{A} \cdot \lambda \gamma_{5}\right) \psi
$$


with $\theta_{V}=\left(\theta_{L}+\theta_{R}\right) / 2, \theta_{A}=\left(\theta_{L}-\theta_{R}\right) / 2$. The Lagrangian (6.1) is thus invariant under $S U(3)_{L} \times S U(3)_{R}$ or $S U(3)_{V} \times S U(3)_{A}$.

One might have expected a $U(3)_{L} \times U(3)_{R}$ global symmetry. It turns out that in QCD $U(1)_{A}$ is anomalous $[144,145]$, and thus not present in the quantum theory - gauge configurations with non-trivial winding number make $\partial_{\mu} J_{U(1)_{A}}^{\mu} \neq 0$ through the "triangle" quark loop graph. The only exception is when $N_{f} \ll N$ when the triangle graph becomes suppressed in a $1 / N$ expansion. The $U\left(N_{f}\right)_{A}$ symmetry is thus present at large $N$. The vector $U(1)$ is the baryon number symmetry and is a spectator to the symmetry breaking.

This chiral symmetry may be broken explicitly if a mass term is present in the Lagrangian,

$$
\mathcal{L}_{m}=-m \bar{\psi} \psi
$$

There is another spontaneous breaking of chiral symmetry in QCD though - the strong dynamics triggers the formation of a vev for the operator

$$
\langle\bar{\psi} \psi\rangle=\left\langle\bar{\psi}_{L} \psi_{R}\right\rangle+\text { h.c. } \neq 0 .
$$

In both symmetry-breaking cases, the flavour symmetry is broken down to a single vector $S U(3)_{V}$ factor,

$$
S U(3) \times S U(3) \rightarrow S U(3)_{V} .
$$

Goldstone's theorem though tells us that for a spontaneously broken symmetry 8 massless Goldstone bosons are expected, one for each broken generator. In QCD these are quark bound states, the $\pi^{ \pm}, \pi^{0}, K^{ \pm}, K^{0}, \bar{K}^{0}$ and the $\eta$. In the large- $N$ limit where the $U(1)_{A}$ symmetry is restored the $\eta^{\prime}$ joins these particles as a Goldstone boson.

A low-energy effective action for the Goldstone modes, which are lighter than all other QCD bound states, may be written (see for example [146]). The usual formulation is to write the Goldstone fields, $\pi^{a}$, as part of a field

$$
U=e^{i 2 \pi^{a} \lambda^{a} / f_{\pi}}
$$

where $f_{\pi}$ is the pion decay constant. $U$ transforms under the underlying chiral symmetries as $L^{\dagger} U R$ and its vev (the $3 \times 3$ unit matrix) breaks this symmetry to the diagonal. The effective Lagrangian can be constructed as a derivative expansion with leading term

$$
\mathcal{L}=f_{\pi}^{2} \operatorname{Tr} \partial^{\mu} U^{\dagger} \partial_{\mu} U+\ldots
$$

If a small explicit breaking by a quark mass term is present, the Goldstone bosons acquire mass to become pseudo-Goldstone bosons. Since the $3 \times 3$ mass matrix transforms under the (now spurious) chiral symmetries as $L^{\dagger} M_{q} R$ we can add a term to the low-energy action

$$
\Delta \mathcal{L}=\nu^{3} \operatorname{Tr} M_{q}^{\dagger} U
$$

where $\nu^{3}$ is some dimension-3 coefficient that measures the size of the quark condensate and must be fitted phenomenologically. This term generates a mass for the Goldstones with $M_{\pi}^{2} \sim M_{q}$.
We will see below how this symmetry breaking is realized in gravity duals. In the first examples, we will make use of the large- $N$ limit of the AdS/CFT correspondence and realize the breaking of a simple $U(1)_{A}$ symmetry, under which $\psi_{L}$ and $\psi_{R}$ transform as

$$
\psi_{L} \rightarrow e^{i \alpha} \psi_{L}, \quad \psi_{R} \rightarrow e^{-i \alpha} \psi_{R}
$$

The associated Goldstone boson has the quantum numbers of the $\eta^{\prime}$ particle although its behaviour is more akin to the pions. We will also describe a model that can realise the full non-Abelian chiral symmetry-breaking pattern as seen in QCD.

\subsection{D7 probes in non-supersymmetric backgrounds}

To see chiral symmetry breaking in the pattern of QCD in the AdS/CFT correspondence it is necessary to break supersymmetry completely. The operator $\bar{\psi} \psi$ is the F-term of a composite chiral superfield $Q Q$-its vev would break supersymmetry and so it would not be expected to be non-zero in a supersymmetric theory's ground state.

So far we have encountered two different generalizations of the AdS/CFT correspondence: On the one hand, the deformation of the $A d S_{5} \times S^{5}$ space, described in sect. 2.4, leads to holographic RG flows which, in particular cases, flow to confining gauge theories. On the other hand, we have discussed the addition of flavour arising from the addition of D7 brane probes in sect. 3 . The idea is now to combine these two generalizations of the AdS/CFT correspondence and to add D7 brane probes to deformed gravity backgrounds. As we discuss below, and first shown in [18], this leads to a dual gravity description of chiral symmetry breaking and Goldstone bosons.

\subsubsection{Constable-Myers background}

A prototype example for a confining gravity background in which supersymmetry is completely broken is the metric constructed by Constable and Myers in [52]. This background is an example of a dilaton flow (see also [69]), in which the dilaton - which is constant for the supergravity background dual to $\mathcal{N}=4$ super Yang-Mills - has a non-trivial profile, i.e. depends on the radial coordinate in deformed AdS space. At the supergravity level one simply searches for a solution of the IIB equations of motion with the dilaton switched on. More physical but also more complicated examples could be considered but this geometry provides an easy starting point. We will interpret the flow in terms of the field theory shortly ${ }^{9}$.

We choose a convenient coordinate system for the gravity background of Constable and Myers [52] such that in

\footnotetext{
9 Other very similar examples of chiral symmetry breaking by embedding $\mathrm{D} 7$ brane probes into different dilaton-flow geometries have been found, for instance, in $[91,147]$ and [148].
} 
Einstein frame, the geometry is given by

$$
\begin{aligned}
\mathrm{d} s^{2}= & H^{-1 / 2}\left(\frac{w^{4}+b^{4}}{w^{4}-b^{4}}\right)^{\delta / 4} \sum_{j=0}^{3} \mathrm{~d} x_{j}^{2} \\
& +H^{1 / 2}\left(\frac{w^{4}+b^{4}}{w^{4}-b^{4}}\right)^{(2-\delta) / 4} \frac{w^{4}-b^{4}}{w^{4}} \sum_{i=1}^{6} \mathrm{~d} w_{i}^{2}
\end{aligned}
$$

where $b$ is the scale of the geometry that determines the size of the deformation $\left(\delta=R^{4} /\left(2 b^{4}\right)\right.$ with $R$ the AdS radius) and

$$
H=\left(\frac{w^{4}+b^{4}}{w^{4}-b^{4}}\right)^{\delta}-1, \quad w^{2}=\sum_{i=1}^{6} w_{i}^{2} .
$$

In this coordinate system, the dilaton and four-form are, with $\Delta^{2}+\delta^{2}=10$,

$e^{2 \phi}=e^{2 \phi_{0}}\left(\frac{w^{4}+b^{4}}{w^{4}-b^{4}}\right)^{\Delta}, \quad C_{(4)}=-\frac{1}{4} H^{-1} \mathrm{~d} t \wedge \mathrm{d} x \wedge \mathrm{d} y \wedge \mathrm{d} z$

This geometry returns to $A d S_{5} \times S^{5}$ in the UV as may be seen by explicitly expanding at large $w$.

The field theory dual is therefore the $\mathcal{N}=4$ super Yang-Mills theory in the far UV. In the IR it is deformed by the parameter $b$ which sets the conformal symmetry breaking scale - it will determine the scale equivalent to $\Lambda_{Q C D}$ in the gauge theory

$$
\Lambda_{b}=\frac{b}{2 \pi \alpha^{\prime}} .
$$

The $S O(6)$ symmetry of the geometry is unbroken so the equivalent deformation in the gauge theory must not break the R-symmetry. We also see that $b$ enters with the radial direction in $\operatorname{AdS} w$ and $b^{4}$ must therefore correspond to an operator of dimension four. There is a natural dimension-four R-chargeless operator in the field theory which is $\operatorname{Tr} F^{2}$. This is a geometry therefore describing the $\mathcal{N}=4$ gauge theory with a source forcing it off its supersymmetric vacuum. Note that $\operatorname{Tr} F^{2}$ is the F-term of a composite operator of the product of two chiral superfields fields $W$ and hence a vev for the operator clearly breaks supersymmetry.

Note that the running of the dilaton in the gauge theory corresponds to a running coupling. Indeed the dilaton and geometry blow up at the scale $\Lambda_{b}$ consistent with the interpretation of that scale with $\Lambda_{Q C D}$. On the gravity side singularities ought to be identified with a source -in this non-supersymmetric case this identification is unclear but one might imagine that the D3 branes of the geometry have moved out from the origin to $b$ and complete the geometry. We will escape resolving this issue below because the D7 branes we will embed will not penetrate as far in as $b$.

This field theory of course has extra adjoint degrees of freedom as compared to QCD. However, it has been shown to be confining by calculating the Wilson loop, which has an area law. We will therefore take it as a model for a confining $S U(N)$ theory at large $N$.

The next step is to add quarks $[18,149]$. We will use an embedded probe D7 brane as discussed in sect. 3 . The D7 brane will be embedded, in the static gauge, with worldvolume coordinates identified with $x_{0,1,2,3}$ and $w_{1,2,3,4}$. Transverse fluctuations will be parameterized by $w_{5}$ and $w_{6}$-it is convenient to define a coordinate $\rho$ such that $\sum_{i=1}^{4} \mathrm{~d} w_{i}^{2}=\mathrm{d} \rho^{2}+\rho^{2} \mathrm{~d} \Omega_{3}^{2}$ and the radial coordinate is given by $w^{2}=\rho^{2}+w_{5}^{2}+w_{6}^{2}$.

In the field theory we have introduced $\mathcal{N}=2$ quark hypermultiplets with the usual superpotential coupling to the $\mathcal{N}=4$ fields which is $\tilde{Q} \Phi Q$. Note there is a $U(1)_{R}$ symmetry under which $Q$ and $\tilde{Q}$ have charge 0 (their fermionic components have charge -1 ) and $\Phi$ has charge +2 . This symmetry is analogous to $U(1)_{A}$ in that a vev for the fermionic quark bilinear $\bar{\psi} \psi$ will break the symmetry. Geometrically, this symmetry corresponds to rotations in the $\left(w_{5}, w_{6}\right)$-plane. With the supersymmetry breaking induced by $b^{4}$ in the geometry, we expect the scalar quarks in the hypermultiplets to become massive (that the moduli space is lifted and the scalar vevs pinned at zero was checked in [102]).

The Constable-Myers background is convenient for embedding a D7 brane probe since it preserves $S O(6)$ symmetry. The embedding functions determining the minimum energy configuration of the D7 probe are functions of $\rho$ only, i.e. essentially of the energy scale. As alluded to above the D7 brane probes giving rise to chiral symmetry breaking are embedded in a perfectly regular way avoiding the naked singularity in the IR at $b$.

The Dirac-Born-Infeld action of the D7 brane probe in the Constable-Myers background takes the form

$$
\begin{aligned}
& S_{\mathrm{D} 7}=-T_{7} R^{4} \int \mathrm{d}^{8} \xi \epsilon_{3} e^{\phi} \mathcal{G}\left(\rho, w_{5}, w_{6}\right) \\
& \times\left(1+g^{a b} g_{55} \partial_{a} w_{5} \partial_{b} w_{5}+g^{a b} g_{66} \partial_{a} w_{6} \partial_{b} w_{6}\right)^{1 / 2}
\end{aligned}
$$

where

$$
\begin{aligned}
& \mathcal{G}\left(\rho, w_{5}, w_{6}\right)= \\
& \rho^{3} \frac{\left(\left(\rho^{2}+w_{5}^{2}+w_{6}^{2}\right)^{2}+b^{4}\right)\left(\left(\rho^{2}+w_{5}^{2}+w_{6}^{2}\right)^{2}-b^{4}\right)}{\left(\rho^{2}+w_{5}^{2}+w_{6}^{2}\right)^{4}} .
\end{aligned}
$$

Here we have rescaled $w$ and $b$ in units of $R$ as in [150] so that factors of $R$ only occur as an overall factor on the embedding Lagrangian. Note that the factors of $\alpha^{\prime}$ cancel between $R^{4}$ and $T_{7}$ leaving the free energy proportional to $1 / g_{s}$-in the usual 't Hooft limit $\left(N \rightarrow \infty\right.$ with $g_{s} N$ fixed) the free energy grows as $N$ as one would expect.

From these equations we derive the corresponding equation of motion. We look for classical solutions of the form $w_{6}=w_{6}(\rho), w_{5}=0$. The equation of motion reads

$$
\begin{aligned}
\frac{\mathrm{d}}{\mathrm{d} \rho} & {\left[\frac{e^{\phi} \mathcal{G}\left(\rho, w_{6}\right)}{\sqrt{1+\left(\partial_{\rho} w_{6}\right)^{2}}}\left(\partial_{\rho} w_{6}\right)\right] } \\
& -\sqrt{1+\left(\partial_{\rho} w_{6}\right)^{2}} \frac{\mathrm{d}}{\mathrm{d} w_{6}}\left[e^{\phi} \mathcal{G}\left(\rho, w_{6}\right)\right]=0 .
\end{aligned}
$$




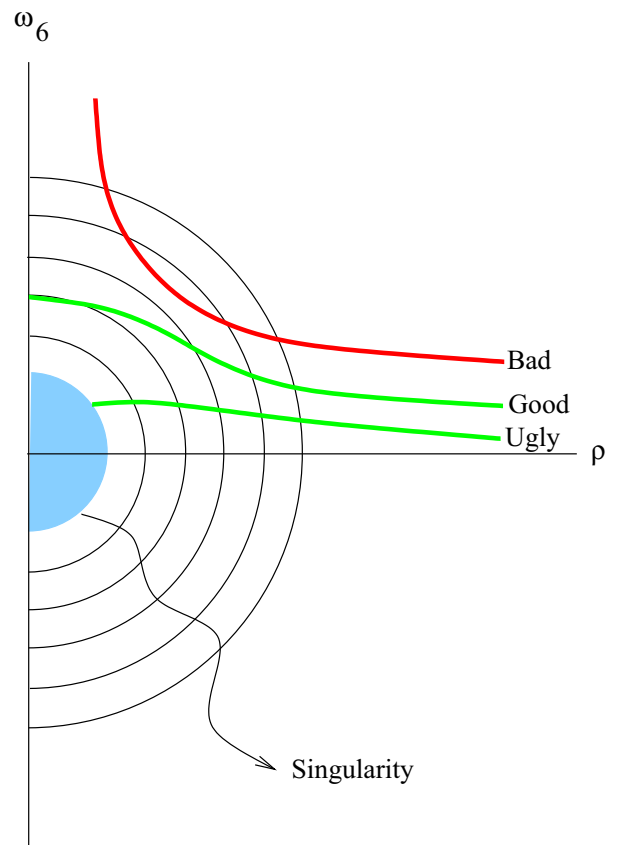

Fig. 16. Different possibilities for solutions of the D7 brane equations of motion. The semicircles are lines of constant $r$, which should be interpreted as a scale in the dual Yang-Mills theory. The "Bad" curve cannot be interpreted as an RG flow. The other curves have an RG flow interpretation, however the infrared (small $r$ ) region of the "Ugly" curve is outside the range of validity of supergravity. Figure from [18].

The last term in the above is a potential-like term that is evaluated to be

$$
\begin{aligned}
\frac{\mathrm{d}}{\mathrm{d} w_{6}}\left[e^{\phi} \mathcal{G}\left(\rho, w_{6}\right)\right]= & \frac{4 b^{4} \rho^{3} w_{6}}{\left(\rho^{2}+w_{6}^{2}\right)^{5}}\left(\frac{\left(\rho^{2}+w_{6}^{2}\right)^{2}+b^{4}}{\left(\rho^{2}+w_{6}^{2}\right)^{2}-b^{4}}\right)^{\Delta / 2} \\
& \times\left(2 b^{4}-\Delta\left(\rho^{2}+w_{6}^{2}\right)^{2}\right) .
\end{aligned}
$$

Numerically, we find solutions with the asymptotic behaviour $w_{6} \sim m+c / \rho^{2}$. The identification of these constants as field theory operators requires a coordinate transformation because the scalar kinetic term is not of the usual canonical AdS form. Transforming to coordinates $[16]$ in which the kinetic term has canonical form, we see that $m$ has dimension 1 and $c$ has dimension 3 . These coefficients are then identified with the quark mass $m_{q}$ and condensate $\langle\bar{\psi} \psi\rangle$, respectively, in agreement with the usual AdS/CFT dictionary obtained from the asymptotic boundary behaviour (2.15).

Due to the singularity in the background, we have to impose a regularity constraint on the brane embedding, which amounts to a boundary condition for the equation of motion determining the embedding. This is illustrated in fig. 16. Brane embeddings reaching the singularity are excluded since they enter a region of strong curvature where the supergravity approximation is no longer valid. In addition, embeddings which intersect the circles of constant energy twice cannot be interpreted as a RG flows and thus are unphysical. A boundary condition which selects the physical embeddings is to require the first derivative

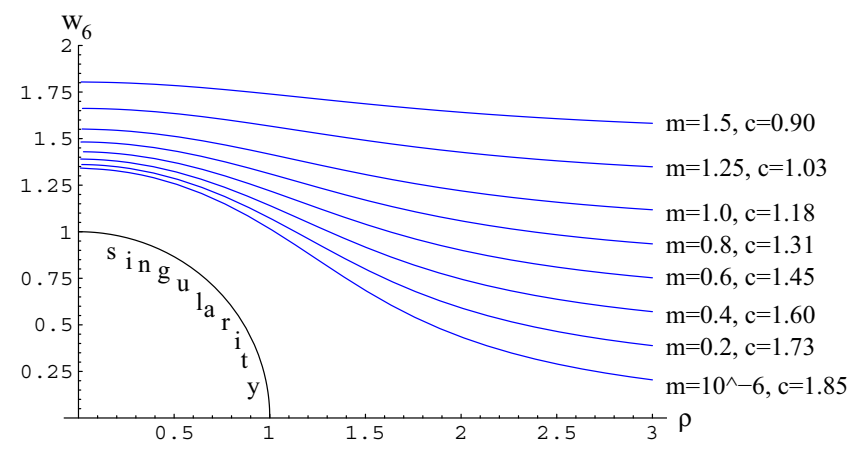

Fig. 17. Regular solutions in the Constable-Myers background. From [18].

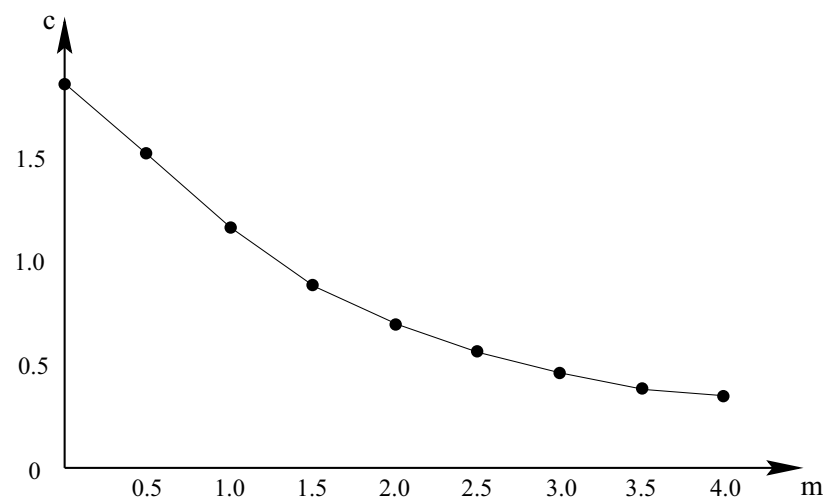

Fig. 18. A plot of the condensate parameter $c$ vs. quark mass $m$ for the regular solutions of the equation of motion in the Constable-Myers background. $c$ and $m$ are given in units set by the length scale $b$. From [18].

of the embedding functions to vanish at $\rho=0$. In the picture of the Karch/Katz RG flow discussed in sect. 3.2, this amounts to requiring the (now deformed) $S^{3}$ to shrink to zero at this point.

We now calculate the embedding functions for the D7 brane probe by solving the equations of motion obtained from the DBI action (6.15). The numerical result is displayed in fig. 17. For each of these embeddings we fix two boundary conditions, as required for solving a second-order differential equation: For regularity, we require the first derivative of the embedding to vanish at $\rho=0$. Secondly, the absolute value of the embedding function $w$ at the boundary $\rho \rightarrow \infty$ fixes the value of the quark mass in units of the scale $b$. The condensate $c \equiv\langle\bar{\psi} \psi\rangle$ in units of $b$ may then be read off from the asymptotic behaviour of the embedding at $\rho \rightarrow \infty$, where the embedding behaves as

$$
w \sim m+\frac{c}{\rho^{2}} .
$$

We see an interesting screening effect in fig. 17: The regular solutions appear to be repelled by the singularity, rather than just being straight lines as in the supersymmetric case. This can be related to spontaneous chiral symmetry breaking by a quark condensate: In fact, as is seen from figs. 17 and 18, there is a regular embedding with non-zero condensate even for $m \rightarrow 0$. This corre- 


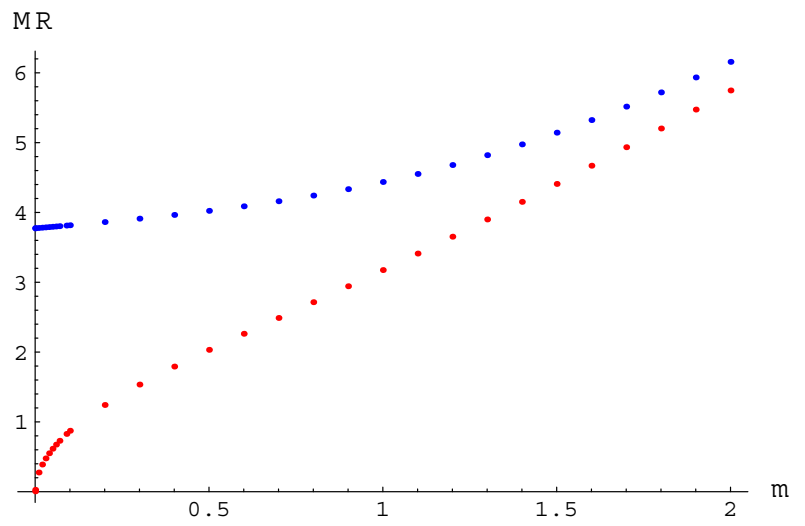

Fig. 19. Masses of the lowest-lying meson masses for fluctuations about the D7 brane embedding in radial and angular direction, as a function of the quark mass. The angular fluctuation mode gives rise to a (pseudo-)Goldstone mode. Since the spontaneously broken symmetry is $U(1)_{A}$, the Goldstone boson may be interpreted as the $\eta^{\prime}$, which is a Goldstone boson of this symmetry in $S U(N)$ gauge theory for $N \rightarrow \infty$. Figure provided by J. Große.

sponds exactly to spontaneous chiral symmetry breaking by a quark condensate! Moreover, at large $m$ we have $c \sim 1 / m$, as expected from field theory. Notice also the finite distance on the $w$-axis between the singularity and the embedding with $m \rightarrow 0$.

Remember that the D7 and D3 branes can in fact be separated in the full $\left(w_{5}, w_{6}\right)$-plane and therefore a D7 brane lying on the axis of that plane asymptotically (so the bare mass of the quark is zero and there is a good $U(1)_{A}$ symmetry in the UV) is deflected out onto any point on a circle in the plane. That circle represents the vacuum manifold of the breaking of the $U(1)$ symmetry -we see a completely geometric realization of the symmetry breaking.

\subsubsection{Goldstone boson}

Since there is spontaneous symmetry breaking for $m \rightarrow 0$, we expect a Goldstone boson analogous to the $\eta^{\prime}$, in the meson spectrum. Clearly, fluctuations in the angular direction in the $\left(w_{5}, w_{6}\right)$-plane (i.e. along the vacuum manifold) will generate these massless states. Solving the supergravity equation of motion for D7 probe brane fluctuations in the two directions transverse to probe, $\left(\delta w_{5}=f(r) \sin (k \cdot x), \delta w_{6}=h(r) \sin (k \cdot x)\right)$ around the D7 brane probe embedding shown in fig. 18, the meson masses are given by $M^{2}=-k^{2}$. There are indeed two distinct mesons (see fig. 19): One is massive for every $m$, and corresponds to fluctuations in the radial transverse direction, the other, corresponding to the $U(1)$ symmetric fluctuation, is massless for $m=0$ and is thus a Goldstone boson. It may be identified with the $\eta^{\prime}$, which becomes a $U(1)_{A}$ Goldstone boson for $N \rightarrow \infty$. At finite $N$, pure stringy corrections will give the $\eta^{\prime}$ a non-zero mass in the gravity picture, similarly to instantons in the field theory dual $[151,152]$.
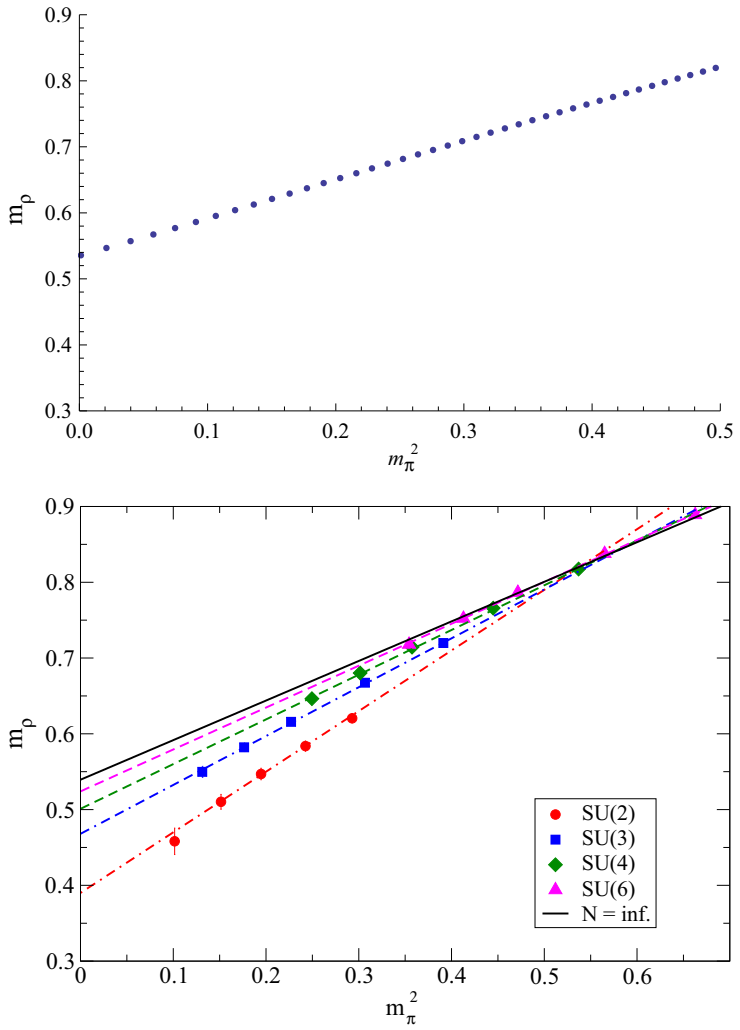

Fig. 20. A plot of $m_{\rho}$ vs. $m_{\pi}^{2}$ in the Constable-Myers background on top (we thank Andrew Tedder for generating this plot). Lattice data [20] (quenched and at finite spacing) for the same quantity is also shown at the bottom.

Another important property of the model of [18] is the small quark mass behaviour of the meson mass, proportional to the square root of $m$, thus satisfying the Gell-Mann-Oakes-Renner relation [153] of chiral QCD. Also the linear asymptotics for large $m$ correctly reproduce the field theory results. In [149] the scaling of the Goldstone's mass with the (small) quark mass was determined to be

$$
\frac{M_{\pi}^{2}}{\Lambda_{b}^{2}}=2.75 \sqrt{\frac{\pi}{g_{s} N}} \frac{m_{q}}{\Lambda_{b}} .
$$

\subsubsection{Vector mesons}

The vector mesons in the model are, as in the basic D7 brane embeddings, described by the gauge fields in the DBI action describing the D7 branes. Again solutions of the form $A^{\mu}=g(\rho) \sin (k \cdot x) \epsilon^{\mu}$ provide the masses of the $\rho$ and its radially excited states [149]. The $n=0$, unexcited, state has mass

$$
\frac{M_{\rho}^{2}}{\Lambda_{b}^{2}}=2.16 \sqrt{\frac{\pi}{g_{s} N}} .
$$

As expected it is massive, reflecting the dynamical generation of a quark mass.

In fig. 20 we plot the dependence of the rho meson mass on the pion mass squared in this model, in dimensionless 
units fixed by the choice of the supergravity scale $b$. The rho mass as a function of the pion mass squared has recently also been computed for large $N$ within lattice gauge theory $[19,20]$, and a direct comparison of gauge/gravity and lattice results is possible. In the lattice computations, the scale is set by the lattice spacing $a$. We choose our units such as to be able to compare directly with the lattice results of [20], which are shown at the bottom of fig. 20. In units such that the offset at $m_{\pi}=0$ coincides with [20], we find a linear dependence of the rho mass on the pion mass squared, with slope 0.57 .

For the lattice results of [20], the simulations are performed in the quenched approximation. This is appropriate for the large- $N$ limit, if not for smaller $N$. The lattice data of [20] is preliminary and at a fixed, finite lattice spacing. Nevertheless, it is striking that not only does the lattice data display the same linearity as the gauge/gravity model, but also the slope in the large- $N$ limit is 0.52 and therefore is very close to the gauge/gravity dual result. The fact that the numbers agree at the level of the first digit is surprising. We will see in the sections that follow that generically AdS-meson predictions match QCD data better than one would naively expect. For large values of the quark mass, we expect $m_{\rho} \propto m_{\pi}$ due to the onset of supersymmetry.

\subsection{Gauge theory in $\mathrm{AdS}_{4}$ space}

Another clean example of a gravity dual description of chiral symmetry breaking has been provided in [22]. There the duality is adapted to look at the $\mathcal{N}=4$ gauge theory in a four-dimensional anti-de-Sitter space. The gravity dual provided in [22] has a constant dilaton and is given by

$$
\begin{aligned}
\mathrm{d} s_{10}^{2}= & \left\{\frac{r^{2}}{R^{2}} A^{2}\left(-\mathrm{d} t^{2}+a(t)^{2} \gamma(x)^{2}\left(\mathrm{~d} x^{i}\right)^{2}\right)+\frac{R^{2}}{r^{2}} \mathrm{~d} r^{2}\right. \\
& \left.+R^{2} \mathrm{~d} \Omega_{5}^{2}\right\} \\
A=1+ & \left(\frac{r_{0}}{r}\right)^{2}, \quad a(t)=\frac{R^{2}}{2 r_{0}} \sin \left(2 \frac{r_{0}}{R^{2}} t\right) \\
\gamma(x)= & \frac{1}{1-x_{i} x^{i} /\left(4 \tilde{r}_{0}^{2}\right)},
\end{aligned}
$$

where $R=\sqrt{\Lambda} / 2=(4 \pi N)^{1 / 4}$ and $\tilde{r}_{0}$ is an arbitrary scale factor which sets the $4 \mathrm{~d}$ cosmological constant

$$
\lambda=-4 \frac{r_{0}^{2}}{R^{4}}
$$

The presence of a cosmological constant breaks both conformal invariance and supersymmetry.

Quarks are included in the geometry through probe D7 branes. The computation follows those already seen and we display the embeddings in fig. 21. Chiral symmetry breaking is clearly manifest.

The meson spectra associated with fluctuations of the D7 brane in the $w_{5}, w_{6}$-directions have also been computed and are shown in fig. 22. There is a pion mode, whose

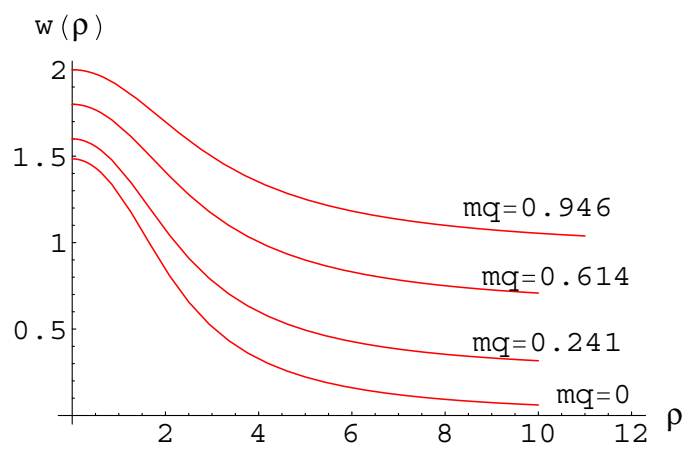

Fig. 21. D7 brane embeddings in the geometry with an $A d S_{4}$ subspace showing chiral symmetry breaking. Figure provided by K. Ghoroku.
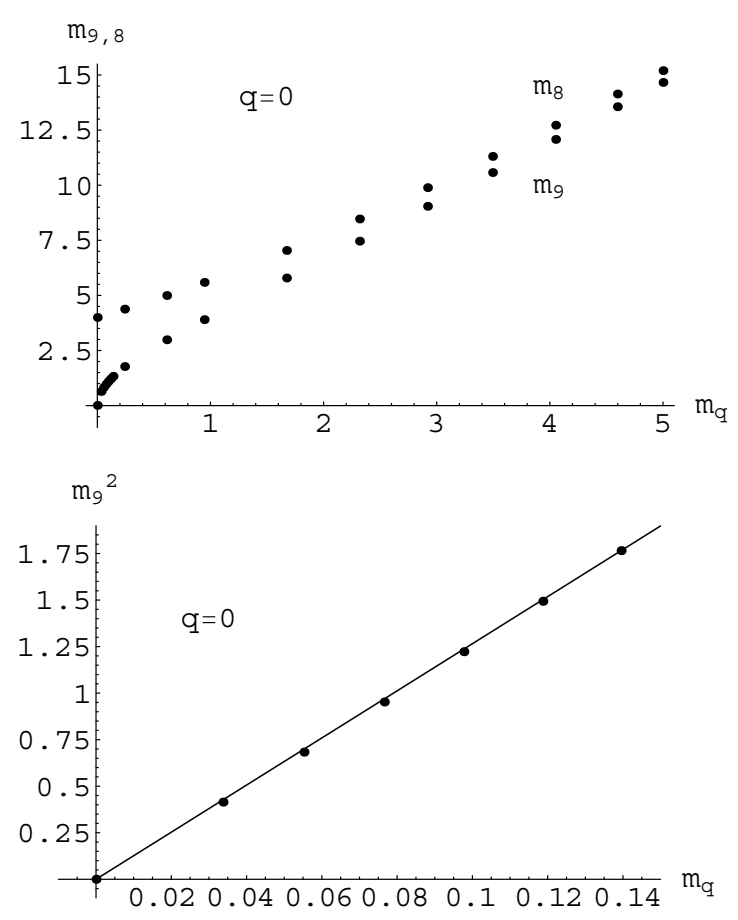

Fig. 22. Plot of the scalar $\left(m_{8}\right)$ and pseudo-scalar $\left(m_{9}\right)$ masses $v s$. the quark mass $m_{q}$ for $l=n=0, r_{0}=1.0$ and $R=1$ in the $A d S_{4}$ theory [22]. Plots provided by K. Ghoroku.

mass fits the expected Gell-Mann-Oakes-Renner relation for small quark mass, and a massive sigma mode.

D7 embeddings have also been studied when the gauge theory lives in de-Sitter space $[154,155]$ but there is no spontaneous chiral symmetry breaking in that case (the behaviour is like that of the $\mathcal{N}=4$ gauge theory at finite temperature described in sect. 7).

\subsection{Chiral symmetry breaking in the D4/D6 system}

A similar model based on a D4 brane background, in which one of the space directions wrapped by the D4 branes is compactified on a circle, was studied in [21]. There the flavour degrees of freedom are provided by D6 brane probes. Spontaneous chiral symmetry breaking of 
the $U(1)_{A}$ symmetry is seen in this model too. It has the advantage of not displaying a singularity in the interior of the curved space. On the other hand, the dual gauge theory becomes five-dimensional in the UV.

The authors of [21] consider the D4/D6 system with the branes oriented as described by the following array:

\begin{tabular}{|rc|c|c|c|c|c|c|c|c|c|c|}
\hline & 0 & 1 & 2 & 3 & 4 & 5 & 6 & 7 & 8 & 9 \\
\hline$N$ & $\mathrm{D} 4$ & $\mathrm{X}$ & $\mathrm{X}$ & $\mathrm{X}$ & $\mathrm{X}$ & $\mathrm{X}$ & & & & & \\
\hline$N_{f}$ & $\mathrm{D} 6$ & $\mathrm{X}$ & $\mathrm{X}$ & $\mathrm{X}$ & $\mathrm{X}$ & & $\mathrm{X}$ & $\mathrm{X}$ & $\mathrm{X}$ & & \\
\hline
\end{tabular}

The D4 and the D6 branes may be separated from each other along the 89-directions. On the gauge theory side one has a supersymmetric, five-dimensional $S U(N)$ gauge theory coupled to a four-dimensional defect. The entire system is invariant under eight supercharges, i.e. there is $\mathcal{N}=2$ supersymmetry in four-dimensional language. The degrees of freedom localized on the defect are $N_{f}$ hypermultiplets in the fundamental representation of $S U(N)$, which arise from the open strings connecting the D4 and the D6 branes. Each hypermultiplet consists of two Weyl fermions of opposite chiralities, $\psi_{L}$ and $\psi_{R}$, and two complex scalars.

Identifying the 4 -direction with period $2 \pi / M_{K K}$, and with anti-periodic boundary conditions for the D4 brane fermions, breaks all of the supersymmetries and renders the theory effectively four-dimensional at energies $E \ll$ $M_{K K}$. Further, the adjoint fermions and scalars become massive. Now, the bare mass of each hypermultiplet, $m_{q}$, is proportional to the distance between the corresponding D6 brane and the D4 branes. Even if these bare masses are zero, we expect loop effects to induce a mass for the scalars in the fundamental representation. Generation of a mass for the fundamental fermions is, however, forbidden by the chiral $U(1)_{A}$ symmetry. At low energies, one is therefore left with a four-dimensional $S U(N)$ gauge theory coupled to $N_{f}$ flavours of fundamental quark.

\subsubsection{D4 brane background}

The type-IIA supergravity background dual to $N$ D4 branes compactified on a circle with anti-periodic boundary conditions for the fermions takes the form

$$
\begin{aligned}
\mathrm{d} s^{2}= & \left(\frac{U}{R}\right)^{3 / 2}\left(\eta_{\mu \nu} \mathrm{d} x^{\mu} \mathrm{d} x^{\nu}+f(U) \mathrm{d} \tau^{2}\right) \\
& +\left(\frac{R}{U}\right)^{3 / 2} \frac{\mathrm{d} U^{2}}{f(U)}+R^{3 / 2} U^{1 / 2} \mathrm{~d} \Omega_{4}^{2}, \\
e^{\phi}= & g_{s}\left(\frac{U}{R}\right)^{3 / 4}, \quad F_{4}=\frac{N}{V_{4}} \varepsilon_{4}, \quad f(U)=1-\frac{U_{K K}^{3}}{U^{3}}
\end{aligned}
$$

The coordinates $x^{\mu}=\left\{x^{0}, \ldots, x^{3}\right\}$ parameterize the four non-compact directions along the D4 branes whereas $\tau$ parameterizes the circular 4-direction on which the branes are compactified. $\mathrm{d} \Omega_{4}^{2}$ and $\varepsilon_{4}$ are the $S O(5)$-invariant line element and volume form on a unit four-sphere, respectively, and $V_{4}=8 \pi^{2} / 3$ is its volume. $U$ has dimensions of length and may be thought of as a radial coordinate in the 56789-directions transverse to the D4 branes. To avoid a conical singularity at $U=U_{K K}, \tau$ must be identified with period

$$
\delta \tau=\frac{4 \pi}{3} \frac{R^{3 / 2}}{U_{K K}^{1 / 2}}
$$

This supergravity solution above is regular everywhere and is completely specified by the string coupling constant, $g_{s}$, the number of D4 branes $N$, and the constant $U_{K K}$. The remaining parameter, $R$, similar to the AdS radius, is given in terms of these quantities and the string length, $\ell_{s}$, by

$$
R^{3}=\pi g_{s} N \ell_{s}^{3} .
$$

The $S U(N)$ field theory dual to $(6.24),(6.25)$ is defined by the compactification scale, $M_{K K}$, below which the theory is effectively four-dimensional, and the four-dimensional coupling constant at the compactification scale, $g_{Y M}$. These are related to the string parameters by

$$
\begin{aligned}
M_{K K} & =\frac{3}{2} \frac{U_{K K}^{1 / 2}}{R^{3 / 2}}=\frac{3}{2 \sqrt{\pi}} \frac{U_{K K}^{1 / 2}}{\left(g_{s} N\right)^{1 / 2} \ell_{s}^{3 / 2}} \\
g_{Y M}^{2} & =3 \sqrt{\pi}\left(\frac{g_{s} U_{K K}}{N \ell_{s}}\right)^{1 / 2} .
\end{aligned}
$$

The string length cancels in any calculation of a physical quantity in the field theory. For example, the QCD string tension is

$$
\begin{aligned}
\sigma= & \left.\frac{1}{2 \pi \ell_{s}^{2}} \sqrt{-G_{t t} G_{x x}}\right|_{U=U_{K K}}= \\
& \frac{1}{2 \pi \ell_{s}^{2}}\left(\frac{U_{K K}}{R}\right)^{3 / 2}=\frac{2}{27 \pi} g_{Y M}^{2} N_{c} M_{K K}^{2} .
\end{aligned}
$$

\subsubsection{Probe D6 branes}

Flavour degrees of freedom are introduced into this model by adding D6 brane probes. Asymptotically (as $U \rightarrow \infty$ ), the D6 brane is embedded as described by (6.4). The analysis is simplified by introducing isotropic coordinates in the 56789-directions. A new radial coordinate $\rho$ is related to $U$ by

$$
U(\rho)=\left(\rho^{3 / 2}+\frac{U_{K K}^{3}}{4 \rho^{3 / 2}}\right)^{2 / 3}
$$

Moreover, five coordinates $\vec{z}=\left(z^{5}, \ldots, z^{9}\right)$ are introduced, such that $\rho=|\vec{z}|$ and $\mathrm{d} \vec{z} \cdot \mathrm{d} \vec{z}=\mathrm{d} \rho^{2}+\rho^{2} \mathrm{~d} \Omega_{4}^{2}$. In terms of these coordinates the metric $(6.24)$ becomes

$$
\mathrm{d} s^{2}=\left(\frac{U}{R}\right)^{3 / 2}\left(\eta_{\mu \nu} \mathrm{d} x^{\mu} \mathrm{d} x^{\nu}+f(U) \mathrm{d} \tau^{2}\right)+K(\rho) \mathrm{d} \vec{z} \cdot \mathrm{d} \vec{z}
$$

where

$$
K(\rho) \equiv \frac{R^{3 / 2} U^{1 / 2}}{\rho^{2}} .
$$

Here $U$ is now thought of as a function of $\rho$. Finally, to exploit the symmetries of the D6 brane embedding, 
it is useful to introduce spherical coordinates $\lambda, \Omega_{2}$ for the $z^{5,6,7}$-space and polar coordinates $r, \phi$ for the $z^{8,9}$-space. The final form of the D4 brane metric is then

$$
\begin{aligned}
\mathrm{d} s^{2}= & \left(\frac{U}{R}\right)^{3 / 2}\left(\eta_{\mu \nu} \mathrm{d} x^{\mu} \mathrm{d} x^{\nu}+f(U) \mathrm{d} \tau^{2}\right)+K(\rho) \\
& \times\left(\mathrm{d} \lambda^{2}+\lambda^{2} \mathrm{~d} \Omega_{2}^{2}+\mathrm{d} r^{2}+r^{2} \mathrm{~d} \phi^{2}\right),
\end{aligned}
$$

where $\rho^{2}=\lambda^{2}+r^{2}$. In these coordinates the D6 brane embedding takes a simple form, using $x^{\mu}, \lambda$ and $\Omega_{2}$ as worldvolume coordinates (or $\xi^{a}, a=1, \ldots, 6$ collectively). The D6 brane's position in the 89-plane is specified as $r=r(\lambda), \phi=\phi_{0}$, where $\phi_{0}$ is a constant. Note that $\lambda$ is the only variable on which $r$ is allowed to depend, by translational and rotational symmetry in the 0123- and 567-directions, respectively. Embeddings with $\tau=$ const correspond to a single D6 brane localized in the circle direction.

With this ansatz for the embedding, the induced metric on the D6 brane, $g_{a b}$, takes the form

$$
\begin{aligned}
\mathrm{d} s^{2}(g)= & \left(\frac{U}{R}\right)^{3 / 2} \eta_{\mu \nu} \mathrm{d} x^{\mu} \mathrm{d} x^{\nu}+K(\rho) \\
& \times\left[\left(1+\dot{r}^{2}\right) \mathrm{d} \lambda^{2}+\lambda^{2} \mathrm{~d} \Omega_{2}^{2}\right],
\end{aligned}
$$

where $\dot{r} \equiv \partial_{\lambda} r$. The D6 brane action becomes

$$
\begin{aligned}
& S_{\mathrm{D} 6}=-\frac{1}{(2 \pi)^{6} \ell_{s}^{7}} \int \mathrm{d}^{7} \xi e^{-\phi} \sqrt{-\operatorname{det} g}= \\
& -T_{\mathrm{D} 6} \int \mathrm{d}^{7} \xi \sqrt{h}\left(1+\frac{U_{K K}^{3}}{4 \rho^{3}}\right)^{2} \lambda^{2} \sqrt{1+\dot{r}^{2}}
\end{aligned}
$$

where $T_{\mathrm{D} 6}=2 \pi / g_{s}\left(2 \pi \ell_{s}\right)^{7}$ is the six-brane tension and $h$ is the determinant of the metric on the round unit twosphere. The equation of motion for $r(\lambda)$ is

$$
\begin{aligned}
\frac{\mathrm{d}}{\mathrm{d} \lambda}[ & {\left.\left[1+\frac{U_{K K}^{3}}{4 \rho^{3}}\right)^{2} \lambda^{2} \frac{\dot{r}}{\sqrt{1+\dot{r}^{2}}}\right]=} \\
& -\frac{3}{2} \frac{U_{K K}^{3}}{\rho^{5}}\left(1+\frac{U_{K K}^{3}}{4 \rho^{3}}\right) \lambda^{2} r \sqrt{1+\dot{r}^{2}} .
\end{aligned}
$$

Note that $r(\lambda)=r_{0}$, where $r_{0}$ is a constant, is a solution in the supersymmetric limit $\left(U_{K K}=0\right)$, as in $[16,17]$. This implies that there is no force on the D6 brane, regardless of its position in the 89-plane. The solution with $r_{0}=0$ preserves the $U(1)_{A}$ rotational symmetry in the 89-directions. If $U_{K K} \neq 0$, the force on the D6 brane no longer vanishes and causes it to bend, as dictated by the equation of motion above. In this case the $U(1)_{A}$ symmetry is broken. In particular, there is spontaneous symmetry breaking by a quark condensate, exactly in the same way as described in sect. 6.2.1 above for the D3/D7 system. Moreover, exactly as described in 6.2.2, there is a pseudo-Goldstone mode similar to the $\eta^{\prime}$. Mesons with large spin in this model are studied in the spirit of sect. 3.5 in $[93,156]$ and their decays are analyzed in [157]. Heavy-light mesons have been analyzed in [158].
For the case of multiple flavours, $N_{f}>1$, the authors of [21] present a holographic version of the Vafa-Witten theorem [159], which states that the $U\left(N_{f}\right)$ flavour symmetry cannot be spontaneously broken if $m_{q}>0$. In the holographic description this is realized by the fact that the $N_{f}$ D6 branes must be coincident in order to minimize their energy.

A novel feature of [21] is that there is a discussion of the case when both D6 and $\overline{\mathrm{D} 6}$ brane probes are present. This leads to a defect field theory in which the fundamental degrees of freedom are confined to a $(2+1)$-dimensional subspace. Nevertheless, this is an important step towards the physical model described in the next section.

\subsection{Non-Abelian chiral symmetries}

The holographic models we have reviewed to date are intrinsically supersymmetric gauge theories in the ultraviolet. This gives more control but means only that a $U(1)_{A}$ chiral symmetry can be realized because of couplings between the quarks and the adjoint, scalar superpartner of the gluons (there is a superpotential term $\tilde{Q} \Phi Q)$. To realize a more realistic non-Abelian chiral symmetry requires an analysis of an intrinsically nonsupersymmetric brane configuration - Sakai and Sugimoto $[24,25]$ have provided such a candidate.

The gauge degrees of freedom are provided, as in the model of sect. 6.4 , by a D4 brane with one direction wrapped on a circle. Quarks are included by including separated D 8 branes and anti-D8 branes. These D8 branes fill the whole space except one direction which is taken to be the circular direction. The model is displayed in fig. 23 - the compactified direction is $x^{4}$.

The D4-D8 ( $\overline{\mathrm{D} 8})$ strings generate chiral (anti-chiral) quark fields in the gauge theory [24]. The two $U\left(N_{f}\right)$ gauge symmetries on the surfaces of the $\mathrm{D} 8$ and $\overline{\mathrm{D}} 8$ branes are interpreted as the chiral non-Abelian flavour symmetries.

We should stress that the model describes a fivedimensional theory with chiral quarks living on defects in the UV. The compactified dimension renders a fourdimensional IR but we will not be able to drive the compactification scale smaller than the typical scale of the strong dynamics as one would like. Hopefully some universal features of this class of model can teach us about the four-dimensional gauge theory though.

We will see that the key feature of this model, when we take the strong coupling limit to render a gravity dual, is chiral symmetry breaking. In particular the D8 and $\overline{\mathrm{D} 8}$ branes will prefer to join into a single curved D8 brane as shown in fig. 23. There is only one surviving $S U(N)$ gauge symmetry corresponding to the chiral symmetries being broken to the vector. Furthermore there is a minimum D4-D8 separation so the quark strings stretched between them have some minimal dynamically determined mass.

\subsubsection{Gravitational background (D4-D8- $\overline{\mathrm{D} 8}$ )}

We can now consider the holographic dual of this D4-D8- $\overline{\mathrm{D} 8}$ system by taking the near horizon limit of the 

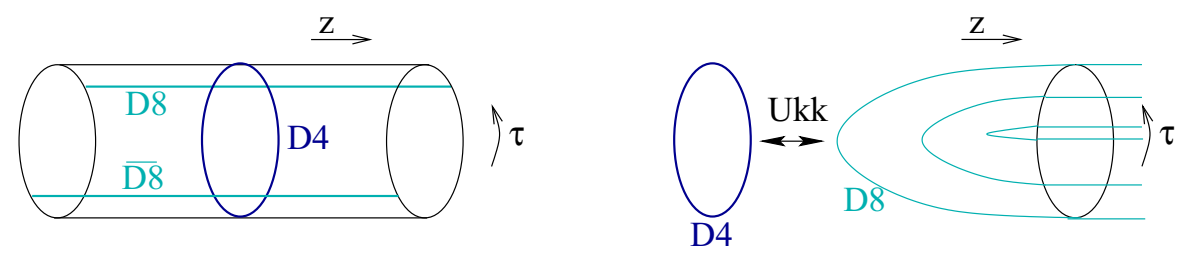

\begin{tabular}{|r|c|c|c|c|c|c|c|c|c|c|}
\hline & 0 & 1 & 2 & 3 & $(4)$ & 5 & 6 & 7 & 8 & 9 \\
\hline $\mathrm{D} 4$ & $\mathrm{X}$ & $\mathrm{X}$ & $\mathrm{X}$ & $\mathrm{X}$ & $\mathrm{X}$ & & & & & \\
\hline $\mathrm{D} 8-\overline{\mathrm{D}} 8$ & $\mathrm{X}$ & $\mathrm{X}$ & $\mathrm{X}$ & $\mathrm{X}$ & & $\mathrm{X}$ & $\mathrm{X}$ & $\mathrm{X}$ & $\mathrm{X}$ & $\mathrm{X}$ \\
\hline
\end{tabular}

Fig. 23. Sketch of the underlying D4-D8- $\overline{\mathrm{D} 8}$ brane construction and the chiral symmetry-breaking embeddings in the gravity dual.

geometry of a large- $N$ D 4 brane stack wrapped on a circle (note $\alpha^{\prime}$ corrections to the metric are considered in [160]). We have

$\mathrm{d} s^{2}=\left(\frac{u}{R}\right)^{\frac{3}{2}}\left(\mathrm{~d} x_{4}^{2}+f(u) \mathrm{d} \tau^{2}\right)+\left(\frac{R}{u}\right)^{\frac{3}{2}}\left(\frac{\mathrm{d} u^{2}}{f(u)}+u^{2} \mathrm{~d} \Omega_{4}^{2}\right)$

with $f(u) \equiv 1-\left(\frac{u_{K K}}{u}\right)^{3}$. Note here $u$ is the holographic direction. There is a non-zero four-form flux (not important for this discussion) and a dilaton $e^{-\phi}=g_{s}\left(\frac{u}{R}\right)^{-\frac{3}{4}}$.

Note the coordinate $\tau$ is periodic with the period given by $\delta \tau=\frac{4 \pi}{3} \frac{R^{\frac{3}{2}}}{u_{K K}^{\frac{1}{2}}}$ forming a $S^{1}$ which is wrapped by the D4 branes. This compactification is necessary in order to make the space-time smooth and complete. There is a horizon at $u=u_{K K}$ (where the radius of the $S^{1} \rightarrow 0$ ) which means the coordinate $u$ is restricted to the range $\left[u_{K K}, \infty\right]$. This scale represents the mass gap of the pure glue theory and the block to smaller $u$ shows the theory is confining.

We will change variables to the radial coordinate $z$, where $1+z^{2}=\left(\frac{u}{u_{K K}}\right)^{3}$, so the geometry becomes

$$
\begin{aligned}
\mathrm{d} s^{2}= & \left(\frac{u_{K K}}{R}\right)^{\frac{3}{2}}\left(\sqrt{1+z^{2}} \mathrm{~d} x_{4}^{2}+\frac{z^{2}}{\sqrt{1+z^{2}}} \mathrm{~d} \tau^{2}\right)+\left(\frac{R}{u_{K K}}\right)^{\frac{3}{2}} \\
& \times u_{K K}^{2}\left(\frac{4}{9}\left(1+z^{2}\right)^{-\frac{5}{6}} \mathrm{~d} z^{2}+\left(1+z^{2}\right)^{\frac{1}{6}} \mathrm{~d} \Omega_{4}^{2}\right) \cdot
\end{aligned}
$$

\subsubsection{Probe D8 branes}

As usual finding the full backreacted geometry when D8 branes are introduced is difficult so we will work in the probe limit corresponding to quenching in the gauge theory - a good approximation when $N_{f} \ll N$. The backreaction has been addressed as an expansion in the number of D8 branes in [161] and the probe embeddings below remain stable.

We can find the embeddings of a probe D8 brane in the above background. These form a family of curves in the $(z, \tau)$-plane which we parameterize as $\tau(z)$. The DiracBorn-Infeld (DBI) action for the embedding is

$$
S_{D B I}=\int_{\mathrm{D} 8} \mathrm{~d}^{8} \zeta e^{-\phi} \sqrt{-\operatorname{det}\left[\mathcal{P}\left(g_{a b}\right)\right]} .
$$

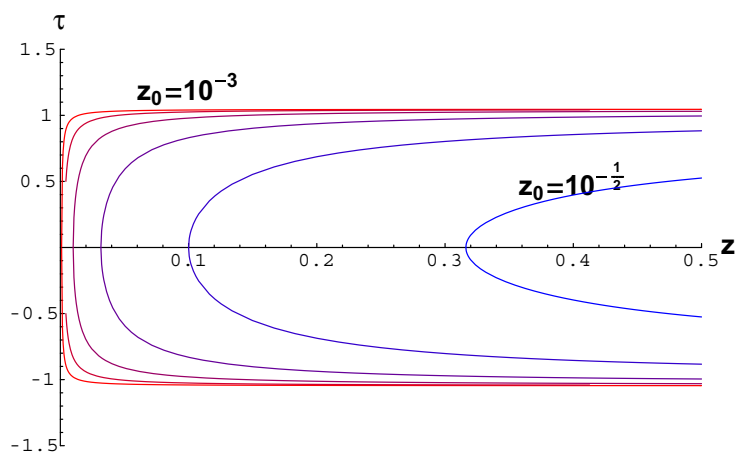

Fig. 24. Some regular D8 brane embeddings in the $(z, \tau)$ plane. We have set $R=1$ and $u_{K K}=1$ for the numerical plot.

This gives

$$
\begin{aligned}
& S_{D B I}=\operatorname{Vol}\left(S^{4}\right) \int \mathrm{d}^{4} x \int \mathrm{d} z \frac{2}{3} g_{s} u_{K K}^{5}\left(\frac{R}{u_{K K}}\right)^{\frac{3}{2}}\left(1+z^{2}\right)^{\frac{2}{3}} \\
& \times \sqrt{1+\frac{9}{4 u_{K K}^{2}}\left(\frac{u_{K K}}{R}\right)^{3} z^{2}\left(1+z^{2}\right)^{\frac{1}{3}} \tau^{\prime}(z)^{2}}
\end{aligned}
$$

One finds that the extremal configurations $\tau(z)$ for the D8 obey

$$
\begin{aligned}
\tau^{\prime}(z) & =\frac{2}{3}\left(\frac{R}{u_{K K}}\right)^{\frac{3}{2}} \\
\times & \frac{J}{\sqrt{u_{K K}^{6} g_{s}^{2} z^{4}\left(1+z^{2}\right)^{2}-J^{2} u_{K K}^{-2} z^{2}\left(1+z^{2}\right)^{\frac{1}{3}}}} .
\end{aligned}
$$

Here $J=g_{s} u_{K K}^{4} z_{0}\left(1+z_{0}^{2}\right)^{\frac{5}{6}}$ is chosen effectively to make the gradient infinite at $z=z_{0}$. This point is the point of closest approach of the $\mathrm{D} 8$ to the horizon at $u=u_{K K}$.

This gives us a one-parameter family of embeddings where choosing a particular value of $z_{0}$ specifies one particular curve. Some examples are shown in fig. 24 for $z_{0}$ increasing in factors of $\sqrt{10}$. Note the curve for $z_{0}=0$ consists of two horizontal pieces at $\tau= \pm \frac{\pi}{3} \frac{R^{\frac{3}{2}}}{u_{K K}^{\frac{1}{2}}}$ plus a vertical piece at $z=0$ connecting the two. The vertical piece lies on the horizon. 
The large $z$ (UV) asymptotic behaviour of the solutions takes the form

$$
\tau=\alpha-\frac{\beta}{z^{3}}
$$

with $\alpha, \beta$ free parameters.

\subsubsection{The pion}

For the moment let us restrict to discussing the $\beta=0$ solution (we will return to the other embeddings in sect. 6.5.5). For this solution the D8 and $\overline{\mathrm{D} 8}$ lie at anti-podal points on the circle until the connection along the horizon at $u=u_{K K}$. This configuration is interpreted as the theory with massless quarks and chiral symmetry breaking on the same scale as the mass gap of the glue. The chiral symmetry breaking should imply the existence of a Goldstone boson -in the one flavour case this will be the equivalent of the $\eta^{\prime}$ in QCD although since we are at large $N$ the anomaly is suppressed and the $\eta^{\prime}$ behaves more like a pion.

If chiral symmetry is broken there should be a vacuum manifold with different points corresponding to the different possible phases on the quark condensate. In [24] the phase of the quark condensate was identified with the value of the gauge field $A_{z}$ living on the D8 worldvolume. To identify the vacuum manifold we should find background solutions (that is, independent of the $x_{4}$ coordinates) for $A_{z}\left(z, x_{4}\right)$ which correspond to different global choices of the phase $\pi . A_{z}$ is described by the DBI action including a $U(1)$ gauge field, which at low energy has the Lagrangian density on the D8 worldvolume

$$
\mathcal{L}=e^{-\phi} \sqrt{-\operatorname{det}\left[\mathcal{P}\left(g_{a b}\right)\right]}\left(-1-\frac{1}{4} F^{a b} F_{a b}\right) .
$$

For the massless D8 brane embedding we can take $\tau(z)= \pm \frac{\delta \tau}{4}$ which evaluates to $\pm \frac{\pi}{3} \frac{R^{\frac{3}{2}}}{u_{K K}^{\frac{1}{2}}}$. Physically, the vertical part of the $\mathrm{D} 8$ brane in this case can be neglected because it lies along the horizon where points separated in $\tau$ are degenerate. Working on the upper branch of the D8 brane $\left(\tau(z)=+\frac{\pi}{3} \frac{R^{\frac{3}{2}}}{u_{K K}^{\frac{1}{2}}}\right)$ the action then takes the simple form (neglecting the volume factor coming from the foursphere angular coordinates —we are working with states of zero spin on the $S^{4}$ here)

$$
\begin{aligned}
S & =\frac{1}{2} \int_{0}^{\infty} \mathrm{d} z \int \mathrm{d}^{4} x\left(e^{-\phi} \sqrt{-g} g^{z z} g^{11}\right) \\
& \times\left(-\left(\partial_{0} A_{z}\right)^{2}+\left(\partial_{1} A_{z}\right)^{2}+\left(\partial_{2} A_{z}\right)^{2}+\left(\partial_{3} A_{z}\right)^{2}\right) .
\end{aligned}
$$

It is apparent that $F^{a b}$ and hence the action vanishes if $A_{z}$ is the only non-zero field and if it is only a function of $z$. Any function of $z$ is allowed. This is an artifact of gauge freedom in the model and one should pick a gauge. For example, one could gauge fix by including a term

$$
\delta \mathcal{L}=\frac{1}{\xi} e^{-\phi} \sqrt{-\operatorname{det}\left[\mathcal{P}\left(g_{a b}\right)\right]}\left(\nabla_{a} A^{a}-\kappa(z)\right)^{2},
$$

where $\kappa(z)$ is any arbitrary function. Writing $A_{z}\left(z, x_{4}\right) \equiv$ $g(z) \pi\left(x_{4}\right)$, there is sufficient freedom to pick any functional form of $g(z)$. We will follow the choice of Sakai and Sugimoto and pick

$$
g(z)=\frac{\mathcal{C}}{1+z^{2}} .
$$

The solution contains the arbitrary multiplicative factor $\mathcal{C}$ since the action is only quadratic in $A^{z}$. The freedom to pick the constant $\mathcal{C}$ in this solution is the freedom to move on the vacuum manifold.

We can now identify the pion field. It should correspond to space-time $\left(x^{\mu}\right)$ dependent fluctuations around the vacuum manifold. In other words we look at solutions of the form

$$
A_{z}(z, x)=\pi\left(x_{4}\right) \times \frac{2}{\sqrt{3 \pi}} \frac{1}{1+z^{2}} .
$$

Substituting this into the action (6.44) we find a canonically normalized kinetic term for a massless field,

$$
S=\int \mathrm{d}^{4} x \frac{1}{2}\left(\partial^{\mu} \pi\right)^{2}
$$

This is the pion - the Goldstone mode of the chiral symmetry breaking. The non-Abelian partners of this state are discussed in [162]. Note that interchanging the D 8 and $\overline{\mathrm{D} 8}$ branes corresponds to interchanging left- and right-handed quarks and is therefore a manifestation of parity in the model. This state has negative parity and is hence a pseudo-scalar.

\subsubsection{Meson spectrum and interactions}

Fluctuations of the D8 branes about the embeddings discussed correspond to mesons of the gauge theory. Generically, one looks for solutions of the linearized field equations coming from the DBI action of the form $f(u) e^{i k x}$. Even and odd functions $f(r)$ describe even- and odd-parity states.

Fluctuations of the vector field in the DBI action generate vector and axial vector mesons (note that the links to the ideas of a hidden local symmetry in QCD are made in [163]). In addition there is a scalar field corresponding to fluctuations of the embedding. If we restrict these fluctuations to the trivial harmonic of the four-sphere on the D8 transverse to the $x$-directions, we obtain QCD-like states. It is important to realize there are additional states with higher harmonics that effectively have R-charge indicating that there are light non-degenerate "superpartners" of the QCD fields in the field theory. There are, in addition, fermionic fields in the DBI action that would describe mesinos if supersymmetry were restored [164]. Finally, there are also Kaluza-Klein modes of the glueballs and gluino balls from the gauge sector. The typical scale for the masses of all of these bound states is

$$
M_{K K}=\frac{3}{2} \frac{U_{K K}^{1 / 2}}{R^{3 / 2}}, \quad R^{3 / 2}=\sqrt{\pi g_{s} N l_{s}^{3}} .
$$


Note that as in previous examples, the mesons are tightly bound in the limit $g_{s} N \rightarrow \infty$, and hence rather un-QCD-like. The values of the masses for states that can be mapped to QCD have been computed in [24,25]. They find

$$
\begin{array}{cccc}
m_{\rho} & 0.67 M_{K K}, & m_{a_{1}} & 1.58 M_{K K}, \\
m_{\rho}^{*} & 1.89 M_{K K}, & m_{a_{1}^{*}} & 2.11 M_{K K}, \\
m_{\rho}^{* *} & 2.21 M_{K K} . & &
\end{array}
$$

The interactions between mesons can also be computed by inserting the functions $f(r)$ back into the DBI action and integrating over the four non-spatial directions on the D8. Some example values are

$$
\begin{aligned}
& f_{\pi}^{2}=\frac{1}{54 \pi^{4}} g_{Y M}^{2} N^{2} M_{K K}^{2}, \\
& g_{\rho \rho \rho}=0.45 \frac{(6 \pi)^{3 / 2}}{g_{Y M} N}, \\
& g_{\rho \pi \pi}=0.42 \sqrt{\frac{216 \pi^{3}}{g_{Y M}^{2} N^{2}}} .
\end{aligned}
$$

If one forces this model onto the QCD spectrum by fitting the scale $M_{K K}$ and $g_{Y M}^{2} N$, then these results match the data at the $20-30 \%$ level.

Purely pionic interaction terms exist as well which reproduce a Skyrme style model of baryons. There has been considerable interest in the baryonic sector of the model recently - see the papers [165-172]. See also the work on introducing a Fermi surface at high density $[173,174]$.

\subsubsection{Non-anti-podal embeddings}

Finally, we return to the non-anti-podal embeddings of fig. 24. Clearly, these embeddings have a larger D4-D8 separation and hence a larger quark mass - there remains debate in the literature about whether this mass is a hard mass [175] or entirely dynamically generated $[138,176,177]$.

The configurations differ in their asymptotic positions of the D8 branes. Perturbatively, the configuration of a D8 and $\overline{\mathrm{D} 8}$ on a circle is not generically stable due to their attraction and they would be expected to join, suggesting chiral symmetry breaking is present at weak coupling. Fluctuations of the D8s in the UV though correspond to strings with both ends on one D8 and are hence dual to operators in the adjoint of the $U\left(N_{f}\right)$ chiral flavour symmetries. For example, they could correspond to the coupling and source of a (possibly higher dimension) operator of the form $\bar{q}_{L} \gamma^{\mu} D_{\mu} q_{L}$. Clearly dialing this coupling in the UV Lagrangian would enhance the gluon exchange diagram between quarks and might well increase the dynamically generated mass. On the other hand, if the true parameter that is being changed is the quark mass then dynamically that could feed through to set a different value for the same operator's coupling and vev. The change in position of the D8 may be an indirect signal of the presence of a quark mass.
One would think that the difference between hard and explicit chiral symmetry breaking should be evident from the existence, or otherwise, of a flat direction in the potential. For all of these configurations the gauge freedom discussed in sect. 6.5.3 above remains, so the analysis there shows there is a flat direction. However, in the full string construction the quark mass is a field vev and there should be a larger spurious symmetry of the form

$$
q_{l} \rightarrow e^{i \alpha} q_{L}, \quad q_{R} \rightarrow e^{-i \alpha} q_{R}, \quad m \rightarrow e^{-2 i \alpha} m .
$$

If the flat direction corresponds to this symmetry then fluctuations in this direction are not physical modes in the gauge theory in which the phase of the mass is fixed.

Another approach taken has been to include an explicit tachyonic mode connecting the D8 and $\overline{\mathrm{D} 8}$ in [178-180]. This field should directly describe the quark mass and condensate and its vacuum solution does indeed set the shape of the linked $\mathrm{D} 8-\overline{\mathrm{D} 8}$ pair. The precise tachyon potential is not known though.

In practice for the spectrum (the pion apart) it is not too important whether the mass is dynamical or hard. All the meson masses rise as the D8s are brought closer together asymptotically.

An alternative attempt to introduce a quark mass by the introduction of an instanton on the D8 worldvolume can be found in [181].

\subsection{More chiral symmetry breaking}

A number of other examples of holographic chiral symmetry breaking exist in the literature. In [150] probe D7 branes were numerically embedded in the nonsupersymmetric Yang-Mills* deformation [71] of the $\mathcal{N}=$ 4 theory providing evidence for chiral symmetry breaking. Quarks have also been added to the beta-deformed $\mathcal{N}=4$ theory in [122] - chiral symmetry breaking is again observed. It appears to be generically true that breaking supersymmetry in gravity duals leads to chiral symmetry breaking.

Attempts have been made to construct gravitational duals of QCD in non-critical string theories. These gravity theories in less than ten dimensions risk the presence of order-one curvature and so are not completely controlled. Nevertheless, the $A d S_{6}$ Schwarzschild black hole [182] is a possible candidate and shows confining behaviour. In [183] quarks are introduced in the spirit of sect. 6.5 above via both a D4- $\overline{\mathrm{D} 4}$ and D5- $\overline{\mathrm{D} 5}$. Chiral symmetry breaking is observed in the pattern of the Sakai-Sugimoto model and the vector meson masses have been computed. A non-critical D3-D4- $\overline{\mathrm{D} 4}$ configuration is discussed in [184] and again displays chiral symmetry breaking.

\subsection{Summary}

We have reviewed a number of holographic descriptions of chiral symmetry breaking. Quarks are introduced via probe branes in non-supersymmetric geometries. In each 
case, the repulsion of the core geometry acting on the probes causes the spontaneous symmetry breaking, which appears as a manifest breaking of a symmetry in the geometrical set-up. The quarks acquire a dynamical mass resulting in a non-zero vector meson mass even at zero quark mass. Goldstone bosons of the symmetry breaking play the role of the pions of QCD. These are all crucial dynamical results in view of holographically describing QCD.

\section{Mesons at finite temperature}

In previous sections we have focused on strongly coupled gauge theories at zero temperature. Considerable progress has also been made on understanding the thermal properties of gauge theories using holography.

The gravitational dual of placing the $\mathcal{N}=4$ gauge theory at finite temperature is to replace the AdS space with an AdS-Schwarzschild black hole [31,32]. The black hole has all the correct thermodynamic properties to describe the thermodynamics of the gauge theory. Further, the horizon cuts off the holographic radial direction corresponding to cutting off energy scales below that of the temperature in the field theory. In the infinite volume limit, the free energy of the black-hole solutions is lower than that of AdS with a compact time direction for any temperature. If the spatial directions of the theory are also compact, the transition between AdS space and the AdSSchwarzschild black hole can be shifted to higher temperatures of order the inverse compactification scale. This gravitational tunnelling transition was first described by Hawking and Page [185]. Witten has interpreted it, within the gauge-gravity duality, as the dual of the deconfinement transition. The free energy of AdS scales as order one relative to the black-hole geometry's free energy which scales as $N^{2}$ - the high-temperature phase has deconfined gluons (and superpartners).

A considerable amount of work has been done on the holographic description of the transport properties of the quark-gluon plasma. Amongst these is the famous ratio of shear viscosity to volume density of entropy which takes the value $\hbar / 4 \pi k_{B}$ [186]. This corresponds to the "fluid" with the lowest-known value of this ratio. There has been considerable interest in this quantity, since the value of this ratio deduced from RHIC heavy-ion collisions suggests that the quark-gluon plasma is an almost perfect fluid of this type [187].

Here we will constrain ourselves to reviewing results on the thermal properties of mesons using the AdS/CFT correspondence $[18,34-36]$.

\subsection{First-order phase transition in the quark-gluon plasma}

An interesting new first-order phase transition has been found which occurs as the temperature increases and passes through the meson's mass scale. At this scale the meson disassociates, or melts, into the background plasma. This is an additional transition at energy scales above the deconfinement scale. The sharp transition is probably a consequence of being at large $N$ and is not believed to be present in QCD on the basis of lattice results [188-190]. Nevertheless, the gauge/gravity dual description does allow the study of meson melting.

\subsubsection{AdS-Schwarzschild solution}

The high temperature, deconfined, phase of the $\mathcal{N}=4$ gauge theory is described by the AdS-Schwarzschild solution, given by

$$
\mathrm{d} s^{2}=\frac{K(r)}{R^{2}} \mathrm{~d} \tau^{2}+R^{2} \frac{\mathrm{d} r^{2}}{K(r)}+\frac{r^{2}}{R^{2}} \mathrm{~d} \vec{x}^{2}+R^{2} \mathrm{~d} \Omega_{5}^{2},
$$

where

$$
K(r)=r^{2}-\frac{r_{H}^{4}}{r^{2}} .
$$

Asymptotically for $r \gg r_{H}$, the black-hole solution approaches $A d S_{5} \times S^{5}$ whose radius is related to the 't Hooft coupling of the dual gauge theory by $R^{4}=4 \pi \lambda \alpha^{\prime 2}$. This space-time is smooth and complete if $\tau$ is periodic with period $\pi r_{H}$. Note that the $S^{1}$ parameterized by $\tau$ collapses at the horizon $r=r_{H}$. The fact that the geometry "ends" at $r=r_{H}$ is responsible for the existence of an area law for the Wilson loop and a mass gap in the dual field theory (see [32]). For convenience, in the numerical work below we shall set both $R$ and $r_{H}$ equal to 1 .

The temperature of the field theory corresponds to the Hawking temperature of the black hole which is given by the radius of the horizon, $T=r_{H} /\left(R^{2} \pi\right)$. At finite temperature the fermions have anti-periodic boundary conditions in the Euclidean time direction [32] and supersymmetry is broken. The black hole solution thus describes a strongly interacting quark-gluon plasma which is non-supersymmetric and non-conformal. It is therefore believed that, despite the presence of other fields not contained in QCD, this plasma shares some properties with the quark-gluon plasma of QCD.

As in the previous sections, we now introduce a D7 brane into this background, which corresponds to the addition of matter in the fundamental representation. The dual field theory is the $\mathcal{N}=2$ gauge theory discussed in sect. 3.1.1, but now at finite temperature.

\subsubsection{Embedding of a D7 brane}

To embed a D7 brane in the AdS black-hole background it is useful to recast the metric (7.1) to a form with an explicit flat 6 -plane. To this end, we change variables from $r$ to $w$, such that

$$
\frac{\mathrm{d} w}{w} \equiv \frac{r \mathrm{~d} r}{\left(r^{4}-r_{H}^{4}\right)^{1 / 2}},
$$

which is solved by

$$
2 w^{2}=r^{2}+\sqrt{r^{4}-r_{H}^{4}} .
$$


The metric is then

$$
\begin{aligned}
\mathrm{d} s^{2}= & \left(w^{2}+\frac{w_{H}^{4}}{w^{2}}\right) \mathrm{d} \vec{x}^{2}+\frac{\left(w^{4}-w_{H}^{4}\right)^{2}}{w^{2}\left(w^{4}+w_{H}^{4}\right)} \mathrm{d} t^{2} \\
& +\frac{1}{w^{2}}\left(\sum_{i=1}^{6} \mathrm{~d} w_{i}^{2}\right)
\end{aligned}
$$

where $\sqrt{2} w_{H}=r_{H}, \sum_{i} \mathrm{~d} w_{i}^{2}=\mathrm{d} w^{2}+w^{2} \mathrm{~d} \Omega_{5}^{2}$, which, for reasons of convenience, will also be written as $\mathrm{d} \rho^{2}+$ $\rho^{2} \mathrm{~d} \Omega_{3}^{2}+\mathrm{d} w_{5}^{2}+\mathrm{d} w_{6}^{2}$, where $\mathrm{d} \Omega_{3}^{2}$ is the unit three-sphere metric. The AdS black-hole geometry asymptotically approaches $A d S_{5} \times S^{5}$ at large $w$. Here the background becomes supersymmetric, and the D7 embedding should approach the constant solutions $w_{6}=m=$ const, $w_{5}=0$ found in sect. 3. To take into account the deformation, we will consider a more general ansatz for the embedding of the form $w_{6}=w_{6}(\rho), w_{5}=0$, with the function $w_{6}(\rho)$ to be determined numerically. The DBI action for the orthogonal directions $w_{5}, w_{6}$ is

$$
\begin{aligned}
S_{\mathrm{D} 7}= & -\mu_{7} \int \mathrm{d}^{8} \xi \epsilon_{3} \mathcal{G}\left(\rho, w_{5}, w_{6}\right) \\
& \times\left(1+\frac{g^{a b}}{\left(\rho^{2}+w_{5}^{2}+w_{6}^{2}\right)} \partial_{a} w_{5} \partial_{b} w_{5}\right. \\
& \left.\quad+\frac{g^{a b}}{\left(\rho^{2}+w_{5}^{2}+w_{6}^{2}\right)} \partial_{a} w_{6} \partial_{b} w_{6}\right)^{1 / 2},
\end{aligned}
$$

where the determinant of the metric is given by

$$
\begin{aligned}
& \mathcal{G}\left(\rho, w_{5}, w_{6}\right)=\sqrt{\frac{g_{t t} g_{x x}^{3} \rho^{6}}{\left(\rho^{2}+w_{5}^{2}+w_{6}^{2}\right)^{4}}} \\
& =\rho^{3} \frac{\left(\left(\rho^{2}+w_{5}^{2}+w_{6}^{2}\right)^{2}+w_{H}^{4}\right)\left(\left(\rho^{2}+w_{5}^{2}+w_{6}^{2}\right)^{2}-w_{H}^{4}\right)}{\left(\rho^{2}+w_{5}^{2}+w_{6}^{2}\right)^{4}} .
\end{aligned}
$$

With the ansatz $w_{5}=0$ and $w_{6}=w_{6}(\rho)$, the equation of motion becomes

$$
\begin{gathered}
\frac{\mathrm{d}}{\mathrm{d} \rho}\left[\mathcal{G}\left(\rho, w_{6}\right) \sqrt{\left.\frac{1}{1+\left(\frac{\mathrm{d} w_{6}}{\mathrm{~d} \rho}\right)^{2}} \frac{\mathrm{d} w_{6}}{\mathrm{~d} \rho}\right]}\right. \\
-\sqrt{1+\left(\frac{\mathrm{d} w_{6}}{\mathrm{~d} \rho}\right)^{2}} \frac{8 w_{H}^{8} \rho^{3} w_{6}}{\left(\rho^{2}+w_{6}^{2}\right)^{5}}=0 .
\end{gathered}
$$

The solutions of this equation determine the induced metric on the D7 brane which is given by

$$
\begin{aligned}
\mathrm{d} s^{2}= & \left(\tilde{w}^{2}+\frac{w_{H}^{4}}{\tilde{w}^{2}}\right) \mathrm{d} \vec{x}^{2}+\frac{\left(\tilde{w}^{4}-w_{H}^{4}\right)^{2}}{\tilde{w}^{2}\left(\tilde{w}^{4}+w_{H}^{4}\right)} \mathrm{d} t^{2} \\
& +\frac{1+\left(\partial_{\rho} w_{6}\right)^{2}}{\tilde{w}^{2}} \mathrm{~d} \rho^{2}+\frac{\rho^{2}}{\tilde{w}^{2}} \mathrm{~d} \Omega_{3}^{2}
\end{aligned}
$$

with $\tilde{w}^{2}=\rho^{2}+w_{6}^{2}(\rho)$. The D7 brane metric becomes $A d S_{5} \times S^{3}$ for $\rho \gg w_{H}, m$.

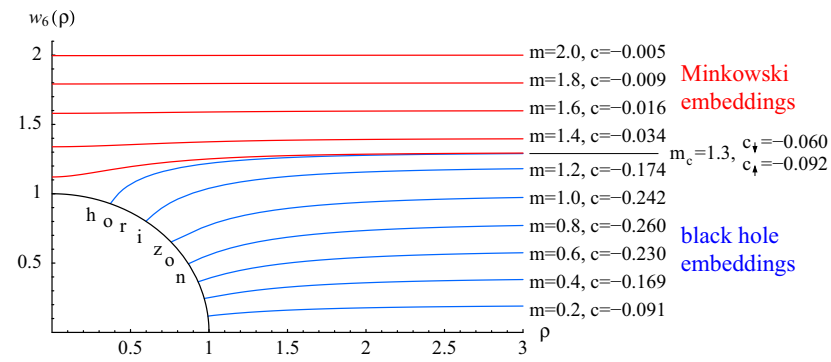

Fig. 25. Two classes of regular solutions in the AdS black-hole background. The quark mass $m_{q}$ is the parameter $m$ in units of $\Lambda \equiv \frac{w_{H}}{2 \pi \alpha^{\prime}}: m_{q}=m \Lambda$. We set $\Lambda=w_{H}=1$.

\subsubsection{First-order phase transition at finite temperature}

We now compute the explicit D7 brane solutions. The UV asymptotic (large $\rho$ ) solution, where the geometry returns to $A d S_{5} \times S^{5}$, is of the form

$$
w_{6}(\rho) \sim m+\frac{c}{\rho^{2}} .
$$

The parameters $m$ and $c$ have the interpretation as a quark mass and bilinear quark condensate $\langle\bar{\psi} \psi\rangle$, respectively, as discussed in sect. 6. These parameters can be taken as the boundary conditions for the second-order differential equation (7.8), which are solved using a numerical shooting technique. Of course the physical solutions should not have arbitrary $m$ and $c$. For a given value of $m, c$ is fixed by requiring regularity throughout the space.

The numerical solutions are illustrated in fig. 25 for several choices of $m$. We choose units such that the horizon is represented as a quarter circle with radius $w_{H}=1$.

As can be seen from the figure, there are two qualitatively different D7 brane embeddings. At large quark masses the D7 brane tension is stronger than the attractive force of the black hole. The D7 brane ends at a point outside the horizon, $\rho=0, w_{6} \geq w_{H}$, at which the $S^{3}$ wrapped by the D7 brane collapses (see (7.9)). Such a D7 brane solution is called a Minkowski embedding. They behave very similarly to the supersymmetric solutions in $A d S_{5} \times S^{5}$. As the mass decreases, there exists a critical value of the mass $m=m_{\text {crit }} \approx 0.92$ such that $w_{6}(\rho=0)=w_{H}$. For smaller masses the D7 brane is forced to fall into the black-hole horizon, i.e. the D7 brane ends at the horizon $w=w_{H}$ at which the $S^{1}$ of the blackhole geometry collapses. This is a so-called black-hole embedding.

From a geometrical point of view the two classes of embeddings differ by their topology: The D7 topology is $R^{3} \times$ $B^{4} \times S^{1}$ for Minkowski and $R^{3} \times S^{3} \times B^{2}$ for black-hole solutions. The appearance of a change in the topology of the embedding at $m_{\text {crit }}$ points to a phase transition in the dual field theory at exactly this critical value of the quark mass.

In fact, this embedding behaviour is a specific example of the more general problem of embedding a brane of arbitrary dimension in a black-hole geometry, as studied in [191]. Expanding the embedding equation near the horizon, it was shown that the equations have a self-similarity 

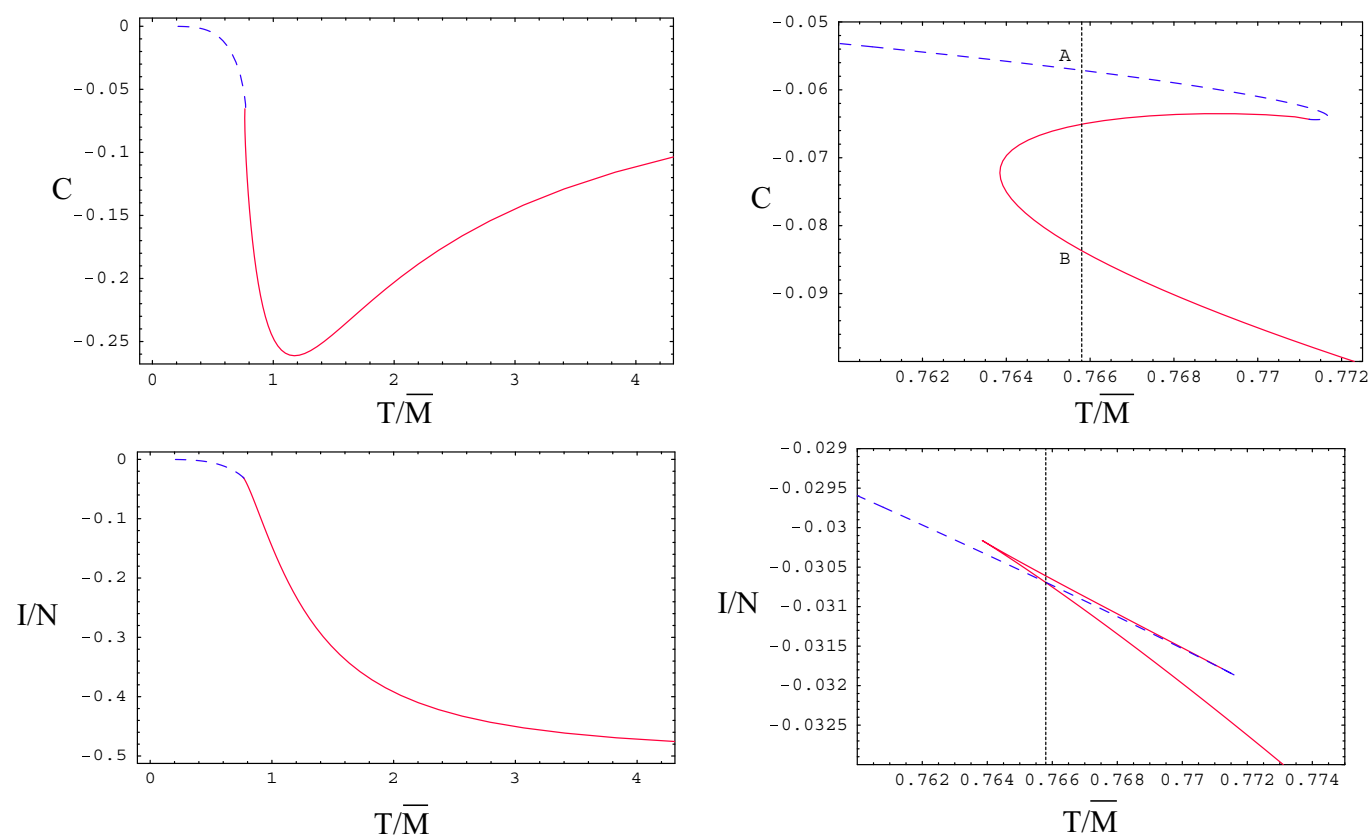

Fig. 26. Plots of the parameter $c$ vs. $1 / m \equiv T / \bar{M}$ for the regular solutions in AdS-Schwarzschild as given in [34]. A close-up of the transition point is also shown displaying the first-order transition. The action of the solutions is also plotted to determine the transition point. Note $\mathcal{N}$ is a normalization coefficient — see [34]. Figures kindly provided by D. Mateos, R. Myers and R. Thomson.

which implies that for a given range of $m$, there are an infinite number of embeddings.

The dependence of the condensate on the mass is illustrated in fig. 26. At $m=0$ the condensate $c$ is zero (the brane lies flat), so there is no chiral symmetry breaking in this gauge theory. As $m$ increases, the condensate $c$ initially increases and then decreases again. At sufficiently large $m$, the condensate becomes negligible, which is to be expected as the D7 brane ends in the region where the deformation of AdS is small. Recall that there is no condensate in the Yang-Mills theory with unbroken $\mathcal{N}=2$ supersymmetry described by D7 branes in un-deformed AdS. Once supersymmetry is broken by the temperature and the chiral symmetry is broken by the quark mass, it would be surprising if a condensate were not present though.

Since the D7 brane topology changes as $m_{\text {crit }}$ is crossed, one might expect a phase transition to occur at this point. Zooming in around $m_{\text {crit }}$, we see in fig. 26 that $c$ is multi-valued around the critical mass $m_{\text {crit }}$ as expected. This means that for a given quark mass in the regime $1.295 \leq m \leq 1.308$ there exist both Minkowski and blackhole embeddings. These solutions have the same quark mass $m$ but a different value of the quark condensate $c$.

The $c$ vs. $1 / m$ plot can also be considered as a plot of the condensate $c v s$. the temperature, since all dimensionful quantities are normalized by the temperature by setting $r_{H}=1$. For this we keep the quark mass $m$ fixed and vary the horizon $w_{H} \sim T$. Then, for small temperatures we recover the Minkowski embeddings, while for high temperatures we have black-hole embeddings. Heating up the system from zero temperature, we eventually reach a critical temperature $T_{\text {crit }}$ at which further supply of external energy does not increase the temperature of the system. It rather leads to the formation of a quark condensate. The jump in the quark condensate shows that the phase transition is discontinuous and thus of first order. The phase transition occurs in the deconfined phase of the field theory at a temperature $T_{\text {crit }}>T_{\text {deconf }}$.

\subsection{Mesons in the AdS black-hole background}

The true physical nature of the phase transition corresponding to the D7 branes switching from a Minkowski to a black-hole embedding is revealed through the behaviour of the mesons.

In the Minkowski phase, (i.e. when the $\mathrm{D} 7$ brane probe does not reach the black-hole horizon) the meson spectrum is similar to that in the zero-temperature theory. One can study perturbations of the D7 brane about the background embedding of the form $f(r) e^{-i w t}, w^{2}=M^{2}$ corresponding to stationary mesons. Requiring regularity for $f(r)$ determines the allowed meson masses $M$. Plots (taken from [34]) of the masses of the mesons associated with angular fluctuations in the $\left(w_{5}, w_{6}\right)$-plane and radial fluctuations in that plane are shown in fig. 27. As the mass approaches the critical value of $m$ the meson masses fall and the lowest radial mode becomes tachyonic.

If we now move to the other side of the transition, in the black-hole phase, when the D7 brane probe terminates on the horizon, the mesons become unstable and decay. In this case, there are no regular mesonic fluctuations with real masses. Instead the black hole supports quasi-normal 

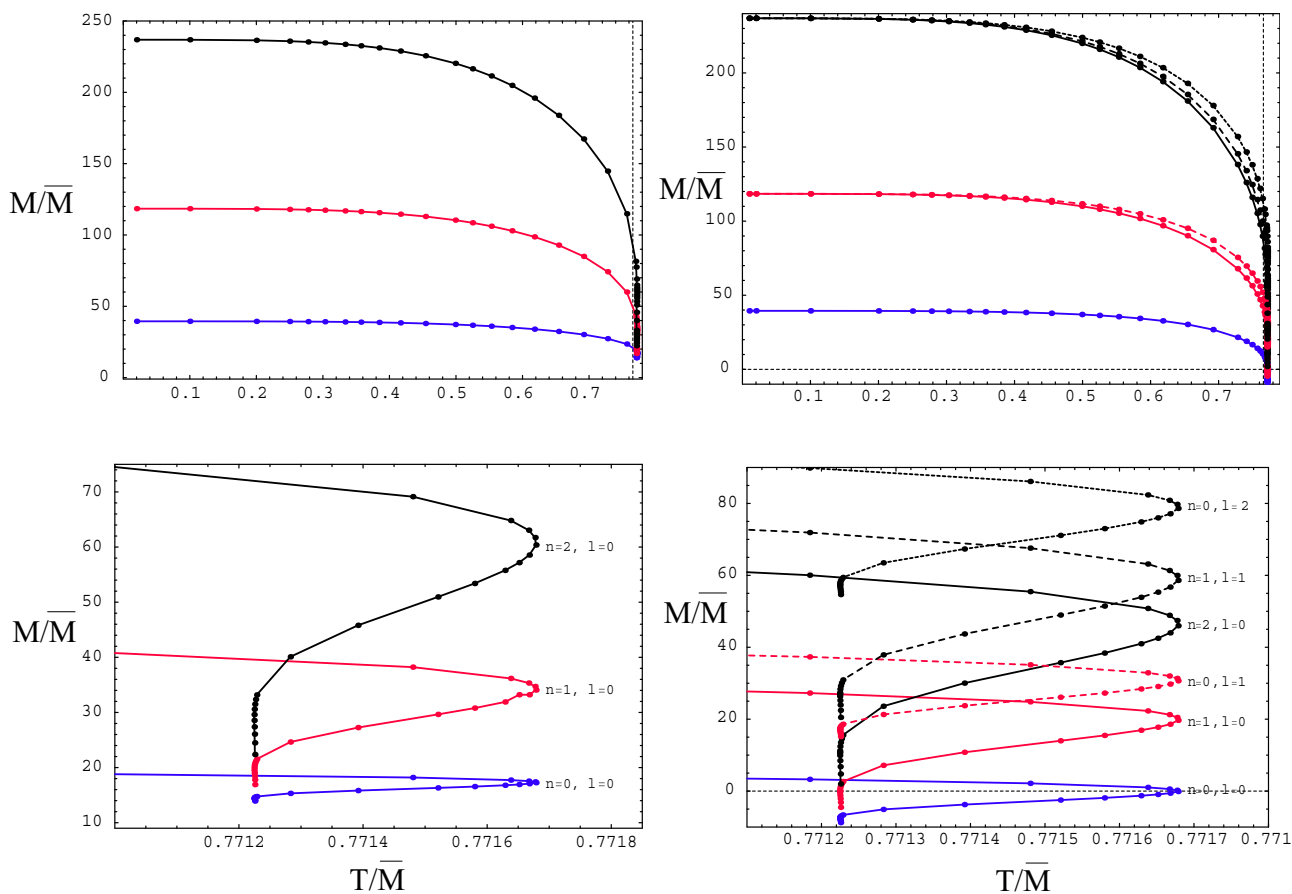

Fig. 27. Plots of meson masses in units of the quark mass vs. $1 / m \equiv T / \bar{M}$ for the Minkowski embeddings of the D7 brane as found in [34]. The figures on the left are for fluctuations of the D7 brane in the angular direction in the $\left(w_{5}, w_{6}\right)$-plane. Those on the right for radial fluctuations. In each case the lower figure is a close-up of the transition region. Figures kindly provided by D. Mateos, R. Myers and R. Thomson.

modes - fluctuations of the D7 that are purely infalling waves at the horizon. The mass that is extracted from these solutions is complex. The interpretation is that the mesons are not stable in the thermal plasma, and "melt" into it with a characteristic decay width given by the imaginary part of the quasi-normal eigenfrequency.

This is nicely described in [192]. An ansatz for D7 fluctuations of the form $f(\rho) e^{-(i \omega t+k \cdot x)}$ is again used. For the quasi-normal modes, the frequency $\omega$ develops a negative imaginary part, which provides a damping and corresponds to the decay width of the meson. The quasi-normal modes are eigenmodes with infalling boundary condition at the black-hole horizon.

In [192] the spectrum of scalar fluctuations of the D7 brane around its minimal-energy embedding was analyzed for a range of quark masses for fluctuations with zero spatial momentum. We linearize the equation of motion obtained from the DBI action for fluctuations of the D7 brane around the equilibrium configuration.

Consider embedding the D7 on the three-sphere within the five-sphere parameterized as

$$
\mathrm{d} \Omega_{5}^{2}=\mathrm{d} \theta^{2}+\sin ^{2} \theta \mathrm{d} \psi^{2}+\cos ^{2} \theta \mathrm{d} \Omega_{3}^{2} .
$$

In the special case of zero quark mass the D7 embedding is trivial, lying at $\theta=0$ for all $r$.

We consider fluctuations of the embedding in the $\theta$-direction of the form $\theta(r) e^{-i \omega t+i k \cdot x}$. Expanding the DBI action to quadratic order in $\theta$ leads to the eigenvalue equa- tion in the variable $z \equiv \frac{r_{H}}{r}$,

$$
\begin{aligned}
& \theta^{\prime \prime}-\frac{3+z^{2}}{z\left(1-z^{4}\right)} \theta^{\prime}+\frac{3}{z^{2}\left(1-z^{4}\right)} \theta+\frac{\Omega^{2}}{\left(1-z^{4}\right)^{2}} \theta \\
& -\frac{k^{2}}{\left(1-z^{4}\right)} \theta=0
\end{aligned}
$$

where $\Omega=\omega R^{2} / r_{H}$. In the UV (asymptotically AdS) limit $(z \rightarrow 0)$ the solution is a linear combination of $z^{1}$ and $z^{3}$. The latter is the normalizable mode and corresponds to a field theory quark bilinear via the AdS/CFT dictionary.

In the IR (near-horizon) limit $(z \rightarrow 1)$ the solution is a linear combination of $(1-z)^{+\frac{i \Omega}{4}}$ and $(1-z)^{-\frac{i \Omega}{4}}$. The solution with the negative exponent corresponds to a purely infalling wave.

In this case the eigenvalue problem can be solved using a method known in the GR literature as Leaver's method [193] to obtain the quasi-normal spectrum for $k=0$ (fig. 28).

For a non-zero quark mass the D7 embeddings are only known numerically and the analysis is much more involved. We need the solution to behave like a purely ingoing wave at the horizon and to be normalizable at infinity. The technique is to perform numerical integration of purely ingoing solutions outward from the horizon surface and normalizable solutions inward from infinity and attempt to match them smoothly at an intermediate value of the radial coordinate. This matching is only possible for a discrete set of frequencies which are the quasi-normal frequencies. An interesting picture is ob- 

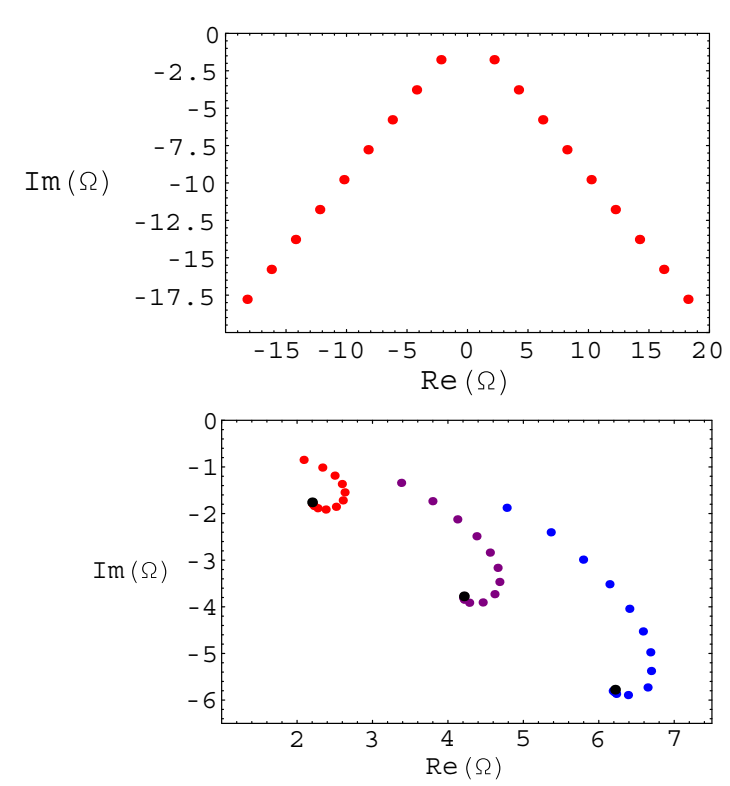

Fig. 28. The lowest quasi-normal modes for $m_{q}=0$ on the upper plot and the three lowest quasi-normal modes for increasing $m_{q}$ on the lower plot. The black points on the lower plot show the limiting values for $m_{q}=0$.

tained for the temperature dependence of quasi-normal modes, shown in fig. 28. As the embedding approaches the critical embedding the imaginary part of the quasi-normal frequencies is becoming smaller as one would expect -we are moving closer to the mesons being stable. The evolution of the quasi-normal modes at large $T$ into the stable mesons at small $T$ has been explicitly followed through the computation of the theory's spectral function in [194].

Computations involving semi-classical strings in the D3-D7 system have also been made. The properties of heavy-light mesons at finite temperature are determined in [195]. A long D3-D7 string describes a heavy deconfined quark and the energy loss and wake produced by such a string dragged through the plasma has been studied in [196-199].

Thus, the main physical characteristic of the phase transition is the mesons melting into the background thermal plasma. Note that since the temperature $T=$ $r_{H} /\left(R^{2} \pi\right)$ with $R=\lambda \alpha^{\prime 2}$ and the transition occurs when $m \sim r_{H}$, the temperature scale of the transition is

$$
T_{c} \sim \frac{m_{q}\left(2 \pi \alpha^{\prime}\right)}{\sqrt{\lambda} \alpha^{\prime} \pi} \sim \frac{2 m_{q}}{\sqrt{\lambda}} .
$$

The transition occurs at a temperature of roughly the meson mass.

\subsection{More thermodynamics}

The thermal transitions in the D3/D7 system compactified on an $S^{3}$ has been studied in [200]. The meson spectra in the presence of a black hole, whose radius is growing with time, have been computed in [201].

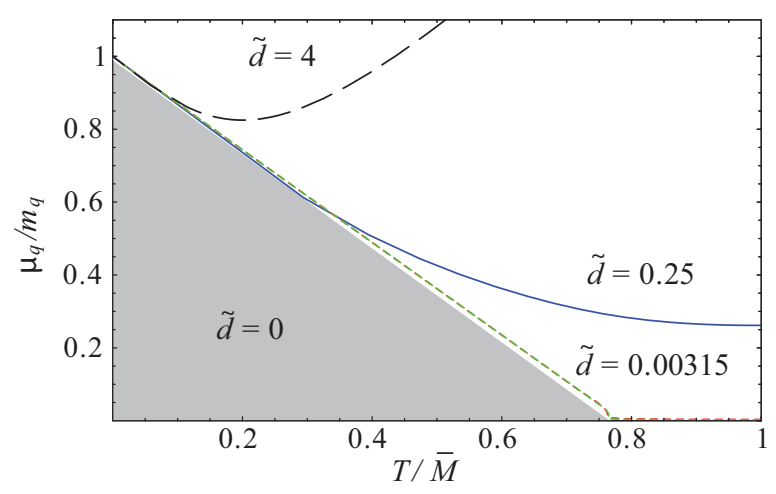

Fig. 29. Phase diagram for D7 branes in AdS-Schwarzschild at finite baryon density: the quark chemical potential $\mu_{q}$ divided by the quark mass is plotted $v s$. the temperature $T$ divided by $\bar{M}=2 m_{q} / \sqrt{\bar{\lambda}}$. Two different regions are displayed: The shaded region with vanishing baryon density and the white region with finite baryon density. The multi-valued region at the lower tip of the transition line is not resolved here. The curves are lines of equal baryon density. The curve for the critical density $\tilde{d}^{*}=$ 0.00315 displays where the first-order phase transition between two black-hole embeddings disappears. Figure by M. Kaminski and F. Rust.

In the presence of a finite quark or isospin density, introduced through a vev for the time component of the gauge field on the probe brane, the structure of the phase diagram becomes more involved. In particular, there are unstable regions in the phase diagrams. Studies of finite chemical potential and finite density effects for D7 brane probes in the AdS-Schwarzschild background may be found in [101,202-210].

We also note that equivalent phase transitions to those above occur in the D4/D6 system of sect. 6.4 (see [21] for the details and [35] for related work).

Considerable work has also been done on the SakaiSugimoto model (see sect. 6.5 above) at finite temperature. That model also displays a first-order meson melting transition as described in $[195,211]$. Additional finite density studies can be found in $[173,174,212-217]$. As an example we consider here spectral functions at finite temperature and the quark chemical potential as discussed in [209]. The phase diagram was found in $[202,205]$ and is displayed in fig. 29. In the grey-shaded area, the baryon density $n_{B}$ is zero, the first-order phase transition between Minkowski and black-hole embeddings occurs. In the white area, the baryon density is non-zero. In this region, only black-hole embeddings are stable. Lines of constant baryon density are displayed in colour. For small non-zero values of the baryon density, a first-order transition between two black-hole embeddings occurs, which disappears above a critical value for the quark density given by $\tilde{d}^{*} \equiv 2^{5 / 2} n_{B} /\left(N_{f} \sqrt{\lambda} T^{3}\right)=0.00315$, with $n_{B}$ the baryon density. Moreover, there is a multi-valued region at the bottom of the separation line between the greyshaded and the white region, which is not resolved here. According to the phase diagram, within the black-hole phase (i.e. in the white region) for fixed quark mass, there is a temperature-dominated region for large temperatures 

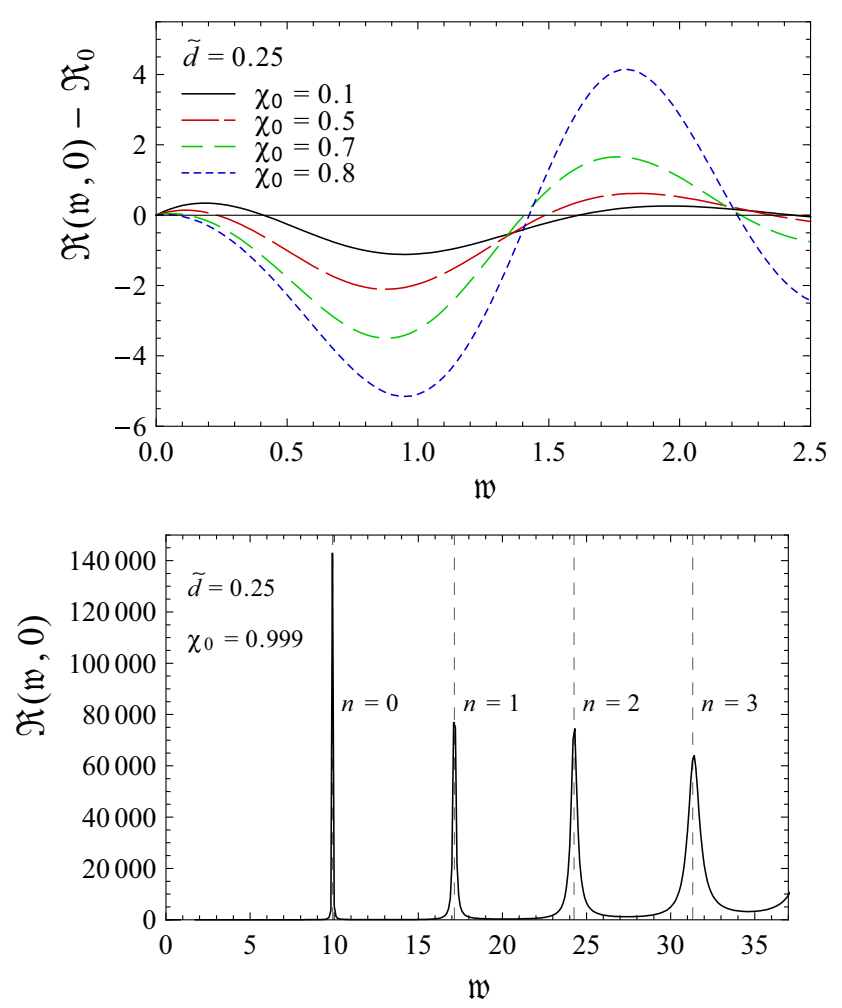

Fig. 30. The finite-temperature part of the spectral function $\mathfrak{R}-\mathfrak{R}_{0}$ (in units of $N_{f} N T^{2} / 4$ ) in the temperature-dominated region (upper plot) and in the potential-dominated region (lower plot). $\tilde{d}$ parametrizes the quark density and $\chi_{0}$, introduced in [202], essentially corresponds to $m_{q} / T$. Figures from [209].

to the far right, and a potential-dominated region for small temperatures to the left. In the two regions, the spectral functions show a qualitatively different behaviour. We consider the spectral functions for the current-current correlator coupling to the gauge field on the D7 brane. The result is displayed in fig. 30. In the temperature-dominated region, the spectral function, i.e. the imaginary part of the retarded Green function, displays very broad peaks corresponding to unstable vector mesons. This is shown in the upper plot of the figure. In the potential-dominated region however, the peaks become very narrow and their location coincides exactly with the supersymmetric meson spectrum discussed earlier in sect. 3.19 (by supersymmetry, the scalar and vector spectra coincide).

A further interesting point is that the location of the peaks first moves to lower frequencies when the temperature is decreased until they reach a minimum. When decreasing the temperature further, the peaks move the larger frequencies again, while becoming narrower. This corresponds to a movement of the poles similar to the one displayed in fig. 28.

\subsection{Mesons from D7 branes with external B fields}

Supersymmetric versions of embeddings in backgrounds with $B$ field have been presented above in sect. 5.2 . There

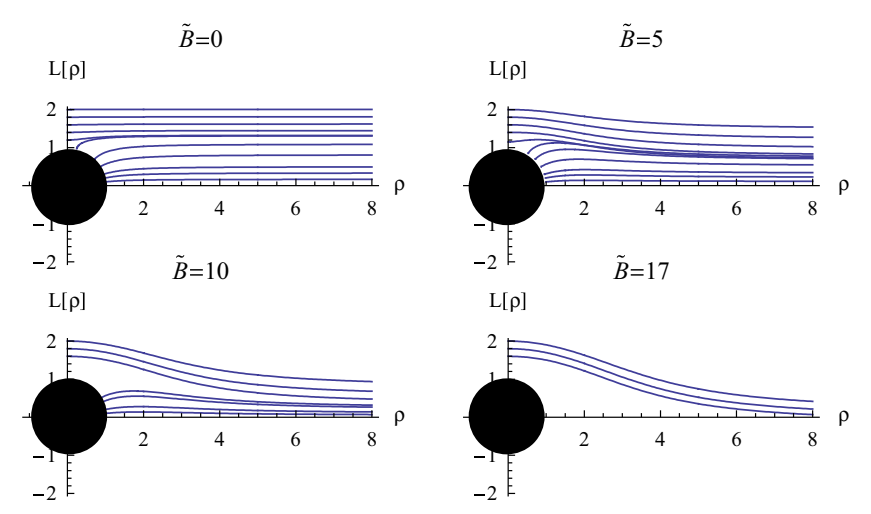

Fig. 31. Embedding function $L$ as a function of the radial coordinate $\rho$ for D7 branes embedded in the black-hole background with external magnetic fields, for different values of the normalized (dimensionless) external field $\tilde{B}$. Increasing values of $\tilde{B}$ for fixed $T$ show the repulsive nature of the magnetic $B$ field, which is switched on in two spatial directions parallel to the boundary. We see that for large enough $\tilde{B}$, the black-hole phase is never reached, and spontaneous chiral symmetry breaking occurs. From [219].

are also interesting effects in non-supersymmetric backgrounds with $B$ fields.

A Zeeman splitting is observed if a pure gauge-external $B$ field is turned on in two spatial directions parallel to the AdS boundary [23,218]. Such a $B$ field breaks supersymmetry completely. As shown in [23,218], it induces spontaneous chiral symmetry breaking and Goldstone bosons by virtue of a similar mechanism as discussed in sect. 6 above.

As discussed in sect. 7.1, there is no spontaneous chiral symmetry breaking in the finite-temperature field theory dual of the AdS-Schwarzschild black-hole background. However, if a $B$ field of the form of [23] of sufficient strength is switched on, the chiral symmetry-breaking mechanism induced by this $B$ field dominates and is present even in the black-hole background [219,220]. This is shown in fig. 31 .

With an external electric field, i.e. a $B$ field turned on in the temporal and one spatial direction parallel to the boundary, a meson mass shift similar to the Stark effect arises [219]. In this case, there is an attraction of the D7 brane probes towards the origin and no chiral symmetry breaking occurs $[219,221]$.

\subsection{Summary}

We have seen that the AdS/CFT correspondence implies the existence of a novel thermal phase transition in theories with quarks. As the temperature passes through the scale of the meson mass, there is a first-order phase transition with a small jump in the value of the quark condensate. The mesons of the theory melt into the thermal bath at this scale. Note that this transition is distinct from the deconfinement transition of the glue-fields. Lattice calculations [188-190] do not reveal such a first-order transition in QCD, so it is probably that it is an artifact 
of the large- $N$ regime. Meson melting does occur in QCD though and it is promising that we have a theoretical tool to address that process. The mesons of this theory are tightly bound and so harder to dissociate than those in QCD - heavy-heavy mesons in QCD are not expected to survive to as high a temperature as their mass scale as we see here.

\section{AdS/QCD}

Inspired by holography a number of authors have proposed phenomenological models of QCD generically called AdS/QCD. These models consist of a gauge theory in a curved space (usually AdS) with the field content picked to holographically match to certain QCD bound states and operators. This sort of modelling is necessarily a leap in the dark. From the string theory side one might expect that as one approached QCD from the theories at infinite 't Hooft coupling, string corrections would become large - one should be working in a string theory and not in a field theory. Nevertheless, the string models described above contain confinement and chiral symmetry breaking and the ratio of meson masses do appear to match the QCD values to a few 10\% (the absolute values do not match unless one extrapolates to order-one 't Hooft coupling - in the string models these states are tightly bound with mass $\sim m_{q} / \sqrt{g_{Y M}^{2} N}$ ). It is therefore interesting to try model building in the spirit of these models.

\subsection{A simple model}

We will concentrate on the simplest example of this sort proposed in [222] and [223,224] which is closest in spirit to the string models in sects. $6.2-6.4$ (a phenomenological model in the spirit of sect. 6.5 can be found in [225]).

The field theory will live in an AdS space in five dimensions (discarding the extra five dimensions of the string theory removes the $S O(6)$ global symmetry of the $\mathcal{N}=4$ model as would happen were the superpartners to be decoupled),

$$
\mathrm{d} s^{2}=r^{2} \mathrm{~d} x_{4}^{2}+\frac{\mathrm{d} r^{2}}{r^{2}} .
$$

The radial coordinate $r$ will be interpreted as the holographic energy scale of the theory (see (3.68)). As written the metric has an $S O(2,4)$ symmetry and would appear to describe a conformal gauge background. To break that symmetry and impose confinement a crude, hard wall is imposed at $r=r_{0}$ - the theory will only live at $r \geq r_{0}$. One can think of this scale as the mass gap of the gauge background.

We will choose to describe the quark mass and condensate and the pion fields in the model. We introduce a scalar field

$$
X=X_{0} e^{2 i \pi^{a} t^{a}} .
$$

$X_{0}$ will be a background field that describes the quark mass and condensate (these are both assumed to be matrices in flavour space that are proportional to the identity). As we saw in (2.15) for a scalar to describe a quark bilinear operator $(\Delta=3)$ it must have mass squared $m^{2}=\Delta(\Delta-4)=-3$ in AdS and then the solution is of the form

$$
X_{0}=\frac{1}{2} \frac{m}{r}+\frac{1}{2} \frac{\Sigma}{r^{3}} .
$$

Remember that $r$ has energy dimension so $m$ is the mass and $\Sigma$ the condensate. $\pi^{a}$ are then the $N_{f}^{2}-1$ pion fields.

In addition, the model describes the vector and axial vector states through two massless gauge fields dual to the operators $\bar{q}_{L} \gamma^{\mu} q_{L}$ and $\bar{q}_{R} \gamma^{\mu} q_{R}{ }^{10}$. The action is

$$
S=\int_{r_{0}}^{\infty} \mathrm{d}^{5} x \sqrt{-g} \operatorname{Tr}\left\{|D X|^{2}+3|X|^{2}-\frac{1}{4 g_{5}^{2}}\left(F_{L}^{2}+F_{R}^{2}\right)\right\},
$$

where $X$ transforms on the left under $S U\left(N_{f}\right)_{L}$ and on the right under $S U\left(N_{f}\right)_{R}$.

It is of course completely ad hoc to only describe these states. In QCD there are many other states with mass of order the $a_{0}$ and the $\rho$ but we simply choose to ignore them.

The mass, condensate and the position of the hard wall will be parameters of the theory that are fit. There is also $g_{5}$, which in string theory duals is a prediction in terms of the gauge theory 't Hooft coupling $g_{Y M}^{2} N$. In the phenomenological approach though, this relation is abandoned and the value of $g_{5}$ is fitted to the vector current correlator extracted from QCD,

$$
\int \mathrm{d}^{4} x e^{i q x}\left\langle J_{\mu}^{a}(x) J_{\nu}^{b}(0)\right\rangle=\delta^{a b}\left(q_{\mu} q_{\nu}-q g_{\mu \nu}\right) \Pi_{V}\left(-q^{2}\right)
$$

where $J_{\mu}^{a}(x)=\bar{q} \gamma_{\mu} T^{a} q$. For QCD, the leading-order contribution to $\Pi_{V}\left(-q^{2}\right)$ is $[226]$

$$
\Pi_{V}\left(-q^{2}\right)=-\frac{N}{24 \pi^{2}} \ln \left(-q^{2}\right)
$$

In order to calculate this quantity from the fivedimensional model, we appeal to the AdS/CFT correspondence. The five-dimensional vector field $V_{\mu}^{a}(x, r)=$ $\left(A_{L \mu}^{a}(x, r)+A_{R \mu}^{a}(x, r)\right) / 2$ acts as a source for the fourdimensional vector current $J_{\mu}^{a}(x)$ in the limit $r \rightarrow \infty$. It obeys the equation of motion

$$
\partial_{\mu}\left(\frac{1}{g_{5}^{2}} e^{\phi} \sqrt{-g} g^{\mu \alpha} g^{\nu \beta}\left(\partial_{\alpha} V_{\beta}^{a}-\partial_{\beta} V_{\alpha}^{a}\right)\right)=0 .
$$

We look for solutions of the form $V^{\mu}(x, r)=V_{0}^{\mu}(x) v(x, r)$, with $\lim _{r \rightarrow \infty} v(x, r)=1$, so that $V_{0}^{\mu}(x)$ will act as a source of dimension one for $J_{\mu}^{a}(x)$. Solving the equation of motion (8.7) in the $V^{r}(x, r)=0$ gauge gives

$$
v(q, r)=-\frac{\pi}{2} \mathcal{Y}_{1}(q / r) \sim 1-\frac{q^{2}}{4 r^{2}} \ln \left(\frac{-q^{2}}{r^{2}}\right), \quad \text { as } r \rightarrow \infty,
$$

where $\mathcal{Y}_{1}$ is a Bessel function of the second kind. Substituting the solution back into the action and differentiating

\footnotetext{
10 The mass-conformal dimension relation for vector operators is $m^{2}=(\Delta-1)(\Delta-3)$, thus $m^{2}=0$.
} 
Table 4. Results for meson variables in AdS/QCD. AdS A is the best fit to the starred variables. Model B is the best fit to all the observables.

\begin{tabular}{|c|c|c|c|}
\hline Observable & $\begin{array}{c}\text { Measured } \\
(\mathrm{MeV})\end{array}$ & $\begin{array}{c}\text { AdS A } \\
(\mathrm{MeV})\end{array}$ & $\begin{array}{c}\text { AdS B } \\
(\mathrm{MeV})\end{array}$ \\
\hline$m_{\pi}$ & $139.6 \pm 0.0004$ & $139.6^{*}$ & 141 \\
$m_{\rho}$ & $775.8 \pm 0.5$ & $775.8^{*}$ & 832 \\
$m_{a_{1}}$ & $1230 \pm 40$ & 1363 & 1220 \\
$f_{\pi}$ & $92.4 \pm 0.35$ & $92.4^{*}$ & 84.0 \\
$F_{\rho}^{1 / 2}$ & $345 \pm 8$ & 329 & 353 \\
$F_{a_{1}}^{1 / 2}$ & $433 \pm 13$ & 486 & 440 \\
\hline
\end{tabular}

twice with respect to the source $V_{0}^{\mu}$ gives the vector current correlator

$$
\Pi_{V}\left(-q^{2}\right)=\left[\frac{1}{g_{5}^{2} q^{2}} r^{3} \partial_{r} v(q, r)\right]_{r=\infty},
$$

which (up to contact terms) yields

$$
\Pi_{V}\left(-q^{2}\right)=-\frac{1}{2 g_{5}^{2}} \ln \left(-q^{2}\right) .
$$

Finally, comparing this to the perturbative QCD result (8.6) determines the five-dimensional coupling to be

$$
g_{5}^{2}=\frac{12 \pi^{2}}{N}
$$

It may appear rather surprising to be fitting to the asymptotic perturbative result when a gravity dual is inherently a description of a strongly coupled gauge theory. The argument that is usually made is that perturbative QCD is conformal in the UV and so it is natural to match to the UV behaviour in AdS which is also conformal. One captures this conformality in the model if not the asymptotically free running of the coupling.

Now as usual one can solve (8.7) for solutions of the form $V=V(r) e^{i p . x}, p^{2}=-M^{2}$ with $V(r)$ falling to zero as $r \rightarrow \infty$. One must choose a (necessarily arbitrary) boundary condition at the hard wall and we can for example choose $\partial_{r} V=0$. We can therefore extract the masses of the $\rho$ and its excited states.

One can also extract the decay constant for a $\rho$ decaying to a photon. One integrates the action by parts treating one field $V$ as a solution of the equation of motion and one as a background external field. The coupling is then

$$
F_{\rho}^{2}=\frac{1}{g_{5}^{2}} V_{\rho}^{\prime \prime}(r \rightarrow \infty) .
$$

Similarly, one can study the axial vector gauge field and the pion to determine the pion mass, $a_{1}$ mass and their decay constants. The best fit results to the QCD data are shown in table 4 . There is a good fit to the data.

\subsection{Higher-order pion interactions}

The chiral symmetry-breaking pattern of AdS/QCD means that the pions necessarily take the form of a chiral
Lagrangian model. In this formalism the coefficients are a prediction though. In [223] the order $p^{4}$ terms in the chiral Lagrangian were estimated in the simplest AdS/QCD model (assuming the lightest rho dominated these terms). These terms take the form

$$
\begin{aligned}
& \mathcal{L}_{4}=L_{1} \operatorname{Tr}^{2}\left[D_{\mu} U^{\dagger} D^{\mu} U\right]+L_{2} \operatorname{Tr}\left[D_{\mu} U^{\dagger} D_{\nu} U\right] \\
& \quad \times \operatorname{Tr}\left[D^{\mu} U^{\dagger} D^{\nu} U\right]+L_{3} \operatorname{Tr}\left[D_{\mu} U^{\dagger} D^{\mu} U D_{\nu} U^{\dagger} D^{\nu} U\right] \\
& +L_{4} \operatorname{Tr}\left[D_{\mu} U^{\dagger} D^{\mu} U\right] \operatorname{Tr}\left[U^{\dagger} \chi+\chi^{\dagger} U\right] \\
& +L_{5} \operatorname{Tr}\left[D_{\mu} U^{\dagger} D^{\mu} U\left(U^{\dagger} \chi+\chi^{\dagger} U\right)\right] \\
& +L_{6} \operatorname{Tr}^{2}\left[U^{\dagger} \chi+\chi^{\dagger} U\right]+L_{7} \operatorname{Tr}^{2}\left[U^{\dagger} \chi-\chi^{\dagger} U\right] \\
& +L_{8} \operatorname{Tr}\left[\chi^{\dagger} U \chi^{\dagger} U+U^{\dagger} \chi U^{\dagger} \chi\right] \\
& \quad i L_{9} \operatorname{Tr}\left[F_{R}^{\mu \nu} D_{\mu} U D_{\nu} U^{\dagger}+F_{L}^{\mu \nu} D_{\mu} U^{\dagger} D_{\nu} U\right] \\
& +L_{10} \operatorname{Tr}\left[U^{\dagger} F_{R}^{\mu \nu} U F_{L \mu \nu}\right] .
\end{aligned}
$$

We reproduce the results from [223] in the following table:

\begin{tabular}{ccc}
\hline & Experiment & $A d S_{5}$ \\
\hline$L_{1}$ & $0.4 \pm 0.3$ & 0.4 \\
$L_{2}$ & $1.4 \pm 0.3$ & 0.9 \\
$L_{3}$ & $-3.5 \pm 1.1$ & -2.6 \\
$L_{4}$ & $-0.3 \pm 0.5$ & 0.0 \\
$L_{5}$ & $1.4 \pm 0.5$ & 1.7 \\
$L_{6}$ & $-0.2 \pm 0.3$ & 0.0 \\
$L_{9}$ & $6.9 \pm 0.7$ & 5.4 \\
$L_{10}$ & $-5.5 \pm 0.7$ & -5.5 \\
\hline
\end{tabular}

\subsection{Glueballs}

It is also possible to include glueballs into AdS/QCD [227229 ] through additional scalars in the bulk. We can associate the $0^{++}$glueballs with the operator $\operatorname{Tr} F^{2}$ a dimension-4 operator - the usual AdS dictionary teaches that the dual supergravity field should be massless. The equation of motion (for a solution of the form $\phi=$ $\left.\phi(r) e^{i p x}, p^{2}=-M^{2}\right)$ is

$$
\left(\frac{1}{r} \partial_{r} r^{5} \partial_{r}+M^{2}\right) \phi(r)=0 .
$$

If we again impose Neumann boundary conditions $\left(\partial_{z} \phi=0\right)$ at the hard wall we find the glueball masses (normalizing to the lattice gauge theory [230,231] value for the lightest mass state) $M_{1}=1.63 \mathrm{GeV}, M_{2}=2.98 \mathrm{GeV}$, $M_{3}=4.33 \mathrm{GeV}$, etc.

\subsection{A plethora of AdS/QCD phenomenology}

A considerable number of other aspects of QCD phenomenology have been successfully addressed using AdS/QCD which we cannot completely review here. The reader is referred to the following references. Strange quarks are added in [232]. Higher-spin mesons are studied in [233]. 
Baryon states are included in [234]. Four-point currentcurrent correlators relevant to the $\Delta I=1 / 2$ rule and the $B_{K}$ parameter for $K$-meson mixing are analyzed in [235]. Heavy-quark potentials are computed in [236]. The AdS/QCD model is related to light-cone QCD in [237, 238] allowing form factor computations. Form factors for mesons are also in $[239,240]$.

Properties of QCD at high temperature and density and the deconfinement transition have been analyzed in this context in [241-247].

Such models have also been adapted to describe walking [248] technicolour [249,250] dynamics for electroweak symmetry breaking in [251-255]. It is worth remarking that very similar ideas to these models have inspired the field of Higgsless electroweak models [256, 257] and their deconstructed $[258,259]$ partners for example in [260].

\subsection{Regge behaviour and the soft wall}

The basic AdS/QCD model does not have the expected Regge behaviour for the towers of radially excited states $\left(M_{n}^{2} \sim n\right)$ [261-264]. To see this, consider the action for the gauge field in AdS describing the rho mesons,

$$
I \sim \int \mathrm{d}^{5} x e^{-\Phi(z)} \sqrt{-g} F^{2} .
$$

Here we have included a dilaton field $\Phi$ that is a constant in the basic AdS/QCD model. The equation of motion for a solution of the form $A_{x}=f(z) e^{i k x}, k^{2}=M^{2}$ is

$$
\left(r \partial_{r} r^{3} \partial_{r}+M^{2}\right) f=0 .
$$

Changing variables to $z=1 / r$ and substituting

$$
f=e^{B / 2} \psi, \quad B=\phi+\ln r,
$$

we find

$$
-\psi^{\prime \prime}+V(z) \psi=M^{2} \psi, \quad V=\frac{1}{4}\left(B^{\prime}\right)^{2}-\frac{1}{2} B^{\prime \prime},
$$

which is of a Schrödinger equation form.

If we impose the IR boundary by putting in a hard cut-off then the Schrödinger potential in the IR is that of a square well. The mass spectrum therefore grows as $M_{n}^{2} \sim n^{2}$, in contradiction with the physically observed Regge behaviour.

One might simply argue that this is a sign that the supergravity approximation is breaking down when we try to apply these methods to QCD — string theory naturally gives Regge behaviour, so a resolution would be to work with a full string theory. In [265] it was pointed out though that if the dilaton grows as $\frac{1}{r^{2}}$ in the IR the potential $V$ will be of the form

$$
V=z^{2}+\frac{3}{4 z^{2}}
$$

The exact solution is known and $M_{n}^{2}=4(n+1)$. Regge style behaviour is therefore accessible in principle in the supergravity regime. None of this behaviour is derivable though merely posited.

\subsection{Improvement and perfection}

An obvious criticism of AdS/QCD is that it is a model rather than being derived explicitly from the QCD Lagrangian. There is no understanding of systematic errors. Can we hope to improve the model then?

Presumably, in reality, the weakly coupled string theory model will only be valid in the strong-coupling regime of QCD at low energies. It is therefore a low-energy effective theory. An obvious consequence of this is that a UV cut-off should be imposed [266,267] and the scaling dimension of operators, values of higher-dimension operator couplings and expectation values of operators should all be matched at the cut-off. In principle, this is possible although there is no obvious truncation to a finite number of such matchings and the resulting model need not be weakly coupled.

The introduction of expectation values for relevant operators is discussed and introduced in [268-271]. Instanton effects are included in [267]. In [272,273] backreacted geometries in five-dimensional non-critical string theory are generated that have a dilaton profile set to match the QCD running coupling - the models display confinement and chiral symmetry breaking (through the addition of D4 and $\overline{\mathrm{D} 4}$ branes). Meson properties have not yet been computed there though. The inclusion of higher-dimension operator couplings is discussed in $[266,274]$. Improvements to the phenomenological fit can be achieved by all these methods although at the expense of additional free parameters.

The basic AdS/QCD model also inputs chiral symmetry breaking and the quark mass through two independent parameters $c$ and $m$. In reality, the quark condensate should be a prediction of the background gauge dynamics (the metric) and the value of $m$. The more complete string models of chiral symmetry breaking discussed above in sects. 6.2-6.4 do contain this explicit dynamics. In [275] the dilaton flow model of chiral symmetry breaking was adapted to an AdS/QCD model keeping that dynamical behaviour. The computations are essentially those of sect. 6.2 but with $g_{5}$ fixed as in eq. (8.11). The resulting model has one fewer free parameter and gives a match to the data of similar quality to the basic AdS/QCD model.

Finally, higher-dimension operators in the gravity dual's action, representing stringy corrections, have been included in [276].

\subsection{Summary}

AdS/QCD is a tidy crystallization of the ideas of holographic chiral symmetry-breaking models applied to QCD. Generically, such models do well at reproducing QCD phenomenology at the $10 \%$ level, suggesting that their parent string theory models are capturing crucial aspects of QCD dynamics. It remains a challenge though both to understand how to systematically move towards a complete description of QCD, and how to precisely embed the AdS/QCD models into string theory. 


\section{Conclusion}

In this review we have seen how a new theoretical technique for calculating in strongly coupled gauge theory has emerged from string theory. The AdS/CFT correspondence in its initial form described the highly supersymmetric and conformal $\mathcal{N}=4$ Yang-Mills theory at large 't Hooft coupling. Deformations of the gravity dual have since led to the understanding of confinement in nonconformal theories. Here, we have concentrated on the next step necessary for moving towards QCD, which consists of adding quarks in the fundamental representation. The strong dynamics of the gauge fields bind the quarks into tightly bound mesonic states. When quarks are included in non-supersymmetric geometries, we have seen that chiral symmetry breaking is generated - the quarks acquire a dynamical mass and there are Goldstone fields associated with the symmetry breaking, the analogues of the pions. It is very pleasing that the examples presented show a large number of phenomena we observe in QCD.

The gravity dual description also works well for strongly coupled finite-temperature field theories, for example, for describing dynamical processes such as diffusion and meson melting. These results are potentially relevant for the quark-gluon plasma, for which standard perturbative or lattice gauge theory methods are not easily available.

Given these qualitative successes, it has been tempting to make quantitative comparisons to QCD. This necessarily involves ignoring the absence of asymptotic freedom and the presence of massive, but not decoupled, superpartners. The 't Hooft coupling is also brought down from the infinite coupling limit to make these comparisons. Surprisingly though, such comparisons do hold up well (typically at the $10 \%$ level). This suggests that a wide range of gauge theories share a number of even quantitative properties. There is considerable hope that calculations relevant to QCD can be performed. This hope must be tempered though by the difficulties of bringing systematic errors under control.

We want to stress though that the value of the gravity dual approach are of a more principal nature. They provide an opportunity for new exchanges between string theory and quantum field theory, which leads to a fresh look at both fields. This has lead to progress in both areas. Moreover, in this context, string theory as a candidate for a unified theory of fundamental interactions has made a significant step towards a more applied approach of being applicable to experimentally testable models.

\section{Other reviews}

A number of other recent reviews may be of interest to our readers. The "classic" review of the AdS/CFT correspondence is [277]. Reference [278] contains a description of generalizations to theories with broken conformal invariance. Reference [279] covers material on D7 brane probes in supersymmetric theories. The Sakai-Sugimoto model is reviewed in [280]. Thermal properties of these theories are reviewed in [281].
We would like to thank our co-authors with whom we have worked on some of the topics described over the last years: Riccardo Apreda, James Babington, Johannes Große, Kazuo Ghoroku, Zachary Guralnik, Matthias Kaminski, Dieter Lüst, René Meyer, Felix Rust, Jonathan Shock, Christoph Sieg, Andrew Tedder, Diana Vaman and Tom Waterson. We would like to thank Martin Ammon, Dietmar Ebert and Kasper Peeters for a critical reading of the manuscript, as well as Daniel Arean, Gunnar Bali, Biagio Lucini and Alfonso Ramallo for comments. J.E. is grateful to the Isaac Newton Institute, Cambridge, for hospitality in August 2007, where part of this review was written.

\section{References}

1. B. Zwiebach, A First Course in String Theory (University Press, Cambridge, UK, 2004) p. 558.

2. E. Kiritsis, String Theory in a Nutshell (University Press, Princeton, USA, 2007) p. 588.

3. K. Becker, M. Becker, J.H. Schwarz, String Theory and M-theory: A Modern Introduction (Cambridge University Press, Cambridge, UK, 2007) p. 739.

4. M.B. Green, J.H. Schwarz, E. Witten, Superstring Theory, Cambridge Monogr. Math. Phys., Vol. 1: Introduction (University Press, Cambridge, UK, 1987) p. 469.

5. J. Polchinski, String Theory, Vol. 1: An introduction to the bosonic string (University Press, Cambridge, UK, 1998) p. 402.

6. D.J. Gross, F. Wilczek, Ultraviolet behavior of nonabelian gauge theories, Phys. Rev. Lett. 30, 1343 (1973).

7. H.D. Politzer, Reliable perturbative results for strong interactions? Phys. Rev. Lett. 30, 1346 (1973).

8. G.'t Hooft, A planar diagram theory for strong interactions, Nucl. Phys. B 72, 461 (1974).

9. T. DeGrand, C. DeTar, Lattice Methods for Quantum Chromodynamics (World Scientific, NJ, 2006) p. 345.

10. J. Smit, Introduction to quantum fields on a lattice: A robust mate, Cambridge Lect. Notes Phys. 15, 1 (2002).

11. M. Creutz, Quarks, Gluons and Lattices, Cambridge Monogr. Math. Phys. (University Press, Cambridge, UK, 1983) p. 169.

12. J. Polchinski, Dirichlet-Branes and Ramond-Ramond Charges, Phys. Rev. Lett. 75, 4724 (1995) arXiv:hepth/9510017.

13. J. Polchinski, Lectures on D-branes, arXiv:hepth/9611050.

14. C.V. Johnson, D-branes (University Press, Cambridge, UK, 2003) p. 548.

15. J.M. Maldacena, The large $N$ limit of superconformal field theories and supergravity, Adv. Theor. Math. Phys. 2, 231 (1998) arXiv:hep-th/9711200.

16. A. Karch, E. Katz, Adding flavor to AdS/CFT, JHEP 06, 043 (2002) arXiv:hep-th/0205236.

17. M. Kruczenski, D. Mateos, R.C. Myers, D.J. Winters, Meson spectroscopy in AdS/CFT with flavour, JHEP 07, 049 (2003) arXiv:hep-th/0304032.

18. J. Babington, J. Erdmenger, N.J. Evans, Z. Guralnik, I. Kirsch, Chiral symmetry breaking and pions in nonsupersymmetric gauge/gravity duals, Phys. Rev. D 69, 066007 (2004) arXiv:hep-th/0306018.

19. G. Bali, F. Bursa, Meson masses at large $N_{c}$, arXiv: 0708.3427 [hep-lat]. 
20. L. Del Debbio, B. Lucini, A. Patella, C. Pica, Quenched mesonic spectrum at large $N$, arXiv:0712.3036 [hep-th].

21. M. Kruczenski, D. Mateos, R.C. Myers, D.J. Winters, Towards a holographic dual of large-N(c) QCD, JHEP 05, 041 (2004) arXiv:hep-th/0311270.

22. K. Ghoroku, M. Ishihara, A. Nakamura, Flavor quarks in AdS (4) and gauge/gravity correspondence, Phys. Rev. D 75, 046005 (2007), arXiv:hep-th/0612244.

23. V.G. Filev, C.V. Johnson, R.C. Rashkov, K.S. Viswanathan, Flavoured large $N$ gauge theory in an external magnetic field, arXiv:hep-th/0701001.

24. T. Sakai, S. Sugimoto, Low energy hadron physics in holographic QCD, Prog. Theor. Phys. 113, 843 (2005) arXiv:hep-th/0412141.

25. T. Sakai, S. Sugimoto, More on a holographic dual of QCD, Prog. Theor. Phys. 114, 1083 (2006) arXiv:hepth/0507073.

26. O. Aharony, A. Fayyazuddin, J.M. Maldacena, The large $N$ limit of $N=2,1$ field theories from three-branes in F-theory, JHEP 07, 013 (1998) arXiv:hep-th/9806159.

27. M. Graña, J. Polchinski, Gauge/gravity duals with holomorphic dilaton, Phys. Rev. D 65, 126005 (2002) arXiv: hep-th/0106014.

28. M. Bertolini, P. Di Vecchia, M. Frau, A. Lerda, R. Marotta, $N=2$ gauge theories on systems of fractional D3/D7 branes, Nucl. Phys. B 621, 157 (2002) arXiv:hepth/0107057.

29. B.A. Burrington, J.T. Liu, L.A. Pando Zayas, D. Vaman, Holographic duals of flavored $N=1$ super Yang-Mills: Beyond the probe approximation, JHEP 02, 022 (2005) arXiv:hep-th/0406207.

30. I. Kirsch, D. Vaman, The D3/Dr background and flavor dependence of Regge trajectories, Phys. Rev. D 72, 026007 (2005) arXiv:hep-th/0505164.

31. E. Witten, Anti-de Sitter space and holography, Adv. Theor. Math. Phys. 2, 253 (1998) arXiv:hep-th/9802150.

32. E. Witten, Anti-de Sitter space, thermal phase transition, and confinement in gauge theories, Adv. Theor. Math. Phys. 2, 505 (1998) arXiv:hep-th/9803131.

33. I. Kirsch, Generalizations of the AdS/CFT correspondence, Fortschr. Phys. 52, 727 (2004) arXiv:hep-th/ 0406274

34. D. Mateos, R.C. Myers, R.M. Thomson, Thermodynamics of the brane, JHEP 05, 067 (2007) arXiv:hep-th/0701132.

35. K. Ghoroku, T. Sakaguchi, N. Uekusa, M. Yahiro, Flavor quark at high temperature from a holographic model, Phys. Rev. D 71, 106002 (2005) arXiv:hep-th/0502088.

36. T. Albash, V. Filev, C.V. Johnson, A. Kundu, A topologychanging phase transition and the dynamics of flavour, arXiv:hep-th/0605088.

37. P.S. Howe, P.C. West, The complete $N=2, D=10$ supergravity, Nucl. Phys. B 238, 181 (1984).

38. J.H. Schwarz, P.C. West, symmetries and transformations of chiral $N=2, D=10$ supergravity, Phys. Lett. B 126, 301 (1983).

39. E. D'Hoker, D.Z. Freedman, Supersymmetric gauge theories and the AdS/CFT correspondence, arXiv:hepth/0201253.

40. G. 't Hooft, Dimensional reduction in quantum gravity, arXiv:gr-qc/9310026.

41. S.S. Gubser, I.R. Klebanov, A.M. Polyakov, Gauge theory correlators from non-critical string theory, Phys. Lett. B 428, 105 (1998) arXiv:hep-th/9802109.
42. D.Z. Freedman, S.D. Mathur, A. Matusis, L. Rastelli, Correlation functions in the $C F T(d) / A d S(d+1)$ correspondence, Nucl. Phys. B 546, 96 (1999) arXiv:hepth/9804058.

43. S. Lee, S. Minwalla, M. Rangamani, N. Seiberg, Threepoint functions of chiral operators in $D=4, N=4$ SYM at large N, Adv. Theor. Math. Phys. 2, 697 (1998) arXiv:hep-th/9806074.

44. M. Henningson, K. Skenderis, The holographic Weyl anomaly, JHEP 07, 023 (1998) arXiv:hep-th/9806087.

45. L. Girardello, M. Petrini, M. Porrati, A. Zaffaroni, Novel local CFT and exact results on perturbations of $N=4$ super Yang-Mills from AdS dynamics, JHEP 12, 022 (1998) arXiv:hep-th/9810126.

46. P. Kraus, F. Larsen, S.P. Trivedi, The Coulomb branch of gauge theory from rotating branes, JHEP 03, 003 (1999) arXiv:hep-th/9811120.

47. I. Bakas, K. Sfetsos, States and curves of five-dimensional gauged supergravity, Nucl. Phys. B 573, 768 (2000) arXiv:hep-th/9909041.

48. K. Skenderis, M. Taylor, Kaluza-Klein holography, JHEP 05, 057 (2006) arXiv:hep-th/0603016.

49. K. Skenderis, M. Taylor, Holographic Coulomb branch vevs, JHEP 08, 001 (2006) arXiv:hep-th/0604169.

50. K.A. Intriligator, Maximally supersymmetric $R G$ flows and AdS duality, Nucl. Phys. B 580, 99 (2000) arXiv:hepth/9909082.

51. A. Hashimoto, Holographic description of D3-branes in flat space, Phys. Rev. D 60, 127902 (1999) arXiv:hepth/9903227.

52. N.R. Constable, R.C. Myers, Exotic scalar states in the AdS/CFT correspondence, JHEP 11, 020 (1999) arXiv:hep-th/9905081.

53. D.Z. Freedman, S.S. Gubser, K. Pilch, N.P. Warner, Continuous distributions of D3-branes and gauged supergravity, JHEP 07, 038 (2000) arXiv:hep-th/9906194.

54. A. Khavaev, K. Pilch, N.P. Warner, New vacua of gauged $N=8$ supergravity in five dimensions, Phys. Lett. B 487, 14 (2000) arXiv:hep-th/9812035.

55. L. Girardello, M. Petrini, M. Porrati, A. Zaffaroni, Confinement and condensates without fine tuning in supergravity duals of gauge theories, JHEP 05, 026 (1999) arXiv:hep-th/9903026.

56. D.Z. Freedman, S.S. Gubser, K. Pilch, N.P. Warner, Renormalization group flows from holography supersymmetry and a c-theorem, Adv. Theor. Math. Phys. 3, 363 (1999) arXiv:hep-th/9904017.

57. N.J. Evans, M. Petrini, AdS RG-flow and the super-YangMills cascade, Nucl. Phys. B 592, 129 (2001) arXiv:hepth/0006048.

58. K. Pilch, N.P. Warner, $N=2$ supersymmetric $R G$ flows and the IIB dilaton, Nucl. Phys. B 594, 209 (2001) arXiv:hep-th/0004063.

59. K. Pilch, N.P. Warner, Generalizing the $N=2$ supersymmetric $R G$ flow solution of IIB supergravity, Nucl. Phys. B 675, 99 (2003) arXiv:hep-th/0306098.

60. A. Brandhuber, K. Sfetsos, An $N=2$ gauge theory and its supergravity dual, Phys. Lett. B 488, 373 (2000) arXiv:hep-th/0004148.

61. A. Buchel, A.W. Peet, J. Polchinski, Gauge dual and noncommutative extension of an $N=2$ supergravity solution, Phys. Rev. D 63, 044009 (2001) arXiv:hep-th/0008076. 
62. N.J. Evans, C.V. Johnson, M. Petrini, The enhancon and $N=2$ gauge theory/gravity $R G$ flows, JHEP 10, 022 (2000) arXiv:hep-th/0008081.

63. V. Balasubramanian, P. Kraus, Spacetime and the holographic renormalization group, Phys. Rev. Lett. 83, 3605 (1999) arXiv:hep-th/9903190.

64. J. Erdmenger, A field-theoretical interpretation of the holographic renormalization group, Phys. Rev. D 64, 085012 (2001) arXiv:hep-th/0103219.

65. L. Girardello, M. Petrini, M. Porrati, A. Zaffaroni, The supergravity dual of $N=1$ super Yang-Mills theory, Nucl. Phys. B 569, 451 (2000) arXiv:hep-th/9909047.

66. K. Pilch, N.P. Warner, $N=1$ supersymmetric renormalization group flows from IIB supergravity, Adv. Theor. Math. Phys. 4, 627 (2002) arXiv:hep-th/0006066.

67. J. Polchinski, M.J. Strassler, The string dual of a confining four-dimensional gauge theory, arXiv:hep-th/ 0003136.

68. J. Distler, F. Zamora, Non-supersymmetric conformal field theories from stable anti-de Sitter spaces, Adv. Theor. Math. Phys. 2, 1405 (1999) arXiv:hep-th/9810206.

69. S.S. Gubser, Dilaton-driven confinement, arXiv:hep-th/ 9902155 .

70. J. Babington, D.E. Crooks, N.J. Evans, A nonsupersymmetric deformation of the AdS/CFT correspondence, JHEP 02, 024 (2003) arXiv:hep-th/0207076.

71. J. Babington, D.E. Crooks, N.J. Evans, A stable supergravity dual of non-supersymmetric glue, Phys. Rev. D 67, 066007 (2003) arXiv:hep-th/0210068.

72. J.M. Maldacena, Wilson loops in large $N$ field theories, Phys. Rev. Lett. 80, 4859 (1998) arXiv:hep-th/9803002.

73. S.-J. Rey, J.-T. Yee, Macroscopic strings as heavy quarks in large $N$ gauge theory and anti-de Sitter supergravity, Eur. Phys. J. C 22, 379 (2001) arXiv:hep-th/9803001.

74. C. Csaki, H. Ooguri, Y. Oz, J. Terning, Glueball mass spectrum from supergravity, JHEP 01, 017 (1999) arXiv:hep-th/9806021.

75. S. Hong, S. Yoon, M.J. Strassler, Quarkonium from the fifth dimension, JHEP 04, 046 (2004) arXiv:hepth/0312071.

76. A. Karch, A. O'Bannon, K. Skenderis, Holographic renormalization of probe D-branes in AdS/CFT, JHEP 04, 015 (2006) arXiv:hep-th/0512125.

77. K. Skenderis, Lecture notes on holographic renormalization, Class. Quantum. Grav. 19, 5849 (2002) arXiv:hepth/0209067.

78. P. Breitenlohner, D.Z. Freedman, Positive energy in antiDe Sitter backgrounds and gauged extended supergravity, Phys. Lett. B 115, 197 (1982).

79. P. Breitenlohner, D.Z. Freedman, Stability in gauged extended supergravity, Ann. Phys. 144, 249 (1982).

80. D. Arean, I. Kirsch, A. Ramallo, unpublished notes (2007).

81. I. Kirsch, Spectroscopy of fermionic operators in AdS/CFT, JHEP 09, 052 (2006) arXiv:hep-th/0607205.

82. L. Martucci, J. Rosseel, D. Van den Bleeken, A. Van Proeyen, Dirac actions for D-branes on backgrounds with fluxes, Class. Quantum. Grav. 22, 2745 (2005) arXiv:hep-th/0504041.

83. S. Hong, S. Yoon, M.J. Strassler, On the couplings of vector mesons in AdS/QCD, JHEP 04, 003 (2006) arXiv:hep-th/0409118.
84. S. Hong, S. Yoon, M.J. Strassler, Adjoint trapping: A new phenomenon at strong 't Hooft coupling, JHEP 03, 012 (2006) arXiv:hep-th/0410080.

85. J.P. Shock, Canonical coordinates and meson spectra for scalar deformed $N=4 S Y M$ from the AdS/CFT correspondence, JHEP 10, 043 (2006) arXiv:hep-th/0601025.

86. J. Erdmenger, N. Evans, J. Große, Heavy-light mesons from the AdS/CFT correspondence, JHEP 01, 098 (2007) arXiv:hep-th/0605241.

87. A. Karch, E. Katz, N. Weiner, Hadron masses and screening from AdS Wilson loops, Phys. Rev. Lett. 90, 091601 (2003) arXiv:hep-th/0211107.

88. R.C. Myers, Dielectric-branes, JHEP 12, 022 (1999) arXiv:hep-th/9910053.

89. J. Erdmenger, K. Ghoroku, I. Kirsch, Holographic heavylight mesons from non-Abelian DBI, JHEP 09, 111 (2007) arXiv:0706.3978 [hep-th].

90. H. Liu, A.A. Tseytlin, D3-brane D-instanton configuration and $N=4$ super $Y M$ theory in constant self-dual background, Nucl. Phys. B 553, 231 (1999) arXiv:hepth/9903091.

91. K. Ghoroku, M. Yahiro, Chiral symmetry breaking driven by dilaton, Phys. Lett. B 604, 235 (2004) arXiv:hepth/0408040.

92. S.S. Gubser, I.R. Klebanov, A.M. Polyakov, A semiclassical limit of the gauge/string correspondence, Nucl. Phys. B 636, 99 (2002) arXiv:hep-th/0204051.

93. A. Paredes, P. Talavera, Multiflavour excited mesons from the fifth dimension, Nucl. Phys. B 713, 438 (2005) arXiv:hep-th/0412260.

94. A.L. Cotrone, L. Martucci, W. Troost, String splitting and strong coupling meson decay, Phys. Rev. Lett. 96, 141601 (2006) arXiv:hep-th/0511045.

95. F. Bigazzi, A.L. Cotrone, New predictions on meson decays from string splitting, JHEP 11, 066 (2006) arXiv:hep-th/0606059.

96. Z. Guralnik, S. Kovacs, B. Kulik, Holography and the Higgs branch of $N=2 S Y M$ theories, JHEP 03, 063 (2005) arXiv:hep-th/0405127.

97. Z. Guralnik, Strong coupling dynamics of the Higgs branch: Rolling a Higgs by collapsing an instanton, Nucl. Phys. B 732, 46 (2006) arXiv:hep-th/0412074.

98. Z. Guralnik, S. Kovacs, B. Kulik, AdS/CFT duality and the Higgs branch of $N=2 S Y M$, Fortschr. Phys. 53, 480 (2005) arXiv:hep-th/0501154.

99. J. Erdmenger, J. Große, Z. Guralnik, Spectral flow on the Higgs branch and AdS/CFT duality, JHEP 06, 052 (2005) arXiv:hep-th/0502224.

100. D. Arean, A.V. Ramallo, D. Rodriguez-Gomez, Holographic flavor on the Higgs branch, JHEP 05, 044 (2007) arXiv:hep-th/0703094.

101. R. Apreda, J. Erdmenger, N. Evans, Z. Guralnik, Strong coupling effective Higgs potential and a first order thermal phase transition from AdS/CFT duality, Phys. Rev. D 71, 126002 (2005) arXiv:hep-th/0504151.

102. R. Apreda, J. Erdmenger, N. Evans, Scalar effective potential for D' brane probes which break chiral symmetry, JHEP 05, 011 (2006) arXiv:hep-th/0509219.

103. R. Harnik, D.T. Larson, H. Murayama, Supersymmetric color superconductivity, JHEP 03, 049 (2004) arXiv:hep$\mathrm{ph} / 0309224$.

104. B.R. Greene, A.D. Shapere, C. Vafa, S.-T. Yau, Stringy cosmic strings and noncompact Calabi-Yau manifolds, Nucl. Phys. B 337, 1 (1990). 
105. R.G. Leigh, M. Rozali, Brane boxes, anomalies, bending and tadpoles, Phys. Rev. D 59, 026004 (1999) arXiv:hepth/9807082.

106. I.R. Klebanov, E. Witten, Superconformal field theory on threebranes at a Calabi-Yau singularity, Nucl. Phys. B 536, 199 (1998) arXiv:hep-th/9807080.

107. I.R. Klebanov, N.A. Nekrasov, Gravity duals of fractional branes and logarithmic RG flow, Nucl. Phys. B 574, 263 (2000) arXiv:hep-th/9911096.

108. I.R. Klebanov, A.A. Tseytlin, Gravity duals of supersymmetric $S U(N) \times S U(N+M)$ gauge theories, Nucl. Phys. B 578, 123 (2000) arXiv:hep-th/0002159.

109. I.R. Klebanov, E. Witten, AdS/CFT correspondence and symmetry breaking, Nucl. Phys. B 556, 89 (1999) arXiv:hep-th/9905104.

110. I.R. Klebanov, M.J. Strassler, Supergravity and a confining gauge theory: Duality cascades and chiSB-resolution of naked singularities, JHEP 08, 052 (2000) arXiv:hepth/0007191.

111. T. Sakai, J. Sonnenschein, Probing flavored mesons of confining gauge theories by supergravity, JHEP 09, 047 (2003) arXiv:hep-th/0305049.

112. S. Kuperstein, Meson spectroscopy from holomorphic probes on the warped deformed conifold, JHEP 03, 014 (2005) arXiv:hep-th/0411097.

113. T.S. Levi, P. Ouyang, Mesons and Flavor on the Conifold, arXiv:hep-th/0506021.

114. D. Arean, D.E. Crooks, A.V. Ramallo, Supersymmetric probes on the conifold, JHEP 11, 035 (2004) arXiv:hepth/0408210.

115. P. Ouyang, Holomorphic D7 branes and flavored $N=1$ gauge theories, Nucl. Phys. B 699, 207 (2004) arXiv:hepth/0311084.

116. K.A. Intriligator, N. Seiberg, Lectures on supersymmetric gauge theories and electric- magnetic duality, Nucl. Phys. Proc. Suppl. 45BC, 1 (1996) arXiv:hep-th/9509066.

117. F. Benini, F. Canoura, S. Cremonesi, C. Nuñez, A.V. Ramallo, Unquenched flavors in the Klebanov-Witten model, JHEP 02, 090 (2007) arXiv:hep-th/0612118.

118. F. Benini, F. Canoura, S. Cremonesi, C. Nuñez, A.V. Ramallo, Backreacting flavors in the Klebanov-Strassler background, JHEP 09, 109 (2007) arXiv:0706.1238 [hepth].

119. F. Canoura, J.D. Edelstein, A.V. Ramallo, D-brane probes on $L(a, b, c)$ superconformal field theories, JHEP 09, 038 (2006) arXiv:hep-th/0605260.

120. R. Apreda, J. Erdmenger, D. Lüst, C. Sieg, Adding flavour to the Polchinski-Strassler background, JHEP 01, 079 (2007) arXiv:hep-th/0610276.

121. C. Sieg, Holographic flavour in the $N=1$ Polchinski-Strassler background, JHEP 08, 031 (2007) arXiv:0704.3544 [hep-th].

122. S. Penati, M. Pirrone, C. Ratti, Mesons in marginally deformed AdS/CFT, arXiv:0710.4292 [hep-th].

123. O. Lunin, J.M. Maldacena, Deforming field theories with $U(1) \times U(1)$ global symmetry and their gravity duals, JHEP 05, 033 (2005) arXiv:hep-th/0502086.

124. J.M. Maldacena, C. Nuñez, Towards the large $N$ limit of pure $N=1$ super Yang Mills, Phys. Rev. Lett. 86, 588 (2001) arXiv:hep-th/0008001.

125. A.H. Chamseddine, M.S. Volkov, Non-Abelian solitons in $N=4$ gauged supergravity and leading order string theory, Phys. Rev. D 57, 6242 (1998) arXiv:hep-th/9711181.
126. X.-J. Wang, S. Hu, Intersecting branes and adding flavors to the Maldacena- Nuñez background, JHEP 09, 017 (2003) arXiv:hep-th/0307218.

127. C. Nuñez, A. Paredes, A.V. Ramallo, Flavoring the gravity dual of $N=1$ Yang-Mills with probes, JHEP 12, 024 (2003) arXiv:hep-th/0311201.

128. R. Casero, C. Nuñez, A. Paredes, Towards the string dual of $N=1$ SQCD-like theories, Phys. Rev. D 73, 086005 (2006) arXiv:hep-th/0602027.

129. A. Paredes, On unquenched $N=2$ holographic flavor, JHEP 12, 032 (2006) arXiv:hep-th/0610270.

130. A. Karch, L. Randall, Open and closed string interpretation of SUSY CFT's on branes with boundaries, JHEP 06, 063 (2001) arXiv:hep-th/0105132.

131. O. DeWolfe, D.Z. Freedman, H. Ooguri, Holography and defect conformal field theories, Phys. Rev. D 66, 025009 (2002) arXiv:hep-th/0111135.

132. J. Erdmenger, Z. Guralnik, I. Kirsch, Four-dimensional superconformal theories with interacting boundaries or defects, Phys. Rev. D 66, 025020 (2002) arXiv:hepth/0203020.

133. K. Skenderis, M. Taylor, Branes in AdS and pp-wave spacetimes, JHEP 06, 025 (2002) arXiv:hep-th/0204054.

134. N.R. Constable, J. Erdmenger, Z. Guralnik, I. Kirsch, Intersecting D3 branes and holography, Phys. Rev. D 68, 106007 (2003) arXiv:hep-th/0211222.

135. D. Arean, A.V. Ramallo, Open string modes at brane intersections, JHEP 04, 037 (2006) arXiv:hep-th/0602174.

136. D. Arean, A.V. Ramallo, D. Rodriguez-Gomez, Mesons and Higgs branch in defect theories, Phys. Lett. B 641, 393 (2006) arXiv:hep-th/0609010.

137. S. Yamaguchi, AdS branes corresponding to superconformal defects, JHEP 06, 002 (2003) arXiv:hep-th/0305007.

138. A. Basu, A. Maharana, Generalized Gross-Neveu models and chiral symmetry breaking from string theory, Phys. Rev. D 75, 065005 (2007) arXiv:hep-th/0610087.

139. F. Canoura, A. Paredes, A.V. Ramallo, Supersymmetric defects in the Maldacena-Nuñez background, JHEP 09, 032 (2005) arXiv:hep-th/0507155.

140. R.C. Myers, R.M. Thomson, Holographic mesons in various dimensions, JHEP 09, 066 (2006) arXiv:hepth/0605017.

141. S.A. Cherkis, A. Hashimoto, Supergravity solution of intersecting branes and AdS/CFT with flavor, JHEP 11, 036 (2002) arXiv:hep-th/0210105.

142. J. Erdmenger, I. Kirsch, Mesons in gauge/gravity dual with large number of fundamental fields, JHEP 12, 025 (2004) arXiv:hep-th/0408113.

143. D. Arean, A. Paredes, A.V. Ramallo, Adding flavor to the gravity dual of non-commutative gauge theories, JHEP 08, 017 (2005) arXiv:hep-th/0505181.

144. E. Witten, Current Algebra Theorems for the U(1) Goldstone Boson, Nucl. Phys. B 156, 269 (1979).

145. G. 't Hooft, How Instantons Solve the U(1) Problem, Phys. Rep. 142, 357 (1986).

146. H. Georgi, Weak Interactions and Modern Particle Theory, (Benjamin/Cummings, Menlo Park, 1984) p. 165.

147. I. Brevik, K. Ghoroku, A. Nakamura, Meson mass and confinement force driven by dilaton, Int. J. Mod. Phys. D 15, 57 (2006) arXiv:hep-th/0505057.

148. J. Große, Quantum Field Theories Coupled to Supergravity: AdS/CFT and Local Couplings, PhD Thesis, Ludwig-Maximilians-Universität München (2006). urn:nbn: de:bvb:19-67614. 
149. N.J. Evans, J.P. Shock, Chiral dynamics from AdS space, Phys. Rev. D 70, 046002 (2004) arXiv:hep-th/0403279.

150. N. Evans, J.P. Shock, T. Waterson, Dr7 brane embeddings and chiral symmetry breaking, JHEP 03, 005 (2005) arXiv:hep-th/0502091.

151. J.L.F. Barbon, C. Hoyos, D. Mateos, R.C. Myers, The holographic life of the eta', JHEP 10, 029 (2004) arXiv:hep-th/0404260.

152. A. Armoni, Witten-Veneziano from Green-Schwarz, JHEP 06, 019 (2004) arXiv:hep-th/0404248.

153. M. Gell-Mann, R.J. Oakes, B. Renner, Behavior of current divergences under $S U(3) \times S U(3)$, Phys. Rev. 175, 2195 (1968).

154. K. Ghoroku, M. Ishihara, A. Nakamura, Gauge theory in de Sitter space-time from a holographic model, Phys. Rev. D 74, 124020 (2006) arXiv:hep-th/0609152.

155. T. Hirayama, A holographic dual of CFT with flavor on de Sitter space, JHEP 06, 013 (2006) arXiv:hep-th/0602258.

156. M. Kruczenski, L.A.P. Zayas, J. Sonnenschein, D. Vaman, Regge trajectories for mesons in the holographic dual of large- $N(c)$ QCD, JHEP 06, 046 (2005) arXiv:hepth/0410035.

157. K. Peeters, J. Sonnenschein, M. Zamaklar, Holographic decays of large-spin mesons, JHEP 02, 009 (2006) arXiv: hep-th/0511044.

158. M. Bando, A. Sugamoto, S. Terunuma, Meson strings and flavor branes, Prog. Theor. Phys. 115, 1111 (2006) arXiv:hep-ph/0602203.

159. C. Vafa, E. Witten, Parity Conservation in QCD, Phys. Rev. Lett. 53, 535 (1984).

160. A. Basu, Higher Derivative Corrections in Holographic $Q C D$, arXiv:0707.0081 [hep-th].

161. B.A. Burrington, V.S. Kaplunovsky, J. Sonnenschein, Localized Backreacted Flavor Branes in Holographic QCD, arXiv:0708.1234 [hep-th].

162. Y.-h. Gao, J.P. Shock, W.-s. Xu, D.-f. Zeng, A note on chiral symmetry breaking from intersecting branes, Phys. Rev. D 76, 046003 (2007) arXiv:0704.3913 [hep-th].

163. M. Harada, S. Matsuzaki, K. Yamawaki, Implications of holographic QCD in ChPT with hidden local symmetry, Phys. Rev. D 74, 076004 (2006) arXiv:hep-ph/0603248.

164. R. Heise, H.G. Svendsen, A note on fermions in holographic QCD, JHEP 08, 065 (2007) arXiv:0706.2253 [hepth].

165. K. Nawa, H. Suganuma, T. Kojo, Baryons in Holographic QCD, Phys. Rev. D 75, 086003 (2007) arXiv:hepth/0612187.

166. H. Hata, T. Sakai, S. Sugimoto, S. Yamato, Baryons from instantons in holographic QCD, arXiv:hep-th/0701280.

167. K. Nawa, H. Suganuma, T. Kojo, Brane-induced Skyrmions: Baryons in holographic QCD, arXiv:hep-th/ 0701007 .

168. D.K. Hong, M. Rho, H.-U. Yee, P. Yi, Chiral dynamics of baryons from string theory, Phys. Rev. D 76, 061901 (2007) arXiv:hep-th/0701276.

169. D.K. Hong, H.-C. Kim, S. Siwach, H.-U. Yee, The Electric Dipole Moment of the Nucleons in Holographic QCD, arXiv:0709.0314 [hep-ph].

170. D.K. Hong, M. Rho, H.-U. Yee, P. Yi, Dynamics of baryons from string theory and vector Dominance, JHEP 09, 063 (2007) arXiv:0705.2632 [hep-th].

171. S.K. Domokos, J.A. Harvey, Baryon number-induced Chern-Simons couplings of vector and axial-vector mesons in holographic QCD, arXiv:0704.1604 [hep-ph].
172. A. Imaanpur, On instantons in holographic $Q C D$, arXiv: 0705.0496 [hep-th].

173. Y. Kim, C.-H. Lee, H.-U. Yee, Holographic Nuclear Matter in $A d S / Q C D$, arXiv:0707.2637 [hep-ph].

174. M. Rozali, H.-H. Shieh, M. Van Raamsdonk, J. Wu, Cold Nuclear Matter in Holographic QCD, arXiv:0708.1322 [hep-th].

175. N. Evans, E. Threlfall, Quark Mass in the Sakai-Sugimoto Model of Chiral Symmetry Breaking, arXiv:0706.3285 [hep-th].

176. E. Antonyan, J.A. Harvey, S. Jensen, D. Kutasov, NJL and QCD from string theory, arXiv:hep-th/0604017.

177. E. Antonyan, J.A. Harvey, D. Kutasov, Chiral symmetry breaking from intersecting D-branes, Nucl. Phys. B 784, 1 (2007) arXiv:hep-th/0608177.

178. R. Casero, E. Kiritsis, A. Paredes, Chiral symmetry breaking as open string tachyon condensation, arXiv:hepth/0702155.

179. A. Dhar, P. Nag, Sakai-Sugimoto model, tachyon condensation and chiral symmetry breaking, arXiv:0708.3233 [hep-th].

180. O. Bergman, S. Seki, J. Sonnenschein, Quark mass and condensate in $H Q C D$, arXiv:0708.2839 [hep-th].

181. K. Hashimoto, T. Hirayama, A. Miwa, Holographic QCD and pion mass, JHEP 06, 020 (2007) arXiv:hepth/0703024.

182. S. Kuperstein, J. Sonnenschein, Non-critical supergravity $(d>1)$ and holography, JHEP 07, 049 (2004) arXiv:hepth/0403254.

183. R. Casero, A. Paredes, J. Sonnenschein, Fundamental matter, meson spectroscopy and non-critical string/gauge duality, JHEP 01, 127 (2006) arXiv:hep-th/0510110.

184. A.L. Cotrone, $O n$ the $Y M$ and $Q C D$ spectra from five dimensional strings, arXiv:0707.1483 [hep-th].

185. S.W. Hawking, D.N. Page, Thermodynamics of black holes in anti-De Sitter space, Commun. Math. Phys. 87, 577 (1983).

186. G. Policastro, D.T. Son, A.O. Starinets, The shear viscosity of strongly coupled $N=4$ supersymmetric Yang-Mills plasma, Phys. Rev. Lett. 87, 081601 (2001) arXiv:hepth/0104066.

187. E.V. Shuryak, Strongly coupled quark-gluon plasma: The status report, arXiv:hep-ph/0608177.

188. Z. Fodor, QCD Thermodynamics, arXiv:0711.0336 [heplat].

189. F. Karsch, Recent lattice results on finite temperature and density QCD, part I, arXiv:0711.0656 [hep-lat].

190. F. Karsch, Recent lattice results on finite temerature and density QCD, part II, arXiv:0711.0661 [hep-lat].

191. V.P. Frolov, A.L. Larsen, M. Christensen, Domain wall interacting with a black hole: A new example of critical phenomena, Phys. Rev. D 59, 125008 (1999) arXiv:hepth/9811148.

192. C. Hoyos, K. Landsteiner, S. Montero, Holographic meson melting, arXiv:hep-th/0612169.

193. E.W. Leaver, An Analytic representation for the quasi normal modes of Kerr black holes, Proc. R. Soc. London, Ser. A 402, 285 (1985).

194. R.C. Myers, A.O. Starinets, R.M. Thomson, Holographic spectral functions and diffusion constants for fundamental matter, arXiv:0706.0162 [hep-th].

195. K. Peeters, J. Sonnenschein, M. Zamaklar, Holographic melting and related properties of mesons in a quark gluon 
plasma, Phys. Rev. D 74, 106008 (2006) arXiv:hepth/0606195.

196. S.S. Gubser, Drag force in AdS/CFT, Phys. Rev. D 74, 126005 (2006) arXiv:hep-th/0605182.

197. C.P. Herzog, A. Karch, P. Kovtun, C. Kozcaz, L.G. Yaffe, Energy loss of a heavy quark moving through $N=4$ supersymmetric Yang-Mills plasma, JHEP 07, 013 (2006) arXiv:hep-th/0605158.

198. P.M. Chesler, L.G. Yaffe, The wake of a quark moving through a strongly-coupled $\mathcal{N}=4$ supersymmetric Yang-Mills plasma, Phys. Rev. Lett. 99, 152001 (2007) arXiv:0706.0368 [hep-th].

199. S.S. Gubser, S.S. Pufu, A. Yarom, Shock waves from heavy-quark mesons in AdS/CFT, arXiv:0711.1415 [hepth].

200. A. Karch, A. O'Bannon, Chiral transition of $N=4$ super Yang-Mills with flavor on a 3-sphere, Phys. Rev. D 74, 085033 (2006) arXiv:hep-th/0605120.

201. J. Große, R.A. Janik, P. Surowka, Flavors in an expanding plasma, arXiv:0709.3910 [hep-th].

202. S. Kobayashi, D. Mateos, S. Matsuura, R.C. Myers, R.M. Thomson, Holographic phase transitions at finite baryon density, JHEP 02, 016 (2007) arXiv:hep-th/0611099.

203. T. Albash, V. Filev, C.V. Johnson, A. Kundu, Global currents, phase transitions, and chiral symmetry breaking in large $N(c)$ gauge theory, arXiv:hep-th/0605175.

204. S. Nakamura, Y. Seo, S.-J. Sin, K.P. Yogendran, A new phase at finite quark density from AdS/CFT, arXiv:hepth/0611021.

205. D. Mateos, S. Matsuura, R.C. Myers, R.M. Thomson, Holographic phase transitions at finite chemical potential, arXiv:0709.1225 [hep-th].

206. O. Bergman, G. Lifschytz, M. Lippert, Holographic Nuclear Physics, arXiv:0708.0326 [hep-th].

207. A. Karch, A. O'Bannon, Holographic Thermodynamics at Finite Baryon Density: Some Exact Results, arXiv: 0709.0570 [hep-th].

208. K. Ghoroku, M. Ishihara, A. Nakamura, D3/D7 holographic Gauge theory and Chemical potential, arXiv: 0708.3706 [hep-th].

209. J. Erdmenger, M. Kaminski, F. Rust, Isospin diffusion in thermal AdS/CFT with flavor, Phys. Rev. D 76, 046001 (2007) arXiv:0704.1290 [hep-th].

210. J. Erdmenger, M. Kaminski, F. Rust, Holographic vector mesons from spectral functions at finite baryon or isospin density, arXiv:0710.0334 [hep-th].

211. O. Aharony, J. Sonnenschein, S. Yankielowicz, A holographic model of deconfinement and chiral symmetry restoration, Ann. Phys. 322, 1420 (2007) arXiv:hep-th/ 0604161.

212. N. Horigome, Y. Tanii, Holographic chiral phase transition with chemical potential, JHEP 01, 072 (2007) arXiv:hep-th/0608198.

213. D. Yamada, Sakai-Sugimoto Model at High Density, arXiv:0707.0101 [hep-th].

214. J.L. Davis, M. Gutperle, P. Kraus, I. Sachs, Stringy NJL and Gross-Neveu models at finite density and temperature, arXiv:0708.0589 [hep-th].

215. K.-Y. Kim, S.-J. Sin, I. Zahed, The Chiral Model of SakaiSugimoto at Finite Baryon Density, arXiv:0708.1469 [hep-th].

216. A. Parnachev, Holographic QCD with Isospin Chemical Potential, arXiv:0708.3170 [hep-th].
217. O. Aharony, K. Peeters, J. Sonnenschein, M. Zamaklar, Rho meson condensation at finite isospin chemical potential in a holographic model for QCD, arXiv:0709.3948 [hep-th].

218. V.G. Filev, Criticality, Scaling and Chiral Symmetry Breaking in External Magnetic Field, arXiv:0706.3811 [hep-th].

219. J. Erdmenger, R. Meyer, J.P. Shock, AdS/CFT with Flavour in Electric and Magnetic Kalb-Ramond Fields, arXiv:0709.1551 [hep-th].

220. T. Albash, V. Filev, C.V. Johnson, A. Kundu, Finite Temperature Large $N$ Gauge Theory with Quarks in an External Magnetic Field, arXiv:0709.1547 [hep-th].

221. T. Albash, V. Filev, C.V. Johnson, A. Kundu, Quarks in an External Electric Field in Finite Temperature Large $N$ Gauge Theory, arXiv:0709.1554 [hep-th].

222. J. Erlich, E. Katz, D.T. Son, M.A. Stephanov, $Q C D$ and a holographic model of hadrons, Phys. Rev. Lett. 95, 261602 (2005) arXiv:hep-ph/0501128.

223. L. Da Rold, A. Pomarol, Chiral symmetry breaking from five dimensional spaces, Nucl. Phys. B 721, 79 (2005) arXiv:hep-ph/0501218.

224. L. Da Rold, A. Pomarol, The scalar and pseudoscalar sector in a five-dimensional approach to chiral symmetry breaking, JHEP 01, 157 (2006) arXiv:hep-ph/0510268.

225. J. Hirn, V. Sanz, Interpolating between low and high energy QCD via a 5D Yang-Mills model, JHEP 12, 030 (2005) arXiv:hep-ph/0507049.

226. M.A. Shifman, A.I. Vainshtein, V.I. Zakharov, $Q C D$ and Resonance Physics. Sum Rules, Nucl. Phys. B 147, 385 (1979).

227. H. Boschi-Filho, N.R.F. Braga, QCD/string holographic mapping and glueball mass spectrum, Eur. Phys. J. C 32, 529 (2004) arXiv:hep-th/0209080.

228. H. Boschi-Filho, N.R.F. Braga, Gauge/string duality and scalar glueball mass ratios, JHEP 05, 009 (2003) arXiv:hep-th/0212207.

229. H. Boschi-Filho, N.R.F. Braga, H.L. Carrion, Glueball Regge trajectories from gauge/string duality and the pomeron, Phys. Rev. D 73, 047901 (2006) arXiv:hepth/0507063.

230. C.J. Morningstar, M.J. Peardon, Efficient glueball simulations on anisotropic lattices, Phys. Rev. D 56, 4043 (1997) arXiv:hep-lat/9704011.

231. C.J. Morningstar, M.J. Peardon, The Glueball spectrum from an anisotropic lattice study, Phys. Rev. D 60, 034509 (1999) arXiv:hep-lat/9901004.

232. J.P. Shock, F. Wu, Three flavour QCD from the holographic principle, JHEP 08, 023 (2006) arXiv:hep$\mathrm{ph} / 0603142$.

233. E. Katz, A. Lewandowski, M.D. Schwartz, Tensor mesons in AdS/QCD, Phys. Rev. D 74, 086004 (2006) arXiv:hep$\mathrm{ph} / 0510388$.

234. G.F. de Teramond, S.J. Brodsky, The hadronic spectrum of a holographic dual of QCD, Phys. Rev. Lett. 94, 201601 (2005) arXiv:hep-th/0501022.

235. T. Hambye, B. Hassanain, J. March-Russell, M. Schvellinger, On the $\Delta(I)=1 / 2$ rule in holographic $Q C D$, Phys. Rev. D 74, 026003 (2006) arXiv:hep-ph/0512089.

236. C.D. White, The Cornell potential from general geometries in AdS/QCD, Phys. Lett. B 652, 79 (2007) arXiv:hep-ph/0701157. 
237. S.J. Brodsky, G.F. de Teramond, Hadronic spectra and light-front wavefunctions in holographic QCD, Phys. Rev. Lett. 96, 201601 (2006) arXiv:hep-ph/0602252.

238. S.J. Brodsky, G.F. de Teramond, Light-Front Dynamics and AdS/QCD: The Pion Form Factor in the Space- and Time-Like Regions, arXiv:0707.3859 [hep-ph].

239. H.R. Grigoryan, A.V. Radyushkin, Form Factors and Wave Functions of Vector Mesons in Holographic QCD, Phys. Lett. B 650, 421 (2007) arXiv:hep-ph/0703069.

240. H.R. Grigoryan, A.V. Radyushkin, Pion Form Factor in Chiral Limit of Hard-Wall AdS/QCD Model, arXiv:0709.0500 [hep-ph].

241. K. Ghoroku, M. Yahiro, Holographic model for mesons at finite temperature, Phys. Rev. D 73, 125010 (2006) arXiv:hep-ph/0512289.

242. K. Ghoroku, A. Nakamura, M. Yahiro, Holographic model at finite temperature with $R$-charge density, Phys. Lett. B 638, 382 (2006) arXiv:hep-ph/0605026.

243. E. Nakano, S. Teraguchi, W.-Y. Wen, Drag Force, Jet Quenching, and AdS/QCD, Phys. Rev. D 75, 085016 (2007) arXiv:hep-ph/0608274.

244. K. Kajantie, T. Tahkokallio, J.-T. Yee, Thermodynamics of $A d S / Q C D$, JHEP 01, 019 (2007) arXiv:hep$\mathrm{ph} / 0609254$.

245. Y. Kim, S.-J. Sin, K.H. Jo, H.K. Lee, Vector Susceptibility and Chiral Phase Transition in AdS/QCD Models, arXiv:hep-ph/0609008.

246. R.-G. Cai, J.P. Shock, Holographic confinement/deconfinement phase transitions of AdS/QCD in curved spaces, JHEP 08, 095 (2007) arXiv:0705.3388 [hep-th].

247. K.-I. Kim, Y. Kim, S.H. Lee, Isospin matter in AdS/ $Q C D$, arXiv:0709.1772 [hep-ph].

248. B. Holdom, Raising the sideways scale, Phys. Rev. D 24, 1441 (1981).

249. S. Weinberg, Implications of Dynamical Symmetry Breaking: An Addendum, Phys. Rev. D 19, 1277 (1979).

250. L. Susskind, Dynamics of spontaneous symmetry breaking in the Weinberg-Salam theory, Phys. Rev. D 20, 2619 (1979).

251. D.K. Hong, H.-U. Yee, Holographic estimate of oblique corrections for technicolor, Phys. Rev. D 74, 015011 (2006) arXiv:hep-ph/0602177.

252. J. Hirn, V. Sanz, A negative $S$ parameter from holographic technicolor, Phys. Rev. Lett. 97, 121803 (2006) arXiv:hep-ph/0606086.

253. M. Piai, Precision electro-weak parameters from $A d S(5)$, localized kinetic terms and anomalous dimensions, arXiv: hep-ph/0608241.

254. C.D. Carone, J. Erlich, J.A. Tan, Holographic Bosonic Technicolor, Phys. Rev. D 75, 075005 (2007) arXiv:hep$\mathrm{ph} / 0612242$.

255. C.D. Carone, J. Erlich, M. Sher, Holographic electroweak symmetry breaking from D-branes, Phys. Rev. D 76, 015015 (2007) arXiv:0704.3084 [hep-th].

256. C. Csaki, C. Grojean, H. Murayama, L. Pilo, J. Terning, Gauge theories on an interval: Unitarity without a Higgs, Phys. Rev. D 69, 055006 (2004) arXiv:hep-ph/0305237.

257. C. Csaki, C. Grojean, L. Pilo, J. Terning, Towards a realistic model of Higgsless electroweak symmetry breaking, Phys. Rev. Lett. 92, 101802 (2004) arXiv:hep-ph/ 0308038 .

258. C.T. Hill, S. Pokorski, J. Wang, Gauge invariant effective Lagrangian for Kaluza-Klein modes, Phys. Rev. D 64, 105005 (2001) arXiv:hep-th/0104035.
259. N. Arkani-Hamed, A.G. Cohen, H. Georgi, (De)constructing dimensions, Phys. Rev. Lett. 86, 4757 (2001) arXiv:hep-th/0104005.

260. R. Foadi, S. Gopalakrishna, C. Schmidt, Higgsless electroweak symmetry breaking from theory space, JHEP 03, 042 (2004) arXiv:hep-ph/0312324.

261. O. Cata, Towards understanding Regge trajectories in holographic QCD, Phys. Rev. D 75, 106004 (2007) arXiv:hep-ph/0605251.

262. P. Masjuan, S. Peris, A rational approach to resonance saturation in large-Nc QCD, JHEP 05, 040 (2007) arXiv:0704.1247 [hep-ph].

263. M. Shifman, A. Vainshtein, Highly Excited Mesons, Linear Regge Trajectories and the Pattern of the Chiral Symmetry Realization, arXiv:0710.0863 [hep-ph].

264. M. Huang, Q.-S. Yan, Y. Yang, Confront Holographic QCD with Regge Trajectories, arXiv:0710.0988 [hep-ph].

265. A. Karch, E. Katz, D.T. Son, M.A. Stephanov, Linear confinement and $A d S / Q C D$, Phys. Rev. D 74, 015005 (2006) arXiv:hep-ph/0602229.

266. N. Evans, J.P. Shock, T. Waterson, Towards a perfect QCD gravity dual, Phys. Lett. B 622, 165 (2005) arXiv:hep-th/0505250.

267. E. Shuryak, A "Domain wall" Scenario for the AdS/ $Q C D$, arXiv:0711.0004 [hep-ph].

268. K. Ghoroku, N. Maru, M. Tachibana, M. Yahiro, Holographic model for hadrons in deformed AdS(5) background, Phys. Lett. B 633, 602 (2006) arXiv:hepph/0510334.

269. C. Csaki, M. Reece, Toward a systematic holographic QCD: A braneless approach, JHEP 05, 062 (2007) arXiv:hep-ph/0608266.

270. J. Hirn, N. Rius, V. Sanz, Geometric approach to condensates in holographic QCD, Phys. Rev. D 73, 085005 (2006) arXiv:hep-ph/0512240.

271. J.P. Shock, F. Wu, Y.-L. Wu, Z.-F. Xie, AdS/QCD phenomenological models from a back-reacted geometry, JHEP 03, 064 (2007) arXiv:hep-ph/0611227.

272. U. Gürsoy, E. Kiritsis, Exploring improved holographic theories for QCD: Part I, arXiv:0707.1324 [hep-th].

273. U. Gürsoy, E. Kiritsis, F. Nitti, Exploring improved holographic theories for QCD: Part II, arXiv:0707.1349 [hepth].

274. N. Evans, A. Tedder, Perfecting the ultra-violet of holographic descriptions of QCD, Phys. Lett. B 642, 546 (2006) arXiv:hep-ph/0609112.

275. N. Evans, A. Tedder, T. Waterson, Improving the infrared of holographic descriptions of QCD, JHEP 01, 058 (2007) arXiv:hep-ph/0603249.

276. H.R. Grigoryan, Dimension Six Correction to the Vector Sector of AdS/QCD Model, arXiv:0709.0939 [hep-ph].

277. O. Aharony, S.S. Gubser, J.M. Maldacena, H. Ooguri, Y. Oz, Large $N$ field theories, string theory and gravity, Phys. Rep. 323, 183 (2000) arXiv:hep-th/9905111.

278. O. Aharony, The non-AdS/non-CFT correspondence, or three different paths to QCD, arXiv:hep-th/0212193.

279. D. Rodriguez-Gomez, Holographic flavor in theories with eight supercharges, Int. J. Mod. Phys. A 22, 4717 (2007) arXiv:0710.4471 [hep-th].

280. K. Peeters, M. Zamaklar, The string/gauge theory correspondence in $Q C D$, arXiv:0708.1502 [hep-ph].

281. D. Mateos, String Theory and Quantum Chromodynamics, arXiv:0709.1523 [hep-th]. 